\title{
Precise Point Positioning Inertial Navigation Integration for Kinematic Airborne Applications
}

\author{
Ryan Watson
}

Follow this and additional works at: https://researchrepository.wvu.edu/etd

\section{Recommended Citation}

Watson, Ryan, "Precise Point Positioning Inertial Navigation Integration for Kinematic Airborne Applications" (2017). Graduate Theses, Dissertations, and Problem Reports. 6925.

https://researchrepository.wvu.edu/etd/6925

This Thesis is protected by copyright and/or related rights. It has been brought to you by the The Research Repository @ WVU with permission from the rights-holder(s). You are free to use this Thesis in any way that is permitted by the copyright and related rights legislation that applies to your use. For other uses you must obtain permission from the rights-holder(s) directly, unless additional rights are indicated by a Creative Commons license in the record and/ or on the work itself. This Thesis has been accepted for inclusion in WVU Graduate Theses, Dissertations, and Problem Reports collection by an authorized administrator of The Research Repository @ WVU. For more information, please contact researchrepository@mail.wvu.edu. 


\title{
PRECISE POINT POSITIONING INERTIAL NAVIGATION INTEGRATION FOR KINEMATIC AIRBORNE APPLICATIONS
}

\author{
Ryan Watson
}

\author{
Thesis submitted to the \\ Benjamin M. Statler College of Engineering and Mineral Resources \\ at West Virginia University \\ in partial fulfillment of the requirements for the degree of \\ Master of Science \\ in \\ Aerospace Engineering \\ Jason N. Gross, Chair Ph.D. \\ John A. Christian, Ph.D. \\ Yu Gu, Ph.D. \\ Department of Mechanical and Aerospace Engineering
}

Morgantown, West Virginia

2016

Keywords: Precise Point Positioning, Inertial Navigation, GPS Integration, Real-Time GIPSY-x

Copyright (C) 2016 Ryan Watson 


\section{ABSTRACT \\ Precise Point Positioning Inertial Navigation Integration for Kinematic Airborne Applications}

Ryan Watson

UAVs have the potential for autonomous airborne remote sensing applications that require rapid response to natural hazards (e.g. volcano eruptions, earthquakes). As these applications require very accurate positioning, tightly coupled Global Positioning System (GPS) Precise Point Positioning (PPP) Inertial Navigation Systems (INS) are an attractive method to perform real-time aircraft positioning. In particular, PPP can achieve a level of positioning accuracy that is similar to RealTime Kinematic (RTK) GPS, without the need of a relatively close GPS reference station. However, the PPP method is known to converge to accurate positioning estimate more slowly when compared to RTK, a drawback of PPP that is amplified whenever the receiver platform is faced with GPS challenged environments, such as poor satellite visibility and frequent phase breaks.

This thesis presents the use of a simulation environment that characterizes the position estimation performance sensitivity of PPP/INS through a Monte Carlo analysis that is considered under various conditions: such as, the intensity of multipath errors, the number of phase breaks, the satellite geometry, the atmospheric conditions, the noise characteristics of the inertial sensor, and the accuracy of GPS orbit products. After the PPP/INS formulation was verified in a simulation environment, the INS formulation was incorporated into NASA JPL's Real-Time GIPSY-x. This software was then verified using eight recorded flight data sets provided by the National Geodetic Survey (NGS), National Oceanic and Atmospheric Administration (NOAA) program called Gravity for the Redefinition of the American Vertical Datum (GRAV-D). 


\section{Acknowledgments}

First, I would like to thank my advisor, Dr. Jason Gross, whose support and guidance from the outset of this project was invaluable. Without his input and motivation this project would not have been completed. I would also like to thank the other members of my committee, Dr. John Christian and Dr. Yu Gu for the assistance and feedback that they provided on this project.

Additionally, I would like to thank Dr. Yoaz Bar-Sever at JPL for funding this research and providing useful feedback on GIPSY-OASIS and RTGx. To Dr. Bruce Haines I would like to extend my gratitude for the discussion about kinematic data processing with GIPSY-OASIS. And at NGS, NOAA I would like to thank Carly Weil for providing the flight collected data.

Finally, I would like to thank the West Virginia Space Grant Consortium for granting me a Graduate Fellowship. 


\section{Contents}

$\begin{array}{ll}\text { Abstract } & \text { ii }\end{array}$

Acknowledgments $\quad$ iv

List of Figures $\quad$ viii

List of Tables $\quad x$

1 Introduction $\quad 1$

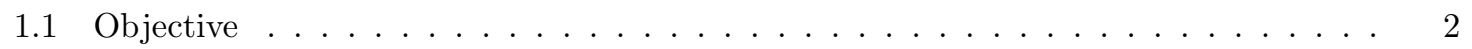

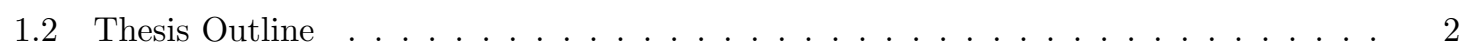

2 GPS 4

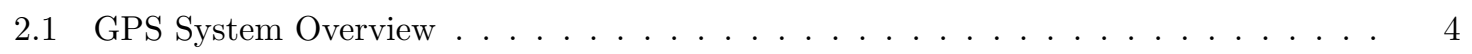

2.1 .1 Space Segment . . . . . . . . . . . . . . . . 4

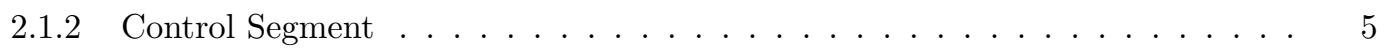

$2.1 .3 \quad$ User Segment . . . . . . . . . . . . . . . . . . . . . . . 5

2.1 .4 Ground Segment . . . . . . . . . . . . . . . . . . . 6

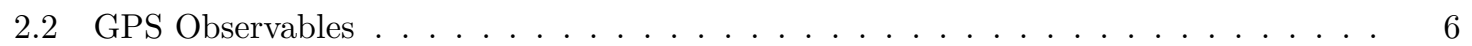

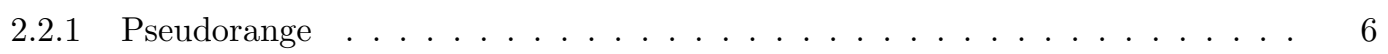

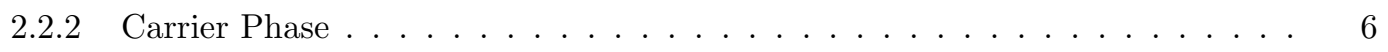

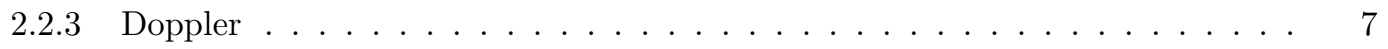

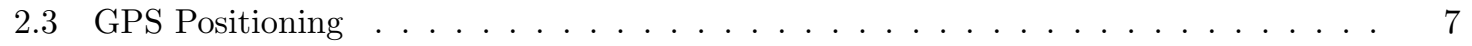

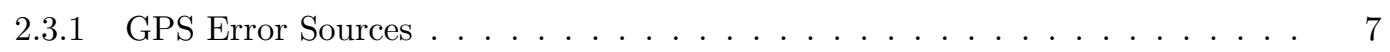

$2.3 .2 \quad$ PPP Observation Model . . . . . . . . . . . . . . . . . . . . . 11 
3 Inertial Navigation 12

3.1 Inertial Measurement Devices Overview . . . . . . . . . . . . . . . . . . 12

3.2 Inertial Measurement Device Common Error Sources . . . . . . . . . . . . . . . . . 13

3.2 .1 Scale Factor . . . . . . . . . . . . . . . . . . . . . 13

3.2.2 Non-orthogonality ........................ 13

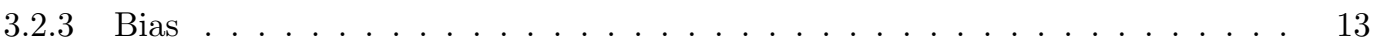

3.2.4 Random Walk ......................... 14

3.3 INS Mechanization . . . . . . . . . . . . . . . . . . . . . . . . 14

3.4 INS Error Model . . . . . . . . . . . . . . . . . . . . . . . . . 17

4 GPS INS Integration $\quad 19$

4.1 Integration Methods . . . . . . . . . . . . . . . . . . . . . . . 20

4.1 Loosely Coupled ......................... 20

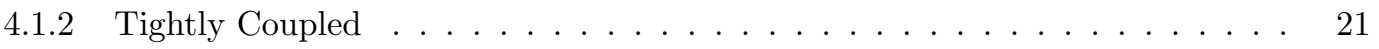

4.1 .3 Deeply Coupled . . . . . . . . . . . . . . . . . . . . 21

4.1.4 Comparison of Integration Methods . . . . . . . . . . . . . . . . . . 21

4.2 Extended Kalman Filter . . . . . . . . . . . . . . . . . . . . . . . . . . . . 22

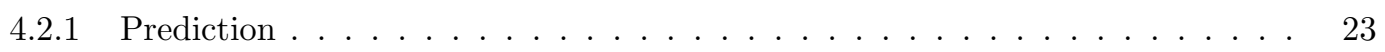

4.2 .2 Measurement Update ... . . . . . . . . . . . . . . 24

4.2.3 Assigning Process and Measurement Noise . . . . . . . . . . . . . . . . . . . 24

4.3 Tightly Coupled Error State Extended Kalman Filter ． . . . . . . . . . . . . . . . . 26

4.4 PPP Measurement Sensitivity Matrix . . . . . . . . . . . . . . . . . . 27

4.5 IMU to GPS Lever Arm . . . . . . . . . . . . . . . . . . . . . . . . . . . 28

4.6 GNSS/IMU Measurement Time-Alignment . . . . . . . . . . . . . . . . . . . 29

5 Simulation Results $\quad 30$

5.1 Simulation Overview ............................ . . 30

5.1 Simulation Inputs . . . . . . . . . . . . . . . . . . 30

5.1 .2 GPS Data Generation ..................... 31

5.1 .3 IMU Data Generation . . . . . . . . . . . . . . . . . . . 32

5.1 .4 Simulation Simplifications . . . . . . . . . . . . . . . . . . 32

5.2 Monte Carlo Design . . . . . . . . . . . . . . . . . . . . . . . . 34

5.3 Results..................................... . . 34

5.3.1 Absolute Positioning Performance . . . . . . . . . . . . . . . . 34 
5.3.2 Sensitivity to PPP Product Quality . . . . . . . . . . . . 39

5.3 .3 Attitude Estimation Performance . . . . . . . . . . . . . . . . . . . 40

5.3 .4 Solution Convergence $\ldots \ldots \ldots \ldots$. . . . . . . . . . . . . . 42

5.3.5 Smoothness of Positioning Solution . . . . . . . . . . . . . . . . . . . . . . 42

6 Flight Data Results $\quad 45$

6.1 GIPSY-OASIS Overview . . . . . . . . . . . . . . . . . . . 45

6.2 RTGx Overview ............................ . . . 45

6.3 Data Sets ............................... . . . 46

6.4 Processing Strageties . . . . . . . . . . . . . . . . . . . . . . . . 48

6.4.1 GIPSY-OASIS Reference Solution Processing Stragety . . . . . . . . . . . . . 48

6.4.2 RTGx Base Processing Stragety . . . . . . . . . . . . . . . . 50

6.5 Results................................ . . . . . . . . . . . . .

6.5.1 Base Run ....................... 51

6.5.2 Linearize About Previous State Estimate . . . . . . . . . . . . . . . . 52

6.5.3 Troposphere Model ....................... 55

6.5.4 Positioning Smoothness ..................... 56

6.5.5 Attitude Estimation . . . . . . . . . . . . . . . . . . . . 57

7 Conclusion $r 61$ 


\section{List of Figures}

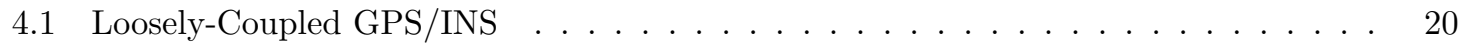

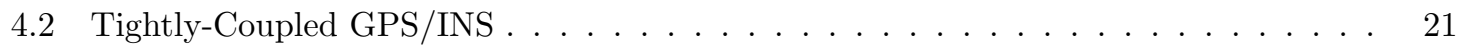

4.3 Kalman Filtering Algorithm Schematic . . . . . . . . . . . . . . . . . . . . . 23

5.1 Flowchart Depicting the Simulation Environment . . . . . . . . . . . . . . . 31

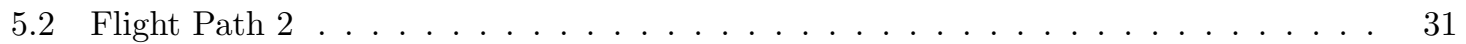

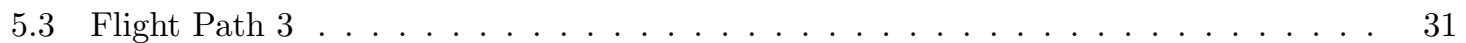

5.4 Attitude Dependent Satellite Masking . . . . . . . . . . . . . . . . . . 32

5.5 Number of Satellites in View for Flight . . . . . . . . . . . . . . . . 32

5.6 Phase Breaks for an Example Flight $\ldots \ldots \ldots \ldots$

5.7 Roll for an Example Flight $\ldots \ldots \ldots$

5.8 Error Between Broadcast Products of IGS and CODE Products . . . . . . . . . . . . 33

5.9 Allan Deviation for Accelerometer and Gyroscope . . . . . . . . . . . . . . . . . . . 33

5.10 Median of Per Flight RMS Error . . . . . . . . . . . . . . . . 35

5.11 Median of Per Flight RMS Error Reduction from INS $\ldots \ldots \ldots$

5.12 CDF of RMS Positioning Error Reduction form INS . . . . . . . . . . . . . . 37

5.13 Median of Per Flight RMS Error Reduction from INS by Flight Path . . . . . . . . . 38

5.14 Median of Per Flight RMS Error Reduction from INS by Number of Phase Breaks . 38

5.15 Median of Per Flight RMS Error Reduction from INS by IMU . . . . . . . . . . . 39

5.16 Median of Per Flight RMS Error Reduction from INS by Magnitude of Troposphere 40

5.17 Median of Per Flight RMS Error Reduction from INS by Magnitude of Multipath . . 40

5.18 Median of Per Flight RMS Error Reduction from INS by PPP Product . . . . . . . . 41

5.19 Example Attitude Estimation Error over a Single Flight . . . . . . . . . . . . . . 41

5.20 Cumulative Attitude Estimation Performance over 100 Flights ～. . . . . . . . . . 42

5.21 Average Absolute Phase Bias Estimation Error . . . . . . . . . . . . . . . . . 43 
5.22 Periodogram of Vertical Position Error for Single Flight . . . . . . . . . . . . . .

6.1 Altitude profile of the d297,y2008 GRAV-D data-set. . . . . . . . . . . . . . . . 47

6.2 Lat/Lon profile of d297,y2008 GRAV-D data-set. . . . . . . . . . . . . . . . . . . . 48

6.3 Kinematic PPP Strategy with GIPSY OASIS. A wrapper software is used to interactively process the position solution. . . . . . . . . . . . . . . . . . . . 49

6.4 CDF of RSS Positioning Error for Base Configuration . . . . . . . . . . . . . . . . 52

6.5 CDF of RSS positioning error without input position time-series using PC-LC ob-

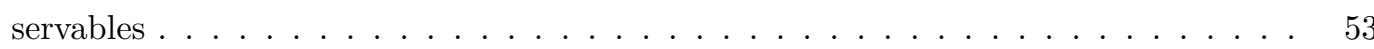

6.6 CDF of RSS positioning error without input position time-series using only PC ob-

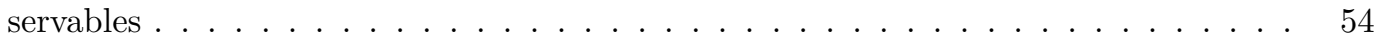

6.7 Max positioning as a function of troposphere model . . . . . . . . . . . . . . 55

6.8 Median of positioning error as a function of troposphere model . . . . . . . . . . 56

6.9 Periodogram of Vertical Position Error for Single Flight . . . . . . . . . . . . . . . . 57

6.10 CDF of total attitude statistics. Includes data from the full duration of all eight data

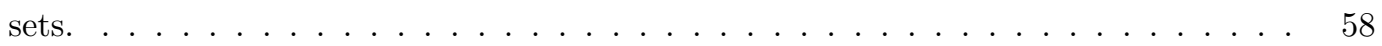

6.11 Navigation Grade IMU Attitude Statistics for Base Run Configuration . . . . . . . . 59

6.12 Tatical Grade IMU Attitude Statistics for Base Run Configuration . . . . . . . . . . 60 


\section{List of Tables}

5.1 Simulation Parameters . . . . . . . . . . . . . . . . . . . . . 34

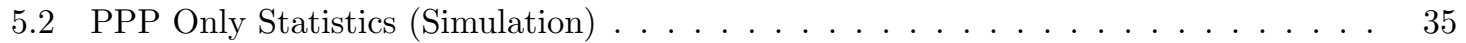

$5.3 \mathrm{PPP} / \mathrm{INS}$ Statistics $($ Simulation $) \ldots \ldots \ldots \ldots \ldots \ldots$

$5.4(\mathrm{PPP}-\mathrm{PPP} / \mathrm{INS})$ Statistics (Simulation) $\ldots \ldots \ldots \ldots \ldots$

6.1 Flight Collected Data Set Durations _ . . . . . . . . . . . . . . . . . . . 46

6.2 GPS/INS System for Flight Collected Data . . . . . . . . . . . . . . . . . 47

6.3 Statistics of positioning performance for full baserun configuration (flight data) . . . 52

6.4 Statistics of positioning performance after convergence for baserun configuration (flight

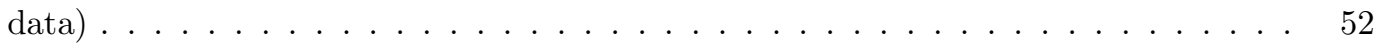

6.5 Statistics of positioning performance for full PC-LC previous epoch linearization configuration (flight data) $\ldots \ldots \ldots \ldots \ldots \ldots \ldots \ldots$

6.6 Statistics of positioning performance after convergence for previous epoch linearization configuration $($ flight data $) \ldots \ldots \ldots \ldots \ldots \ldots$

6.7 Statistics of positioning performance for full PC-only previous epoch linearization configuration (flight data) $\ldots \ldots \ldots \ldots \ldots \ldots \ldots$

6.8 Statistics of positioning performance for last-half of previous epoch linearization con-

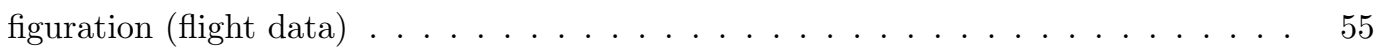

6.9 Statistics of positioning performance for different troposphere models (flight data) . 56

6.10 Statistics of attitude performance for all flight data with baserun configuration (flight

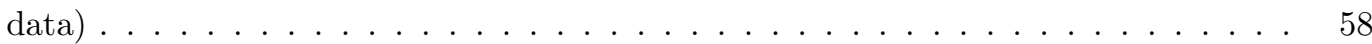

6.11 Statistics of attitude performance for navigation grade IMU for all fligth data with baserun configuration (flight data) _ . . . . . . . . . . . . . . . . 59

6.12 Statistics of attitude performance for tatical grade IMU for all flight data with base run configuration (flight data) $\ldots \ldots \ldots \ldots \ldots \ldots \ldots$ 


\section{Acronyms}

\begin{tabular}{|c|c|}
\hline ARW & Angular Random Walk \\
\hline C/A-code & Coarse-Acquisition Code \\
\hline DCM & Direction Cosine Matrix \\
\hline DGPS & Differential GPS \\
\hline $\mathrm{ECEF}$ & Earth Centered Earth Fixed \\
\hline ECI & Earth Centered Inertial \\
\hline EKF & Extended Kalman Filter \\
\hline GIPSY-OASIS & GNSS-Inferred Positioning System and Orbit Analysis Simulation Software \\
\hline GMF & Global Mapping Function \\
\hline GPS & Global Positioning System \\
\hline GPT2 & Global Pressure and Temperature model \\
\hline GRAV-D & Gravity for the Redefinition of the American Vertical Datum \\
\hline IF & Ionospheric-free \\
\hline IMU & Inertial Measurement Unit \\
\hline INS & Inertial Navigation System \\
\hline $\mathrm{KF}$ & Kalman Filter \\
\hline$L_{1}$ & 1575.42 MHz GPS frequency \\
\hline$L_{2}$ & 1227.60 MHz GPS frequency \\
\hline MEMS & Microelectromechanical Systems \\
\hline NASA & National Acronym Slinging Society \\
\hline NGS & National Geodetic Survey \\
\hline NOAA & National Oceanic and Atmospheric Administration \\
\hline P-code & Precise Code \\
\hline PPP & Precise Point Positioning \\
\hline PRN & Pseudo-Random Noise \\
\hline RMS & Root Mean Square \\
\hline RSS & Root of Sum of Squares \\
\hline RTGx & Real-Time Gipsy-x \\
\hline RTK & Real-Time Kinematic \\
\hline STM & System Transformation Matrix \\
\hline VRW & Velocity Random Walk \\
\hline VMF1 & Vienna Mapping Function \\
\hline WVU & West Virginia University \\
\hline
\end{tabular}




\section{Nomenclature}

\begin{tabular}{|c|c|c|}
\hline Symbol & Units & Description \\
\hline $\mathrm{x}$ & & State Vector \\
\hline$\psi$ & radians & Attitude \\
\hline$v$ & meters/second & Velocity \\
\hline$r$ & meters & Position \\
\hline$R$ & meters & Geometric Range \\
\hline$C$ & & Coordinate Transformation Matrix \\
\hline$\rho$ & meters & Pseudorange \\
\hline$\phi$ & meters & Carrier-Phase \\
\hline$c$ & meters/second & Speed of light \\
\hline$\delta \theta$ & radians & Delta Angle \\
\hline$\delta v$ & meters/second & Delta Velocity \\
\hline$e l$ & radians & User to Satellite Elevation Angle \\
\hline$F$ & & System Matrix \\
\hline$\Phi$ & & State Transition Matrix \\
\hline$\tau$ & seconds & Time Step \\
\hline$\phi$ & radians & Roll \\
\hline$\theta$ & radians & Pitch \\
\hline$\psi$ & radians & Yaw \\
\hline$\omega_{e}$ & radians/second & Earth Rotation Rate \\
\hline Lat & radians & Latitude \\
\hline Lon & radians & Longitude \\
\hline$H$ & meters & Height \\
\hline
\end{tabular}




\section{Chapter 1}

\section{Introduction}

The Precise Point Positioning (PPP) approach uses dual-frequency undifferenced GPS observables in conjunction with precise orbit and clock bias products and measurement models to achieve decimeter to centimeter level positioning with a single receiver [1]. Real-time PPP is enabled by orbit and clock products being broadcast to end user. These global correctors have been integrated into the L-band by commercial entities[2], can be obtained over the internet or through an Iridium modem link from NASA's Global Differential GPS System[3], and have recently been made freely available on the internet by the International GNSS Service [4]. The similar level of accuracy of real-time PPP compared to traditional Differential GPS (DGPS) (i.e. Real Time Kinematic) without the need for a nearby static GPS reference station, is particularly attractive for airborne scientific instrument platforms that require this level of accuracy, global coverage and rapid response capability. For instance, real-time PPP is used for NASA's airborne Synthetic Aperture Radar (SAR) platforms that are used for repeat pass interferometry in response to natural hazards such as volcano eruptions, tsunamis, and large earthquakes [5]. The benefits of coupling GPS with Inertial Navigation Systems (INS) have long been well understood[6], and recent studies have investigated and demonstrated combined PPP/INS architectures for kinematic applications on ground vehicles[7], [8], [9], [10], and airborne platforms[10]. In these previous works, both loosely-coupled and tightly-coupled architectures have been investigated, and when provided clear open sky-access and on vehicles with docile dynamics, both approaches have shown to exhibit similar estimation performance[9], [10]. However, when confronted with challenging GPS environments, such as poor visibility (e.g. urban canyon), frequent

phase breaks, and high-multipath, tightly-coupled formulations inherently have the advantage [9], [10]. 


\subsection{Objective}

This thesis has 2 objectives. The first objective is to develop and characterize the sensitivity of PPP/INS for kinematic airborne applications within a simulation environment. For this to be realized, first, a simulation environment is developed within MATLAB. Next, a tightly-coupled PPP/INS error-state Kalman filter is developed. This tighly-coupled PPP/INS error-state Kalman filter was considered under various conditions: the number of phase breaks, the satellite geometry, the atmospheric conditions, the noise characteristics of the inertial sensor, and the accuracy of GPS orbit products.

The validated INS formulation was then incorporated into NASA JPL's Real-Time GIPSY-x (RTGx) software [11]. This leads to the second objective, the validation of the formulation using recorded flight data sets provided by the National Geodetic Survey (NGS), National Oceanic and Atmospheric Administration (NOAA) program called Gravity for the Redefinition of the American Vertical Datum (GRAV-D) [12] [13]. A reference solution for the eight data sets is generated using an iterative processing strategy with GIPSY-OASIS II [14]. Using these reference solutions, a sensitivity analysis of RTGx is conducted. The purpose of this sensitivity study is to see when INS truly with positioning estimation for kinematic PPP/INS applications.

\subsection{Thesis Outline}

The second chapter of this thesis provides an overview of the GPS navigation system and the PPP approach. First, a broad overview of the GPS system is provided. Then the GPS observables are discussed. Finally, a brief discussion of GPS positioning is provided, which includes the PPP observation model. Chapter Three of this thesis covers Inertial navigation. This chapter starts with an overview of inertial measurement devices. Then a detailed discussion of INS mechanization in the Earth Cenerted Inertia (ECI) frame is provided. Lastly, the INS error model formulation is discussed. The fourth chapter covers GPS/INS integration methods. This section provides an overview of the two integration methods commonly used for aerospace applications. Then, a brief overview of the Extend Kalman Filter algorithm is provided. And finally, the integration method utilized in this research. With the preliminary information provided in the previous chapters, chapter five covers the use of a simulation environment to characterize the K-PPP/INS integration algorithm. First, a discussion of the simulation environment is discussed. Then the simulation results obtained are provided. Chapter six provides the processing of eight real data sets using RTGx. Within this 
chapter is an overview of the RTGx software. Then, using eight flight collected data sets, the sensitivity of RTGx is evaluated under several conditions. Finally, chapter seven provides some concluding remarks and plans for future work. 


\section{Chapter 2}

\section{GPS}

Although GPS can be used for several other application ( e.g. the detection of underground nuclear explosions [15], the early detection of tsunamis [16], precise timing [17], and the detection of snow depth [18] ), this thesis is concerned with the use of GPS as a positioning sensor. The ability to use GPS for positioning is based up trilateration: a method of determining position that utilizes time of flight measurements ( i.e. pseudorange observables ) from at least four satellites [19]. This is because the first 3 satellites are used to narrow the location of the receiver down to two locations. The forth satellite is used to select the right location and calculate timing and location corrections. For accurate positioning the receiver must compensate for common GPS error sources. This chapter will cover a brief overview of the GPS System which will include a discussion of the GPS observables, the common GPS error sources, and the observation model that is used to calculate the GPS observables.

\subsection{GPS System Overview}

The GPS system can be broken into four distinct sections: the space segment, the control segment, the user segment, and the ground segment. This section will detail each of those segments in detail.

\subsubsection{Space Segment}

\section{Orbit Design}

As stated previously, GPS point positioning requires there to be at least four satellites in view. With this is mind, the GPS orbit was designed. There are nominally twenty four GPS satellites in orbit. These twenty four satellites are distributed evenly over six orbital planes where each plane is at an 
inclination angle of $55^{\circ}$ at an almost circular orbit with a radius of 20,200 kilometers [19]. As a consequence of the GPS orbit design, each GPS satellite makes two full revolutions around the earth in 23 hours and 58 minutes (i.e. one sidereal day ). This means that each satellite appears over the same location every every sidereal day, which is more commonly called "repeating ground-tracks" [20].

\section{Signal Structure}

Every GPS satellite transmits on at least two L-band radio frequencies: the Link $1\left(L_{1}\right)$ frequency and the Link $2\left(L_{2}\right)$ frequency, which are centered about $1.575 \mathrm{GHz}$ ( corresponds to a wave length of $19 \mathrm{~cm}$ ), and $1.227 \mathrm{GHz}$ ( corresponds to a wave length of $24.4 \mathrm{~cm}$ ), respectively [19]. Modulated on the $L_{1}$ frequency is the Coarse-Acquisition Code (C/A-code), which has a frequency of 1.023 MHz. In addition to the $\mathrm{C} / \mathrm{A}$-code, a navigation message is also modulated onto the $L_{1}$ carrier. This navigation messages provides the user with information about the satellites health status, ephemeris, and clock bias. Modulated on $L_{1}$ and $L_{2}$ is the Precise Code (P-code), which has a frequency of 10.23 M Hz. From the information provided ( the carrier, the PRN, and the navigation message ), the user can extract three observables: the pseudorange observable, the carrier-phase observable, and the doppler observable [20].

\subsubsection{Control Segment}

The GPS control segment is composed of a network of seventeen monitoring stations operated by the Air Forec and the National Geospatial-Intelligence Agency (NGA). These stations constantly monitor the satellites and report back to the Master Control Station, located at Schriever Air force Base in Colorado Springs. The data processed at the Master Control Station is used to generate the orbit and clock errors and is uplinked to the GPS satellites on a $1.783 \mathrm{GHz}$ signal [20].

\subsubsection{User Segment}

The user segment consists of various GPS receiver equipment, which receive the signals from the GPS satellites and use the received information to calculate the users position and the GPS system time. 


\subsubsection{Ground Segment}

The ground segment is composed of civilian tracking networks that provide the user segment with precise ephemeris information. This precise ephemerids information is what enables PPP. Additionally, these global correctors have been integrated into the L-band by commercial entities [2], can be obtained over the internet or through an Iridium modem link from NASA's Global Differential GPS System [3], and have recently been made freely available on the internet by the International GNSS Service [4].

\subsection{GPS Observables}

There are three GPS observables: the pseudorange, the carrier-phase, and the doppler observable. These three measurements will be described in greater detail in this section.

\subsubsection{Pseudorange}

The first observable that will be discussed is the pseudorange. This observable is essentially a measure of the propagation time of the signal scaled by the speed that the wave is propagated. Where the propagation time is obtained through correlating the PRN that is propagated by the satellite to the PRN generated on the receiver. In addition, there will be a clock bias because it is cost prohibitive for users to have a more precise clock. This clock bias is where the term pseudorange comes from. The term pseudorange signifies that the observable is the true range distorted by the clock bias and additional error sources.

The equation to calculate the pseudorange can be found in Eq. 2.1: where $t_{r}$ is the time the signal was received, $t_{s}$ is the time that the satellite propogated the signal, $c$ is the speed of light, $b$ is a composite clock bias composed of both satellite and a receiver clock bias, and $\epsilon$ contains all additional error sources. These additional error sources are described in the next section.

$$
\rho^{j}=\left(t_{r}-t_{s}^{j}\right) c+b^{j}+\epsilon^{j}
$$

\subsubsection{Carrier Phase}

The carrier-phase observable can be though of as a very similar measurement to the pseudorange observable. However, there are two large caveats: the first being, that the carrier-phase observable is orders of magnitude more precise than the pseudorange observable, and the second being that 
the carrier-phase observable contains an ambiguous number of wave lengths that must be resolved before the precision of the observable can be utilized.

A simplified carrier-phase observation model can be seen in Eq. 2.2: where, $\lambda$ is the wave length associated with the propagation frequency, $R$ is the geometric distance between the satellite and the receiver, $N$ is the ambiguity term associated with the carrier-phase data, $b$ is a composite clock bias composed of both a receiver and a satellite component, and $\epsilon$ contains all additional un-modelled error sources.

$$
\Phi^{j}=R^{j}+\lambda N^{j}+b^{j}+\epsilon^{j}
$$

\subsubsection{Doppler}

The doppler shift or relative motion of a satellite with respect to a receiver results in a change in the observed frequency. If the satellites velocity is known, then this observable can be used to estimate the receivers velocity [19].

The doppler shift can be calculated using Eq. 2.3. This equation is the same as Eq. 2.1 with the exception that the time derivative of each component is taken.

$$
\dot{\rho}^{j}=\dot{R}^{j}+\dot{b}^{j}+\dot{\epsilon}^{j}
$$

\subsection{GPS Positioning}

\subsubsection{GPS Error Sources}

The common GPS error sources can be broken in three catagories. The first error source is contributed to the control segment. These errors are either associated with the estimated location of the satellite or the satellite clock bias solution. The next group of GPS errors are associated with the propagation medium. This group of errors are either due to the ionosphere or the troposphere. The final group of errors are contributed to the measurement errors and are generally the hardest to mitigate.

\section{Space/Control Segment}

The control segment is composed of several monitoring stations that collect data from the satellites and send the satellites updated clock and ephemeris information. This updated navigation infor- 
mation is estimated using a Kalman filter at the master control station. This corrected ephemeris information is broadcast to the user and is what enables PPP.

Ephemeris One error that is associated with the control segment is the ephemeris error. The ephemeris error is the amount of error associated with the satellites estimated position. This error is associated with several factors, such as, solar radiation pressure, yaw-bias, and aerodynamic drag [21]. This error is generally decomposed into a radial, in-track and cross-track component. Of these tree components, the radial component is generally the smallest but has the largest impact on the user's positioning estimate [22].

Clock Another error associated with the control segment is the satellite clock error. Even though the clocks on the GPS satellites are highly stable there can still be a large error associated with the accumulated satellite clock error. Because of this, the control segment monitors the satellite clocks with respect to GPS standard time. This error is generally decomposed into three components: the satellite clock bias, the satellite clock drift, and the satellite clock drift. To correct this error the control segment broadcasts clock corrects with the navigation message.

Phase Wind-up Due to the nature of the signal being propagated from the GPS satellite (i.e. right circularyly polarized [19]), the carrier-phase observable is dependant upon the orientation of the receiver and satellite antennas. This affect can account for up to one wavelength of error, and is commonly known as phase wind-up [23].

\section{Propogation Medium}

Due to the altitude or the GPS orbit, roughly $20,200 \mathrm{~km}$, there are two propagation medium errors: the ionosphere and the troposphere.

Ionosphere The error associated with the ionosphere is attributed to the total electron content along the path of the propagated signal [19]. Fortunately, the ionosphere is a dispersive medium, which means that the amount that the ionosphere affects a signal propagated though it is dependent upon the frequency of the signal. So to mitigate the ionospheric delay, the dispersive nature of the medium is leveraged, and a linear combination of the GPS $L_{1}$ and $L_{2}$ frequencies $(1575.42 \mathrm{MHz}$ and $1227.60 \mathrm{MHz}$, respectively) is formed to produce ionospheric-free (IF) pseudorange and carrier phase measurements[19]. The IF combination for psuedorange and carrier-phase can be seen in Eq. 
2.4 and Eq. 2.5, respectively.

$$
\begin{gathered}
\rho_{I F}^{j}=\rho_{L 1}^{j}\left[\frac{f_{1}^{2}}{f_{1}^{2}-f_{2}^{2}}\right]-\rho_{L 2}^{j}\left[\frac{f_{2}^{2}}{f_{1}^{2}-f_{2}^{2}}\right]=2.546 \rho_{L 1}^{j}-1.546 \rho_{L 2}^{j} \\
\phi_{I F}^{j}=\phi_{L 1}^{j}\left[\frac{f_{1}^{2}}{f_{1}^{2}-f_{2}^{2}}\right]-\phi_{L 2}^{j}\left[\frac{f_{2}^{2}}{f_{1}^{2}-f_{2}^{2}}\right]=2.546 \phi_{L 1}^{j}-1.546 \phi_{L 2}^{j}
\end{gathered}
$$

In Eq. 2.4 and Eq. 2.5 the $f_{1}$ and $f_{2}$ are the $L_{1}$ and $L_{2}$ frequencies, $\rho_{L 1}$ and $\rho_{L 2}$ are the pseudorange measurements on the $L_{1}$ and $L_{2}$ frequencies, $\phi_{\boldsymbol{L} \mathbf{1}}$ and $\phi_{\boldsymbol{L} \mathbf{2}}$ are the carrier-phase measurements on the $L_{1}$ and $L_{2}$ frequencies. The superscript $j$ in Eq. 2.4 and Eq. 2.5 is used to designate the measurement between the platform and satellite $j$.

Troposphere Unfortunately, troposphere is not a dispersive medium for the $L_{1}$ or $L_{2}$ carriers, so it cannot be eliminated by simply constructing a combination of the $L_{1}, L_{2}$ observables. Additionally the troposphere is highly temporal as it is a function of temperature, pressure, and humidity. So, the troposphere is generally modeled as two separate components: the dry component and the wet component. These components are estimated in the zenith direction using a troposphere model and mapped to the specific satellite using the elevation angle between the receiver and the satellite. This concept is show in Eq. 2.6: where $m_{d}$ and $m_{w}$ are elevation angle dependent troposphere error scalings for the dry and wet components, respectively; $T_{z, d}$ is the zenith troposphere delay associated with the dry component; $T_{z, w}$ is the zenith troposphere delay associated with the wet component.

$$
T^{j}=m_{d}\left(e l^{j}\right) T_{z, d}+m_{w}\left(e l^{j}\right) T_{z, w}
$$

There are several models used to estimate the troposphere delay. These models can be decomposed into two classes: empirical (i.e. GMF), or based upon data generated with numerical weather models (i.e. VMF1). The next few paragraphs will be used to describe the troposphere models used in this thesis.

As an example, two simple troposphere models are shown. The first model is a solely dependent upon the height of the platform, and is only used to estimate the hydrostatic (i.e. dry) component of the delay associated with the troposphere. This model can be seen in Eq. 2.7: where $h$ is the height above the ellipsoid in meters.

$$
T_{z, d}=1.013 * 2.27 * e^{-0.000116 h}
$$


The next example nominal troposphere estimation model was developed by Saastamoinen [24]. This model is used to generate both the wet and dry delay. The dry delay can be calculated using Eq. 2.8: where $\phi$ is the platforms latitude, $h$ is the platforms height above the ellipsoid in meters, and $P_{o}$ is the total pressure in millibars. The wet delay can be calculated using Eq. 2.9. In Eq. 2.9, $T_{o}$ is the temperature in kelvin, and $e_{o}$ is the partial pressure due to the water vapor in millibars.

$$
\begin{gathered}
T_{z, d}=0.002277(1+0.0026 \cos 2 \phi+0.00028 h) P_{o} \\
T_{z, w}=0.002277\left(\frac{1255}{T_{o}}+0.05\right) e_{o}
\end{gathered}
$$

In addition to these two models, three additional models were used in this research. The first model is the Hopfield model [25]. This is the model that was used within the simulation. The next two models are used within Real-Time GIPSY-x (RTGx), and unlike the previous models, are derived from numerical weather models. The first model is the Global Pressure and Temperature Model (GPT2) [26]. The final troposphere model used is this research is the Vienna Mapping Function (VMF1) [27].

With the zenith troposphere delay estimated, there is the need to scale the delay as a function of the satellites position with respect to the receiver. There are several mapping functions which perform this task. The three that are used within this thesis are : the Niell mapping function [28], the GMF [29], and the VMF1 [27]. Where it should be noted that each of these mapping functions assume azimuthal symmetry (i.e. they are solely dependent upon the elevation angle of the satellite).

\section{Measurement Errors}

This group of GPS errors are receiver/antenna design, and code structure. This is unlike all of the previous error sources, which were the same for both the pseudorange and carrier-phase.

Multipath Multipath is an error induced upon the receiver by reflected GPS signals reaching the receivers antenna [30]. The reflected signal is always delayed and generally significantly weaker than the direct line-of-sight signal. These reflected signals sum with the original signal to form a noisy observable. This noise affects the pseudorange and the carrier-phase; however, the affect is significantly smaller for the carrier-phase observable than the pseudorange observable [31]. 
Receiver Thermal noise The most fundamental kind of noise created within electrical circuitry is caused by the random motion of electrons [32]. This type of noise is generally called thermal noise. And because there is no correlation two receivers, this is one of the few GPS error sources that is not mitigated through data differencing [19].

Phase Center Variations The location where the GPS signal is received, known as the phase center, in general is not the geometric center of the antenna. Not only is this value not the geometric center of the antenna but also varies dependent upon the orientation of the satellite with respect to the receiver and the frequency of the signal propagated [33].

\subsubsection{PPP Observation Model}

The PPP approach utilizes dual-frequency undifferenced GPS observables. Because undifferenced data is being used, methods for mitigating GPS error sources (e.g., ionospheric delay, tropospheric delay, and receiver clock delay), which are canceled through data differencing with traditional Differential GPS (DGPS), must be incorporated in the measurement models. Using the IF combination, the pseudorange and carrier-phase measurements are modeled as shown in Eq. 2.10 and Eq. 2.11, respectively: where $\delta t_{u}$ is the receiver's clock bias, $T_{z}$ is the tropospheric delay in the zenith direction, $m\left(e l^{j}\right)$ is a user to satellite elevation angle dependent mapping function, $\lambda_{I F}$ is the wavelength corresponding to the IF combination, and $N_{I F}$ is phase ambiguity. The geometric range between the platform and the satellite is denoted as $R^{j}$, and given as $R^{j}=\sqrt{\left(x^{j}-x_{u}\right)^{2}+\left(y^{j}-y_{u}\right)^{2}+\left(z^{j}-z_{u}\right)^{2}}$, where the subscript $u$ represents the platforms position, and the superscript $j$ represents the satellite, both in the same Cartesian reference frame.

$$
\begin{gathered}
\rho_{I F}^{j}=R^{j}+c \delta t_{u}+T_{z} m\left(e l .^{j}\right)+\epsilon_{\rho}^{j} \\
\phi_{I F}^{j}=R^{j}+c \delta t_{u}+T_{z} m\left(e l^{j}\right)+\lambda_{I F} N_{I F}^{j}+\epsilon_{\phi}^{j}
\end{gathered}
$$

In Eqs. 2.10 and 2.11 the remaining un-modeled error sources are indicated with $\epsilon$. In addition, the tropospheric delay $T_{z}$ in Eqs. 2.10 and 2.11 is composed of both a wet and dry components. In practice, the dry delay makes up the majority of the total zenith path tropospheric delay (i.e. $\approx$ 2.5 meters) and can be well modeled. The wet delay is on the order to $10 \%$ the dry delay and is typically estimated. 


\section{Chapter 3}

\section{Inertial Navigation}

Parts of this chapter are reproduced from conference papers "Performance Characterization of Tightly-Coupled GNSS Precise Point Positioning Inertial Navigation within a Simulation Environment" [34], "Integration of Inertial Navigation into Real-Time GIPSY-x (RTGx)" [35]

Sometimes the acronyms IMU and INS are used interchangeably; however, in this thesis, an IMU is used to denote a set of of three orthogonal accelerometers and three orthogonal gyroscopes while INS is the processing of IMU data on a navigation computer. The rest of this chapter is organized as follows: first, a brief overview of inertial measurement devices will be provided; then, common errors to inertial devices will be discussed; next, the mechanization of the IMU data is covered; finally, the error state INS formulation will be provided in the inertial frame.

\subsection{Inertial Measurement Devices Overview}

As stated above, the IMU is composed of three accelerometers and three gyroscopes organized in a triad. For this thesis, it is assumed that the IMU is rigidly mounted to the body of the platform. This IMU configuration is commonly called a strapdown system. With a strapdown INS system, the accelerometers are used to measure the specific force in the body-frame, which is also the nongravitational acceleration of the platform. This concept is depicted in Eq. 3.1: where $f$ is the measured specific force, $a$ is the body's acceleration, and $g$ is the gravitational acceleration. The IMU cannot measure gravity so the gravitational term can easily be corrected for by using a gravity 
model.

$$
f=a-g
$$

Because the specific force is measured in the body-frame, the accelerometer measurements cannot simply be integrated twice to estimate the platforms position. Instead, the gyroscopes measured angular information must be utilized to rotate the accelerometers measurements into the desired frame where the position and velocity can then be estimated. This processed is generally called the mechanization of an IMU and is described in greater detail later in this chapter.

\subsection{Inertial Measurement Device Common Error Sources}

\subsubsection{Scale Factor}

A scale factor error can be thought of as a linear scaling of the input. In a sensor absent of a scale factor error, the ratio formed by the input and output signal would equal one; however, that is generally not the case. In reality the output of the sensor is generally proportional to the input but scaled by some constant factor.

\subsubsection{Non-orthogonality}

As stated above, an IMU is composed of three accelerometers and three gyroscopes mounted orthogonally. However, due to manufacturing limitations, the sensors are never perfectly mounted orthogonally. This non-orthogonality of the sensor set leads to a correlations in the measurements. Luckily, this error can usually be dramatically reduced through a careful calibration.

\subsubsection{Bias}

The easiest way to conceptualize bias is to consider an IMU firmly placed on a level surface. In this configuration, the accelerometer with its sensitivity axis in the vertical direction will measure the gravitational acceleration. That is, the accelerometer should measure $9.81 \mathrm{~m} / \mathrm{s}$; however, if there is a bias present, the reported output will be the summation of the real output and the bias term, which can be seen in Eq. 3.2.

$$
x=S f(x)+b
$$

In Eq. $3.2, S$ is a scale factor and $b$ is the bias term. 


\subsubsection{Random Walk}

The random walk model is used quite frequently in state estimation. This model assumes that at each time step the variable takes a random step away from its previous value, and the steps are independent [36]. In this section, two random walk processies will be discussed and how they apply to the Kalman filters process noise will be described.

Angular Random Walk It is known that the output of a MEMS gyroscope is altered by thermal noise which fluctuates at a much higher rate that the sampling rate of the sensor [37]. This high rate thermal noise causes the sampled data to be perturbated by white noise. The integration of a white noise process causes a random walk process [36]. The integration of the output of the gyroscope to calculate attitude produces a random walk error, commonly denoted as angular random walk (ARW).

The ARW term is applied to the process noise of the Kalman filter as the amount of uncertienty in the gyroscope bias term.

Velocity Random Walk As stated above, the output from a MEMS accelerometer is altered by a white noise sequence. The output of the accelerometer must be integrated to calculate velocity, so the white noise on the output of an accelerometer creates a velocity random walk (VRW).

The VRW term is applied to the process noise of the Kalman filter as the amount of uncertienty in the accelerometer bias term.

\subsection{INS Mechanization}

The mechanization of the IMU data is composed of four steps: the attitude update, the specific force transformation, the velocity update, and the position update. The section details the mechanization in the Earth-Centered Inertial Frame (ECI).

\section{Attitude Update}

To perform the attitude update, a third order Runge-Kutta method for quaternion integration, provided by Jekeli[38], is used in order to limit the algorithmic integration errors to the fourth order. The quaternion is updated using Eq. 3.3: where $I$ is a $4 \mathrm{x} 4$ identity matrix, $\beta$ is a matrix composed of the delta angles measured with the gyroscopes and can be seen in Eq. 3.4, the subscript 
$t$ signifies the discrete samples (e.g. $t-2$ is using data from two time steps previous) .

$$
\hat{q}_{t}=\left[I+\frac{1}{12}\left(\hat{B}_{t}+4 \hat{B}_{t-1}+\hat{B}_{t-2}\right)+\frac{1}{12}\left(I+\frac{1}{4} \hat{\beta}_{t}\right) \hat{\beta}_{t-1} \hat{\beta}_{t-2}+\frac{1}{12} \hat{\beta}_{t}\left(\hat{\beta}_{t-1}-\frac{1}{2} \hat{\beta}_{t-2}\right)\right] \hat{q}_{t-2}
$$

In Eq. $3.4 n$ takes an integer value of 1, 2, or 3 .

$$
\hat{\beta}_{t+1-n}=\left[\begin{array}{cccc}
0 & \left(3\left(\delta \theta_{1}\right)_{t+1-n}-\left(\delta \theta_{1}\right)_{t-n}\right) & \left(3\left(\delta \theta_{2}\right)_{t+1-n}-\left(\delta \theta_{2}\right)_{t-n}\right) & \left(3\left(\delta \theta_{3}\right)_{t+1-n}-\left(\delta \theta_{3}\right)_{t-n}\right) \\
\left(-3\left(\delta \theta_{1}\right)_{t+1-n}+\left(\delta \theta_{1}\right)_{t-n}\right) & 0 & \left(3\left(\delta \theta_{3}\right)_{t+1-n}-\left(\delta \theta_{3}\right)_{t-n}\right) & \left(-3\left(\delta \theta_{2}\right)_{t+1-n}+\left(\delta \theta_{2}\right)_{t-n}\right) \\
\left(-3\left(\delta \theta_{2}\right)_{t+1-n}+\left(\delta \theta_{2}\right)_{t-n}\right) & \left(-3\left(\delta \theta_{3}\right)_{t+1-n}+\left(\delta \theta_{3}\right)_{t-n}\right) & 0 & \left(3\left(\delta \theta_{1}\right)_{t+1-n}-\left(\delta \theta_{1}\right)_{t-n}\right) \\
\left(-3\left(\delta \theta_{3}\right)_{t+1-n}+\left(\delta \theta_{3}\right)_{t-n}\right) & \left(3\left(\delta \theta_{2}\right)_{t+1-n}-\left(\delta \theta_{2}\right)_{t-n}\right) & \left(-3\left(\delta \theta_{1}\right)_{t+1-n}+\left(\delta \theta_{1}\right)_{t-n}\right) & 0
\end{array}\right]
$$

The platform's body to inertial direction cosine matrix (DCM) is related to the updated quaternion of the form $\hat{q}_{t}=[a, b, c, d]^{T}$ using Eq. 3.5. It should be noted that the quaternion needs to be normalized in order to maintian the orthonormal characteristic of the DCM.

$$
C_{b}^{i}=\left[\begin{array}{ccc}
a^{2}+b^{2}-c^{2}-d^{2} & 2(b c+a d) & 2(b d-a c) \\
2(b c-a d) & 2-b^{2}+c^{2}-d^{2} & 2(c d+a b) \\
2(b d+a c) & 2(c d-a b) & a^{2}-b^{2}-c^{2}+d^{2}
\end{array}\right]
$$

\section{Specific Force Transformation}

After the attitude has been updated, the next step in the INS mechanization is the transformation of the accelerometer measured specific force, or equivalently, incremental changes to the body-axis velocity $\Delta v_{b}$ into the inertial frame. This must be done because the accelerometers measures specific force along the body-axis; however, for the velocity update step the specific force must be in the inertial frame. The transformed $\Delta v_{b}$ can be found using Eq. 3.6 where $C_{b}^{i}$ is the body to inertial DCM of the INS updated quaternion from Eq. 3.5.

$$
\Delta v^{i}=C_{b}^{i} \Delta v^{b}
$$

\section{Velocity Update}

With the transformed specific force, the updated velocity is calculated using Eq. 3.7. Where the updated velocity is the summation of the previous velocity value $(k-1 \mid k-1)$, the IMU measured change in velocity, $\Delta v^{i}$, and the acceleration due to gravity in the inertial frame, $\gamma^{i}$, integrated over the sampling interval $\tau$.

$$
v_{k \mid k-1}^{i}=v_{k-1 \mid k-1}^{i}+\Delta v^{i}+\gamma^{i} \tau
$$




\section{Position Update}

The final step in the INS mechanization is to calculate the updated position. The updated position estimate is a function of the previous position estimate and the average of the previous and updated velocity estimates integrated over the sampling interval.

$$
r_{k \mid k-1}^{i}=r_{k-1 \mid k-1}^{i}+\left(v_{k \mid k-1}^{i}+v_{k-1 \mid k-1}^{i}\right) \frac{\tau}{2}
$$

\section{Attitude Transformation}

In most aerospace applications, it is useful to represent the platform attitude with respect to a locally-level NED navigation frame. However, in the presented INS mechanization, a quaternion is used to represent the platform's attitude with respect to the Earth Centered Inertial (ECI) frame. To calculate the body-to-navigation Euler angles, the DCM must be transformed into the navigation frame. This is done using Eq. 3.9, where the Earth-Centered-Earth-Fixed (ECEF) to locally-level NED navigation,$C_{e}^{n}$, and ECEF to inertial, $C_{e}^{i}$, transformations are defined in Eq. 3.10 and Eq. 3.11 , respectively, which can be found in numerous texts [39, 38].

$$
\begin{gathered}
C_{b}^{n}=C_{e}^{n} C_{e}^{i} C_{b}^{i} \\
C_{e}^{n}=\left[\begin{array}{ccc}
-\sin (\text { Lat }) \cos (\text { Lon }) & -\sin (\text { Lon }) & -\cos (\text { Lat }) \cos (\text { Lon }) \\
-\sin (\text { Lat }) \sin (\text { Lon }) & \cos (\text { Lon }) & -\cos (\text { Lat }) \sin (\text { Lon }) \\
\cos (\text { Lat }) & 0 & -\sin (\text { Lat })
\end{array}\right] \\
C_{e}^{i}=\left[\begin{array}{ccc}
\cos \left(\omega_{i e}\left(t-t_{0}\right)\right. & -\sin \left(\omega_{i e}\left(t-t_{0}\right)\right. & 0 \\
\sin \left(\omega_{i e}\left(t-t_{0}\right)\right. & \cos \left(\omega_{i e}\left(t-t_{0}\right)\right. & 0 \\
0 & 0 & 1
\end{array}\right]
\end{gathered}
$$

In Eq. 3.11, the Earth's rotation rate with respect to an ECI frame is represented by $\omega_{i e}$ and $t_{o}$ is the reference epoch at which the ECI and ECEF frame are co-incident (e.g. J2000 is a typical ECI realization). After transforming the body-to-inertial (i.e. ECI) DCM into the body-to-navigation (i.e. locally-level NED) DCM, the platform's traditional aircraft Euler angles are extracted using Eq. 3.12, Eq. 3.13, and Eq 3.14: where $C_{b}^{n}$ is the body to navigation DCM, and the number subscript 
corresponds to the row and column location within the DCM [40].

$$
\begin{gathered}
\phi=\operatorname{atan} 2\left(C_{b 31}^{n}, C_{b 32}^{n}\right) \\
\theta=\operatorname{acos}\left(C_{b 33}^{n}\right) \\
\psi=-\operatorname{atan} 2\left(C_{b 13}^{n}, C_{b 23}^{n}\right)
\end{gathered}
$$

\subsection{INS Error Model}

To calculate INS error-state system matrix, $F$, the derivative of each error-state model equation with respect to each error state must be taken, where the INS states can be seen in Eq. 3.15. This derivation closely follows that outlined in Groves [39]. In this section, the time derivative of the attitude, velocity, and position are described. After defining the time derivatives of the error state equations, the total system matrix and the state transition matrix (STM) is defined. The time

derivative of the attitude error can be seen in Eq. 3.16: where $\hat{C}_{b}^{i}$ is the estimated body to inertial transition matrix, and $b_{g}$ is the estimated bias on the gyroscope.

$$
\begin{gathered}
\mathbf{x}_{\mathbf{i n s}}=\left(\begin{array}{c}
\delta \Psi \\
\delta v \\
\delta r \\
b_{a} \\
b_{g}
\end{array}\right) \\
\delta \dot{\Psi}^{i}=\hat{C}_{b}^{i} b_{g}
\end{gathered}
$$

Because the velocity error is a function of the accelerometer bias, gyroscope bias, and the gravity model that is employed, it is slightly more complicated than the attitude error. The time derivative of the velocity error, $\delta \dot{V}^{i}$, can be seen in Eq. 3.17. In Eq. $3.17 \hat{C}_{b}^{i}$ is the body to inertial frame transformation matrix, $\hat{f}^{i}$ is the IMU accelerometer measure specific force in the inertial frame $\delta v / \tau$, $\delta \Psi^{i}$ is the estimated attitude error, $g$ is the estimated gravity vector for the platforms position, $r_{e s}^{e}$ is the geocentric radius at the platform position, $\hat{r}^{i}$ is the INS estimated position vector, $\delta r^{i}$ is the 
estimated position error, and $b_{a}$ is the estimated accelerometer sensor biases.

$$
\delta \dot{V}^{i}=-\left(\hat{C}_{b}^{i} \hat{f}^{i}\right) \delta \Psi^{i}+\frac{2 g}{r_{e s}^{e}} \frac{\hat{r}_{i b}^{\hat{i}}}{\left|r_{i b}^{\hat{i}^{2}}\right|}{\hat{r^{i}}}^{T} \delta r^{i}+\hat{C}_{b}^{i} b_{a}
$$

With INS mechanized in an inertial frame, the time-derivative of position is simply velocity. This means that the time-derivative of the position error in the inertial frame is the velocity error.

$$
\delta \dot{r}^{i}=\delta V^{i}
$$

Using the time derivative of each error state equation, the system matrix is defined in Eq. 3.19.

$$
F^{i}=\left[\begin{array}{ccccc}
0_{3} & 0_{3} & 0_{3} & 0_{3} & \hat{C}_{b}^{i} \\
-\left(\hat{C_{b}^{i}} \hat{f}^{i}\right) & 0_{3} & \frac{2 g}{r_{e s}^{e}} \frac{\hat{r^{i}}}{\left|\hat{r}^{2}\right|} \hat{r}^{i} & \hat{C_{b}^{i}} & 0_{3} \\
0_{3} & I_{3} & 0_{3} & 0_{3} & 0_{3} \\
0_{3} & 0_{3} & 0_{3} & 0_{3} & 0_{3} \\
0_{3} & 0_{3} & 0_{3} & 0_{3} & 0_{3}
\end{array}\right]
$$

The discrete System Transformation Matrix (STM) is then calculated using the system matrix and Eq. 3.20: where $F^{i}$ is the system matrix, and $\tau$ is the discretization interval. For this study, a third-order approximation of the STM was used as shown by the expansion in Eq. 3.21, as provided by Groves[39].

$$
\Phi=e^{F^{i} \tau}
$$

$\Phi^{i}=\left[\begin{array}{ccccc}I_{3} & O_{3} & O_{3} & O_{3} & \hat{C}_{b}^{i} \tau \\ \left(F_{21}^{i} \tau+\frac{1}{6} F_{23}^{i} F_{21}^{i} \tau^{2}\right) & \left(I_{3}+\frac{1}{2} F_{23}^{i} \tau^{2}\right) & \left(F_{23}^{i} \tau+\frac{1}{6} F_{23}^{i}{ }^{2} \tau^{3}\right) & \left(\hat{C}_{b}^{i} \tau+\frac{1}{6} F_{23}^{i} \hat{\left.C_{b}^{i} \tau^{3}\right)}\right. & \left(\frac{1}{2} F_{21}^{i} \hat{C}_{b}^{i} \tau^{2}\right) \\ \left(\frac{1}{2} F_{21}^{i} \tau^{2}\right) & \left(I_{3} \tau+\frac{1}{6} F_{23}^{i} \tau^{3}\right) & \left(I_{3}+\frac{1}{2} F_{23}^{i} \tau^{2}\right) & \left(\frac{1}{2} \hat{C}_{b}^{i} \tau^{2}\right) & \left(\frac{1}{6} F_{21}^{i} \hat{C}_{b}^{i} \tau^{3}\right) \\ 0_{3} & 0_{3} & 0_{3} & I_{3} & 0_{3} \\ 0_{3} & 0_{3} & 0_{3} & 0_{3} & I_{3}\end{array}\right]$ 


\title{
Chapter 4
}

\section{GPS INS Integration}

\author{
Parts of this chapter are reproduced from conference paper "Performance Character- \\ ization of Tightly-Coupled GNSS Precise Point Positioning Inertial Navigation within \\ a Simulation Environment" [34]
}

The integration of GPS and INS data is a well studied field and discussion of its integration can be trcked back quite far [41]. The reason the fusion of these two data sources is so prevalent, in navigation applications, tracks back to the complementary nature of the data itself. These implies that the two error sources are completely different. The INS is stable over short time intervals but the error grows unbounded if left unaided. On the other hand, the GPS errors are essentially time invariant. For GPS/INS integration there are three strategies that have been well studied; however, for aerospace applications only the first two are commonly used, so our discussion will be limited to those. The first, and simplistic to implement, is the loosely coupled method [42]. This method essentially has two distinct systems where to data is fused after the INS and GPS systems have separately calculated their respective state estimate. The tightly coupled method utilizes that raw pseudorange and carrier-phase data from the GPS receiver to estimate a single state estimate with the INS. For applications where an aircraft is either experiencing high dynamics or a GPS challenging environment, the tightly-coupled architecture has been shown to have an advantage [9], [10]. 


\subsection{Integration Methods}

This section will provide a detailed discussion of the two GPS/INS integration strategies commonly used for aerospace applications.

\subsubsection{Loosely Coupled}

The loosely coupled architecture is an integration method that utilizes three distinct sub-systems, which can be seen in Fig. 4.1. This method is also commonly called decentralized in literature.

The first sub-system is the GPS block. In this block there is a GPS receiver that collects raw pseudorange and carrier-phase data. These observables are then used to estimate the platforms position using a GPS only state estimator. If an estimate of the platforms velocity is desired that the doppler observable can also be processed within the GPS module.

The next sub-system contains the INS. This block is composed of an IMU and a navigation processor. Within the navigation processor are the algorithms to mechanize the IMU data, which can be found in section 3.3.

Finally, there is a GPS/INS kalman filter that is used to estimate the platforms total state. This. Additionally, it should be noted that the loosely-coupled system can also operate in a closed-loop manner where the INS estimated errors are fed-back to the IMU at every GPS update.

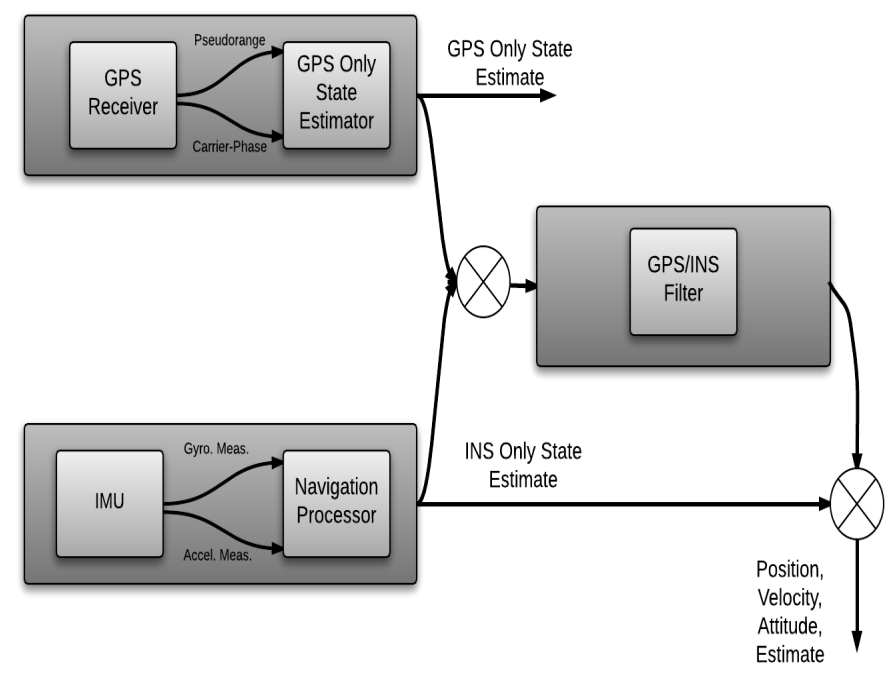

Figure 4.1: Loosely-Coupled GPS/INS 


\subsubsection{Tightly Coupled}

Now, the tightly-coupled, or centralized, integration architecture can be discussed. A schematic of the integration method can be seen in 4.2. From this diagram it should be noted that there are no longer three distinct sub-systems. Instead, difference between the GPS observables and the INS predicted observables are used as the input to the Kalman filter. Where the INS predicted measurements are calculated using the known satellite position and user position and velocity from the INS

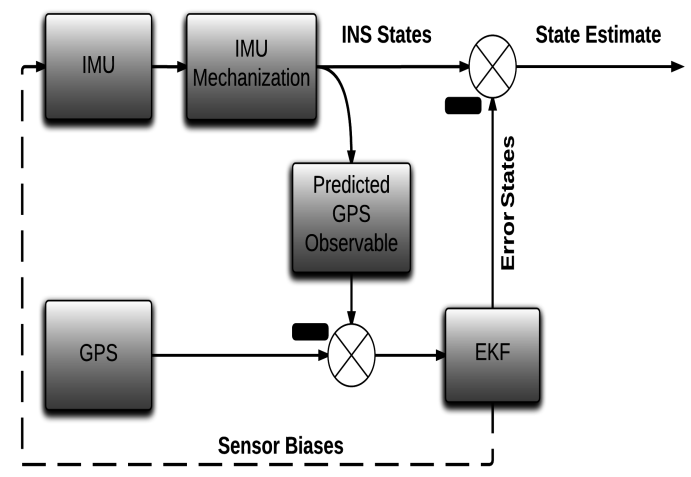

Figure 4.2: Tightly-Coupled GPS/INS

\subsubsection{Deeply Coupled}

In addition to the loosely-coupled and tightly-coupled integration stragities, there is also a deeply copuled integration stragety first introduced by Soloviev [43]. In this integration stragety, the inertial data and measurement residuals used to control the signal correlation process inside the GPS receiver [44]. This method of integration has the benefit of being able to track and reqaquire GPS observables that are much weak than nominal signal power [44].

\subsubsection{Comparison of Integration Methods}

Now that the two integration methods commonly used for aerospace navigation applications have been discussed, it is beneficial to compare the two methods. The only true difference between the integration strategies is the type of information shared between the two systems. In the looselycoupled system, the GPS computed solution is combined with the INS computed solution. On the other hand, with the tightly-coupled system the raw GPS observables are combined with the INS predicted GPS observables. With that difference in mind, it should be noted that both integration strategies will provide the platform with the ability to estimate the same states ( e.g. attitude, 
velocity, position, carrier-phase bias ). The advantages of the loosely-coupled systems come from the independence of the two measurement systems. This separation of the two filters means that each filter can be less complex, which implies less computational complexity and faster processing. Additionally, the separation of the GPS and INS makes the system robust to sensor failures. The true advantage of the tighly-coupled system becomes apparent when considering kinematic airborne applications. For these applications it is common to have abrupt attitude changes that induce poor GPS satellite geometry. With the tightly-coupled system architecture it is possible to update the filter with GPS data even if there are less than four satellites in view [39].

\subsection{Extended Kalman Filter}

Within the navigation community the Kalman filter [45] is the algorithm of choice to optimally combining multiple data sources. The Kalman filter is a recursive state space estimator that contains two broad steps: propagation and update. In this work a derivation of the Kalman filter will not shown, as it is widely available in literature [46]; however a schematic depicting the Kalman filter can be seen in Fig. 4.3 and a detailed discussion will be provided in this section.

As stated above, the Kalman filter is an optimal state estimator (optimal with respect to mean square error of the estimated states); however, the linear Kalman filter, introduced in 1960, is only optimal for linear dynamics and measurement models. One of the most common ways to extend this idea to non-linear dynamics is called the Extended Kalman Filter (EKF) [46]. The EKF uses that same data processing technique as the Kalman filter except the system is linearized about some nominal point (in our case around the output of the INS). In addition to the EKF, there is another commonly used non-linear state estimation technique known as the unscented Kalman filter (UKF) [47]. This techniuqe utilizes the unscented transformation to propagate mean and covariance. The UKF is beneficial when the system is very non-linear on the time scale of the measuremtn update; however, it has been noted in literature that this is not the case for GPS/INS integration [48]. Because of this, the EKF was selected as the filtering technique for this research.

With that in mind, Fig. 4.3 can now be described. As shown in the figure, there are two major steps associated with the Kalman filter: the prediction and the update of the estimates after the measurements. 


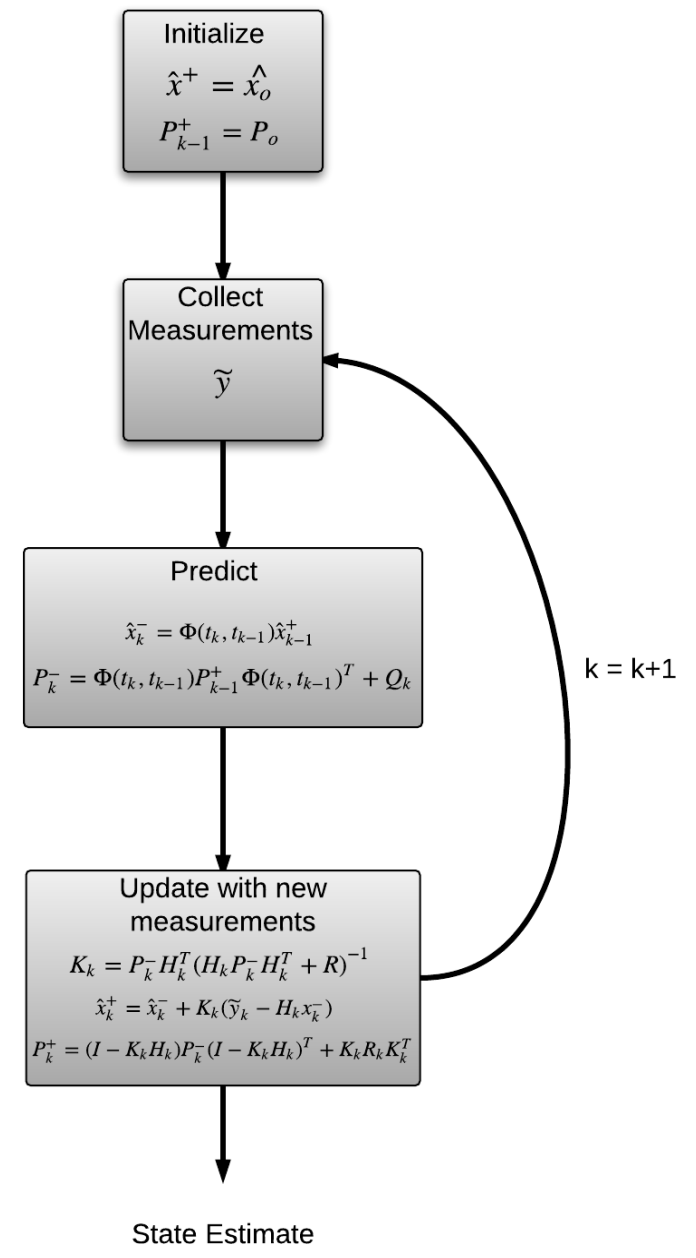

Figure 4.3: Kalman Filtering Algorithm Schematic

\subsubsection{Prediction}

The prediction step is used to propagate the state estimate, $\hat{x}$, and associated covariance matrix, $P$, using the system dynamics. The equation to propagate the state estimate can be seen in Eq. 4.1: where the is used to signify an estimated parameter, $\Phi$ is the state transition matrix (STM), the subscript $k$ or $k-1$ is used to represent the time step, and the superscript - signifies that the prediction is before the measurement update.

$$
\hat{x}_{k}^{-}=\Phi\left(t_{k}, t_{k-1}\right) \hat{x}_{k-1}^{+}
$$


The equation to propagate the covariance matrix can be seen in Eq. 4.2: where $Q_{k}$ is the process noise covariance or the amount of uncertainty in our ability to propagate the state.

$$
P_{k}^{-}=\Phi\left(t_{k}, t_{k-1}\right) P_{k-1}^{+} \Phi\left(t_{k}, t_{k-1}\right)^{T}+Q_{k}
$$

\subsubsection{Measurement Update}

Before the state estimate and covariance can updated with the new measurements, the Kalman gain must be calculated. This is because the Kalman gain is a metric to inform the filter of the relative weight that should be applied to the state estimate and the measurements. The Kalman gain can be calculated using Eq. 4.3. The $R$ in Eq. 4.3 is the measurement covariance matrix and $H$ is the measurement sensitivity matrix.

$$
K_{k}=P_{k}^{-} H_{k}^{T}\left(H_{k} P_{k}^{-} H_{k}^{T}+R\right)^{-1}
$$

After the Kalman gain has been calculated, both the state estimate and the associated covariance can be updated with the new measurements. The equations to propagate both the state estimate and the covariance can be seen in Eq. 4.4 and 4.5, respectively. In Eq. 4.4, $H_{k}$ is a matrix that is used to map the estimated states to the measurements, and $\widetilde{y}$ is the measurement vector.

$$
\begin{gathered}
\hat{x}_{k}^{+}=\hat{x}_{k}^{-}+K_{k}\left(\widetilde{y}_{k}-H_{k} x_{k}^{-}\right. \\
P_{k}^{+}=\left(I-K_{k} H_{k}\right) P_{k}^{-}\left(I-K_{k} H_{k}\right)^{T}+K_{k} R_{k} K_{k}^{T}
\end{gathered}
$$

And because the Kalman filter is a recursive algorithm, this whole process starts over again with new measurements.

\subsubsection{Assigning Process and Measurement Noise}

The Kalman filter has tree tuning matricies that must be specified for the specific application. Since the Kalman filter is known to be sensitive to tuning parameters [46], this section will detail the specific tuning for this application. 


\section{State Covariance Matrix - P}

The initial state covariance matrix, $P_{o}$ is used to define the amount of uncertienty that is assumed in the initial states. In this application, $P_{a t t}, P_{v e l}$, and $P_{p o s}$, are the amount of uncertienty in the attitude, velocity, and position states, respectively. These values were found emperically through several simulation trials. The elements $P_{a c c}$ and $P_{\text {gyro }}$ are the amount of uncertienty that is assumed in the accelerometer and gyroscope bias states, respectively. Those values are provided my the IMU manufacturer and are generally denoted as the velocity and angular random walk.

$$
P_{\text {ins }}=\left(\begin{array}{ccccc}
P_{\text {att }} & & & & \\
& & & & \\
& P_{\text {vel }} & & & \\
& & P_{\text {pos }} & & \\
& & P_{\text {acc }} & \\
& & & P_{\text {gyro }}
\end{array}\right)
$$

In addition to the INS states, the amount of uncertienty in the GPS states (the carrier-phase bias for each satellite in view), is also constructed as a diagonal matrix with 25 meters in every diagonal term. The construction of the total inital state covariance matrix is shown in Eq. 4.10.

$$
P_{o}=\left(\begin{array}{cc}
P_{i n s} & 0 \\
0 & P_{g p s}
\end{array}\right)
$$

\section{Measurement Noise Covariance Matrix - $\mathbf{R}$}

The measurement noise covariance matrix, $R$, is used to account for several unaccounted for GPS errors (i.e. multipaht, thermal noise, variations in the atmosphere, and satelltie clock noise). This matrix is usually diagonal unless the pseudorange measurements are carrier-smoothed [39]. In this research the $R$ matrix is a function of the elveation angle of the satellite. This can be seen in Eq. 4.8: where $e l^{j}$ is used to denote the elevation anlge between the receiver and the $j^{t} h$ satellite.

$$
R_{k}(j, j)=\frac{1}{\sin \left(e l^{j}\right)} R_{k-1}(j, j)
$$

\section{Process Noise Covariance Matrix - Q}

The process noise covariance matrix, $Q$, is used to describes how much the state is expected to vary over the interval of one time step due to the effect of the process noise [36].In this case, $Q_{g p s}$ is matrix composed of zeros where the size is dependant upon the number of satellites in view. $Q_{\text {ins }}$ 
is a matrix composed of the uncertinty in the INS states, and can be found by looking at the IMU error specifications.

$Q_{a t t}$ and $Q_{v e l}$ are related to the angular random ralk (ARW) and velocity random walk (VRW) associated with the IMU used, respectively. Additionally, the $Q_{a c c}$ and $Q_{g y r o}$ are related to the gyroscope in-run bias and the accelerometer in-run bias associated with the IMU used, respectively.

$$
\begin{aligned}
& Q_{\text {ins }}=\left(\begin{array}{ccccc}
Q_{\text {att }} & & & & \\
& Q_{\text {vel }} & & & \\
& & Q_{\text {pos }} & & \\
& & & Q_{a c c} & \\
& & & & Q_{\text {gyro }}
\end{array}\right) \\
& Q=\left(\begin{array}{cc}
Q_{i n s} & 0 \\
0 & Q_{g p s}
\end{array}\right)
\end{aligned}
$$

\subsection{Tightly Coupled Error State Extended Kalman Filter}

With the information provided in the last three chapters, the data fusion architecture selected for this research can be discussed. Due to the dynamic nature of the platform that is being used, a tightly-coupled error-state extended Kalman filter is adopted from Groves[39]. A schematic of the tightly coupled PPP/INS integration architecture can be seen in Figure 4.2. Using the difference between the GPS observables and the INS predicted observables, the Extended-Kalman filter is used to estimate the INS solution errors with the state vector as shown in Eq. 4.11: where $\delta \Psi$ is the estimated attitude error, $\delta v$ is the estimated velocity error, $\delta r$ is the estimated position error, $b_{a}$ is the estimated accelerometer sensor biases, $b_{g}$ is the estimated gyroscope sensor biases, $\delta t_{u}$ is the estimated receiver clock bias, $T_{w}$ is the estimated residual tropospheric delay along the zenith 
direction, and $N$ is the estimated phase bias for each satellite in view.

$$
\mathbf{x}=\left(\begin{array}{c}
\delta \Psi \\
\delta v \\
\delta r \\
b_{a} \\
b_{g} \\
\delta t_{u} \\
T_{w} \\
N_{1} \\
\vdots \\
N_{j}
\end{array}\right)
$$

It can also be seen in Figure 4.2 that the estimated sensor biases are fed-back to correct the raw IMU measurements. Feedback is done at every time that a GPS update data is collected in a closed-loop manner. For the position and velocity estimated error states $\delta v$ and $\delta r$ are used to correct INS by subtracting them off of the INS estimated position and velocity.

\subsection{PPP Measurement Sensitivity Matrix}

The measurement sensitiviy matrix, $H$, is used to map the estimated states to the measured values. Becuause of this, the $H$ matrix must be defined specifically for each application. This section will detail the measurement sensitivity matrix for a PPP/INS filter.

The $H$ matrix can be decomosed into two sections: the pseudorange section and the carrier-phase section. The measurement sensitivity matrix for the pseudorange measurements is seen in Eq. 4.12: where $u_{i}$ is the unit vector between the $i^{t h}$ satellite and the receiver, and $m_{i}$ is the elevation angle dependant mapping for the troposphere scalling.

$$
H_{\text {pseudorange }}=\left(\begin{array}{cccccc}
0_{1 x 6} & u_{i} & 0_{1 x 6} & 1 & m_{i} & 0_{1 x n} \\
\vdots & & n-2 & & & \vdots \\
0_{1 x 6} & u_{i} & 0_{1 x 6} & 1 & m_{i} & 0_{1 x n}
\end{array}\right)
$$

The measurement sensitivity matrix for the carrier-phase data is show in Eq. 4.13: where where $u_{i}$ is the unit vector between the $i^{t h}$ satellite and the receiver, $m_{i}$ is the elevation angle dependant 
mapping for the troposphere scalling, and $e_{i}$ is the $i^{\text {th }}$ row of an idenity matrix.

$$
H_{\text {carrier }_{p} \text { hase }}=\left(\begin{array}{cccccc}
0_{1 x 6} & u_{i} & 0_{1 x 6} & 1 & m_{i} & e_{i} \\
\vdots & & n-2 & & & \vdots \\
0_{1 x 6} & u_{i} & 0_{1 x 6} & 1 & m_{i} & e_{i}
\end{array}\right)
$$

The total $H$ matrix can be constructed as shown in Eq. 4.14: where the total dimensions of the matrix are $2 n x N+n$. Where $n$ is the number of satellites in view at the epoch and $N$ is the number of non-bias parameters (i.e. attitude, velocity, position).

$$
H_{\text {total }}=\left(\begin{array}{c}
H_{\text {pseudorange }} \\
H_{\text {carrier-phase }}
\end{array}\right)
$$

\subsection{IMU to GPS Lever Arm}

The above INS mechanization provides estimates of the position and velocity located at the center of the IMU. In order to combine with GNSS measurements, the INS solution must be transposed to the GNSS antenna location. This can be done using the estimated platform attitude $\hat{C}_{b}^{i}$ and knowledge of the lever arm from the IMU to the GNSS antenna, $L_{b}$, represented in the platforms North, East, Down (NED) body-axis.

$$
r_{k \mid k-1}^{i, G P S A n t .}=r_{k \mid k-1}^{i, I M U}+\hat{C}_{b}^{i} L_{b}
$$

Likewise the velocity can be transposed further taking into consideration the rotation of the body-frame.

$$
v_{k \mid k-1}^{i, G P S A n t .}=v_{k \mid k-1}^{i, I M U}+\hat{C}_{b}^{i} \Omega_{i b}^{b} L_{b}
$$

where $\Omega_{i b}^{b}$ is the skew-symmetric matrix of the IMU measured angular rate that has been calibrated by the estimated gyroscope biases. The operation is performed upon each GNSS measurement update, and reversed after closed-loop feedback correction has been applied, in order to resume INS integration about the location of the IMU. 


\subsection{GNSS/IMU Measurement Time-Alignment}

Most commercially available high-accuracy GNSS/IMU systems provide IMU measurements precisely stamped to the GPS time- scale, however, the IMU measurements are typically not scheduled to be precisely aligned with the GPS measurement epochs. To ensure time-alignment in the RTGx implementation, the IMU data was used to predict the navigation state to an epoch just past the GNSS observation epoch. This prediction was then used to linearly interpolate the navigation states back to the time of the GNSS measurements. Likewise, upon each GNSS update, an error-state transformation matrix, that provides the mapping of the INS error-states between the INS timestep (that is just past the GNSS measurement epoch), and the exact GNSS measurement epoch was derived. Prior to the GNSS update, the inverse of this transformation was used to down-date the predicted INS error-states to the GNSS measurement epochs. After the update was completed, this transformation was used to keep the INS error states consistent with the INS time-tags. 


\section{Chapter 5}

\section{Simulation Results}

Parts of this chapter are reproduced from conference paper "Performance Characterization of Tightly-Coupled GNSS Precise Point Positioning Inertial Navigation within a Simulation Environment" [34]

\subsection{Simulation Overview}

Using the models described in the previous chapters and a commercially available GPS constellation simulation toolbox, SatNav-3.04 Toolbox[49], a simulation environment was developed to generate raw IMU and GPS data. The simulation environment architecture can be seen in Figure 5.1. This section will provide an overview of the simulation environment. First, we will discuss the inputs to the simulation, next we will provide an overview of the GPS data generation, and finally we will discuss the generation of the IMU data.

\subsubsection{Simulation Inputs}

Before the generation of GPS and IMU data, several user-defined inputs are selected. One such input is the defined flight path. For this study, four flight paths of varying dynamics were designed to replicate typical data collection flights for NASA's UAVSAR airborne radar platform. As an example, two of the flight paths are shown in Figure 5.2 and Figure 5.3. Other inputs that must be defined are the origin (e.g Lat., Lon., Height) of the flight and the time that the flight occurs. These parameters are randomized for every simulation run so that the generated data will have differing satellite geometry and atmospheric effects. In addition, the magnitude of the GPS error sources were selected before each simulation trial (e.g., multipath intensity, troposphere, and thermal 


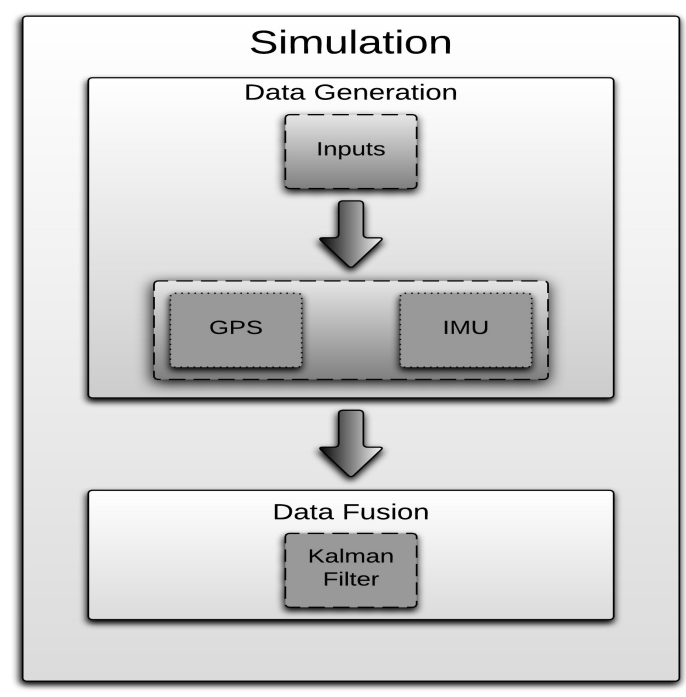

Figure 5.1: Flowchart Depicting the Simulation Environment

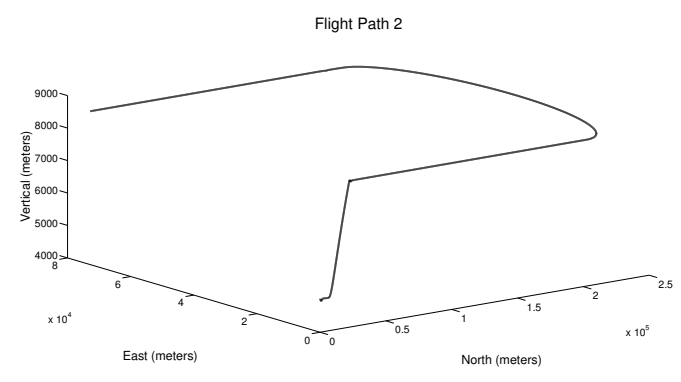

Figure 5.2: Flight Path 2

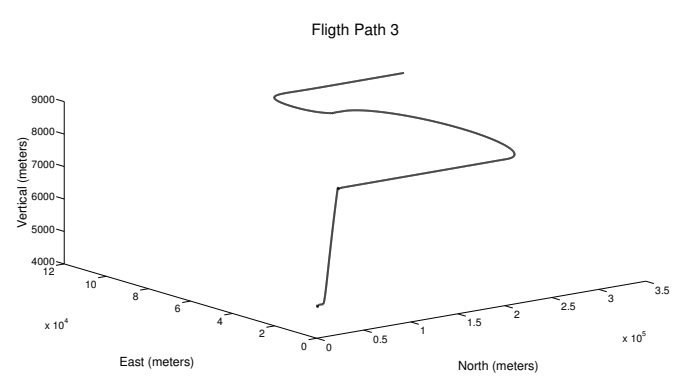

Figure 5.3: Flight Path 3

noise). Finally, one of the four IMUs that were modeled for this study is selected for each run.

\subsubsection{GPS Data Generation}

Using the SatNav-3.04 Toolbox, dual-frequency pseudorange and carrier-phase observables are generated at $10 \mathrm{~Hz}$ over the specified flight path. For this study, modifications were made that are pertinent to common aircraft positioning error-sources. For example, the GPS simulator was modified to include attitude dependent satellite visibility masking and carrier-phase breaks. Figures 5.4 and 5.5 are included to depict the attitude dependent satellite masking. That is, when a satellite is obscured or nearly obscured due to a change in platform attitude, it is masked from view and the the potential of a carrier-phase breaks is increased. Figure 5.6 and Figure 5.7 are included to depict an example of carrier-phase breaks being added into the GPS data.

This simulation also includes an orbit and clock error model. The modeled errors were determined by differing JPL's International GNSS Service (IGS) submission with European Center for Orbit 

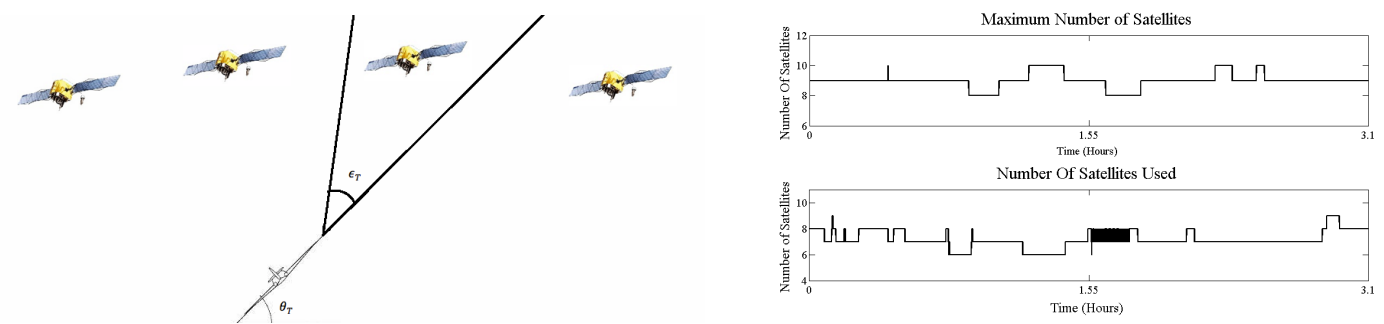

Figure 5.4: Attitude Dependent Satellite Mask- Figure 5.5: Number of Satellites in View for ing Flight

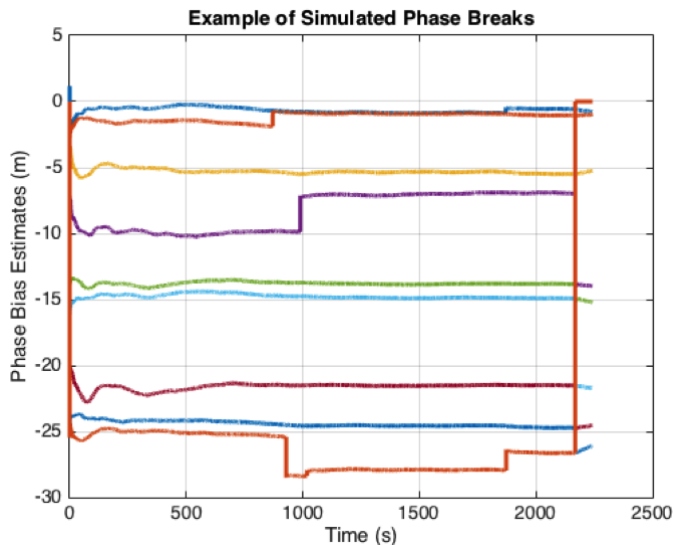

Figure 5.6: Phase Breaks for an Example Flight

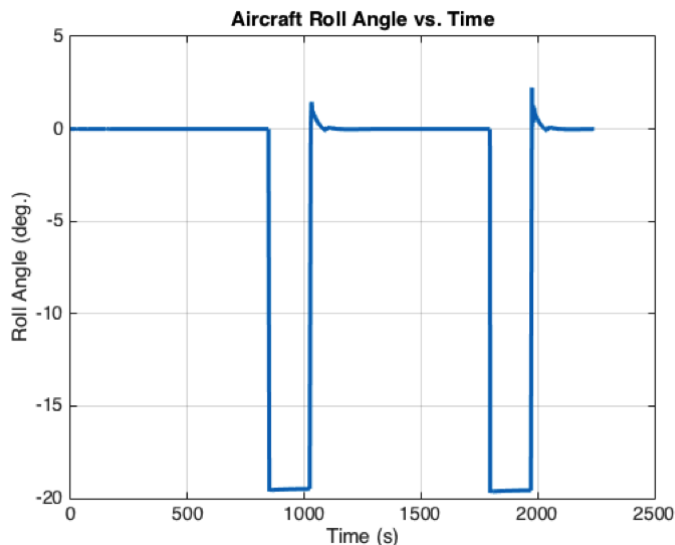

Figure 5.7: Roll for an Example Flight

Determination (CODE) submission. The error between the satellite ephemeris products are resolved in the radial, in-track, and cross-track (RIC) components. After transforming the error into RIC components, it is fit using a multi-sinusodial model. This process was completed for each satellite. The modeled satellite clock bias can be seen in Fig. 5.8.

\subsubsection{IMU Data Generation}

To generate IMU data, a commercially available, tactical grade IMU was selected to model. The IMU was modeled with a turn-on bias, a random walk component, and white noise. The modeled IMU was scaled, with respect to Table 1 , to simulated four IMUs of varying quality. The allan deviation of the four accelerometers and four gyroscopes along the $\mathrm{x}$-axis for twelve hour of data can be seen in Figure 5.9.

\subsubsection{Simulation Simplifications}

It should be noted that the simulation environment makes several simplifying assumptions. These include perfect knowledge of ECI to ECEF transformation, neglect relativistic effects (i.e. from the 


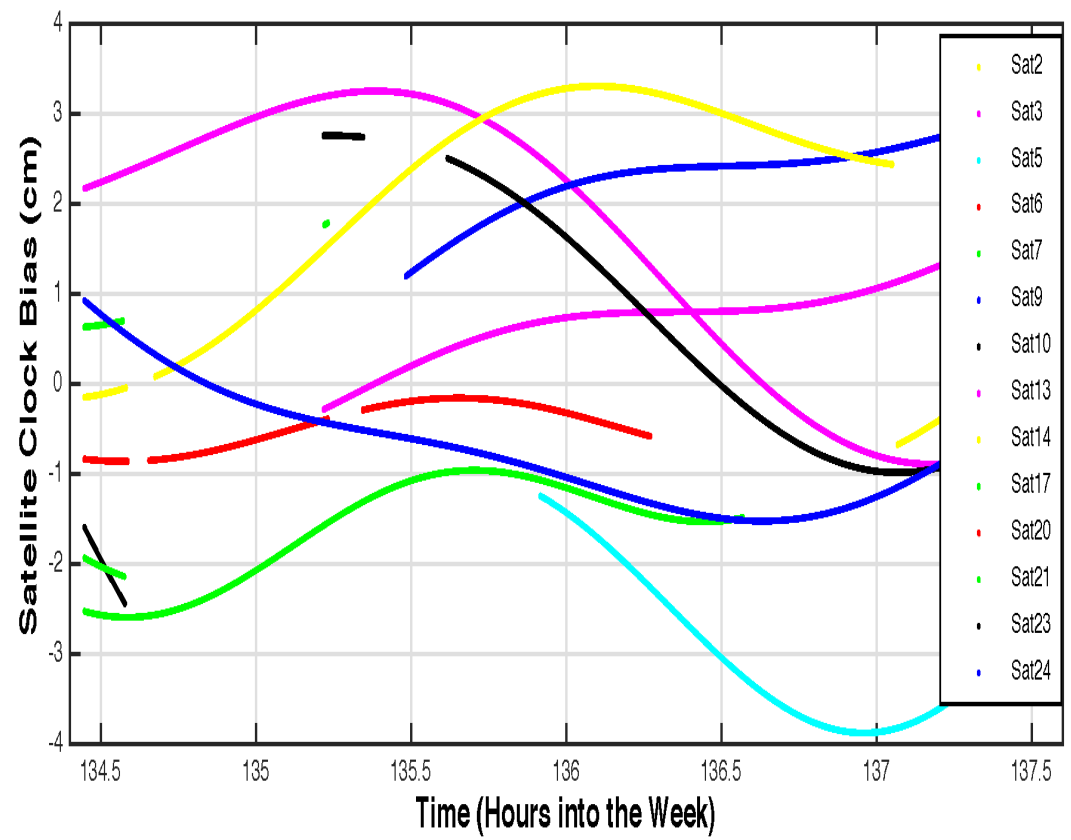

Figure 5.8: Error Between Broadcast Products of IGS and CODE Products
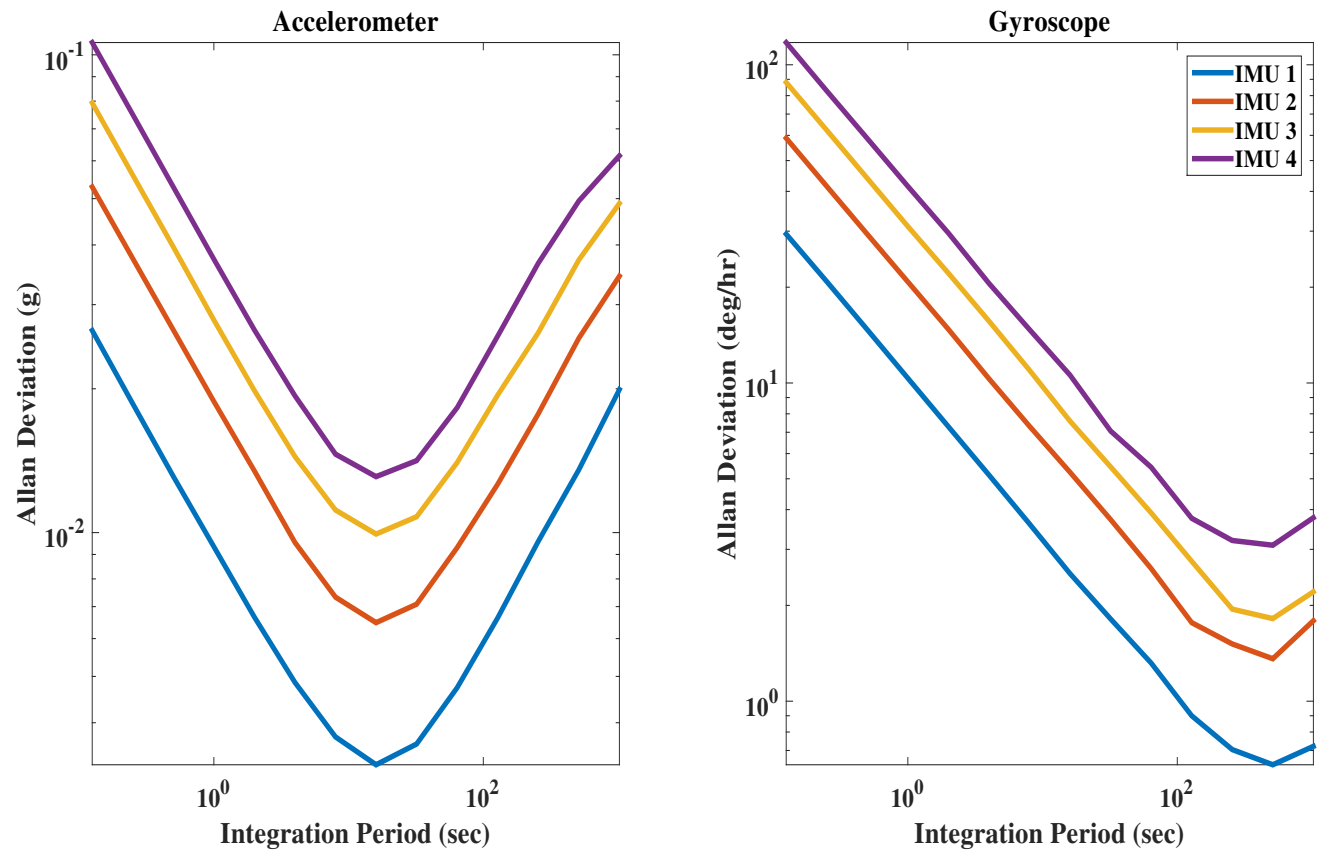

Figure 5.9: Allan Deviation for Accelerometer and Gyroscope

GPS broadcast correction), neglect of receiver and satellite antenna phase center variation, neglect of carrier-phase wind-up, and neglect of lever arm of GPS transmiting antenna. 


\subsection{Monte Carlo Design}

To generate the results shown in the next section, a Monte Carlo style design was implemented. Each simulation trail was initialized at random across the distributions specified in Table 1. One hundred sets of data were generated. GPS and IMU data were generated for every flight at ten and two hundred hertz, respectively. The one hundred data sets were run through a PPP only filter and a tightly-coupled PPP/INS filter to characterize the performance increase by including INS.

Table 5.1: Simulation Parameters

\begin{tabular}{|c|c|c|}
\hline Error-Sources & Model Parameters & Notes \\
\hline Accelerometer & $\begin{array}{l}\text { In-run Bias } \sigma=1 m g, V R W= \\
0.2 \frac{m / s}{\sqrt{h r}}\end{array}$ & $\begin{array}{l}\text { Scaled Honeywell HG1700AG72 } \\
\mathrm{SF}=\left(1, \frac{1}{50}, \frac{1}{200}, \frac{1}{400}\right)\end{array}$ \\
\hline Gyroscope & $\begin{array}{l}\text { In-run Bias } \sigma=9.6 e^{-6 \frac{\mathrm{rad}}{\mathrm{sec}}} \\
A R W=0.2 \frac{\mathrm{deg}}{\sqrt{h r}}\end{array}$ & $\begin{array}{l}\text { Scaled Honeywell HG1700AG72 } \\
\mathrm{SF}=\left(1, \frac{1}{50}, \frac{1}{200}, \frac{1}{400}\right)\end{array}$ \\
\hline Thermal Noise & $\sigma_{\rho}=0.32 m, \sigma_{\phi}=0.16 \lambda$ & $\begin{array}{l}\text { linear scale factor randomly se- } \\
\text { lected between }[0,1]\end{array}$ \\
\hline Multipath & 1.0 intensity: $\sigma=0.4 m, \tau=15 \mathrm{sec}$ & $\begin{array}{l}\text { linear scale factor randomly se- } \\
\text { lected between }[0,2]\end{array}$ \\
\hline Tropospheric Delay & $\begin{array}{l}\text { Percent of error assumed handled } \\
\text { by broadcast correction }\end{array}$ & $\begin{array}{l}\text { Modified Hopfield with linear } \\
\text { scale factor randomly selected be- } \\
\text { tween }[0,1.5]\end{array}$ \\
\hline Ionospheric Delay & $\begin{array}{l}\text { First order ionospheric effects mit- } \\
\text { igated with dual-frequency }\end{array}$ & $\begin{array}{l}\text { linear scale factor randomly se- } \\
\text { lected between }[0.7,1]\end{array}$ \\
\hline Receiver Clock Bias & Initial Bias $\sigma=30 n s, \delta \tau_{b}=100 n s$ & Tuneable \\
\hline Phase Ambiguity & $\begin{array}{l}\text { Random initialization and phase } \\
\text { breaks correlated with UAV atti- } \\
\text { tude }\end{array}$ & likelihood varied from $[0.008,0.02]$ \\
\hline Orbits & Orbits $\sigma=5 \mathrm{~cm}$ & $\begin{array}{l}\text { Description provided in section } \\
\text { III.B }\end{array}$ \\
\hline
\end{tabular}

\subsection{Results}

\subsubsection{Absolute Positioning Performance}

To quantify the potential benefits of INS inclusion for UAV navigation in scenarios that typically degrade GPS performance, a sensitivity analysis of positioning performance was conducted under varying conditions. The metric used to quantify the error for the first nine figures in this section is Root Mean Square (RMS). The equation to calculate RMS can be seen in Eq. 5.1: where $n$ is the 


\begin{tabular}{|c|c|c|c|}
\hline & $\begin{array}{c}\text { East } \\
(\mathrm{cm})\end{array}$ & $\begin{array}{c}\text { North } \\
(\mathrm{cm})\end{array}$ & $\begin{array}{c}\text { Vertical } \\
(\mathrm{cm})\end{array}$ \\
\hline Min & 4.08 & 4.89 & 16.20 \\
\hline Max & 198.07 & 259.77 & 407.50 \\
\hline Mean & 62.85 & 65.25 & 146.52 \\
\hline Median & 50.49 & 55.07 & 137.39 \\
\hline Std & 44.71 & 46.76 & 78.23 \\
\hline
\end{tabular}

Table 5.2: PPP Only Statistics (Simulation)

number of data points, $X$ is the actual value of the data, and $\hat{X}$ is the estimated value of the data.

$$
X_{R M S}=\sqrt{\sum_{i=1}^{n} \frac{1}{n}\left(X^{2}(i)-\hat{X}^{2}(i)\right)}
$$

The median of the RMS positioning error for the one hundred flight can be seen in Fig. 5.10. From Fig. 5.10 it should be noted that the PPP/INS filter outperformed PPP only filter in east, north and vertical directions, with the most notable improvement being in the vertical direction. The statistics for the one hundred flights can be seen in Table 2, Table 3 and Table 4. The next eight figures

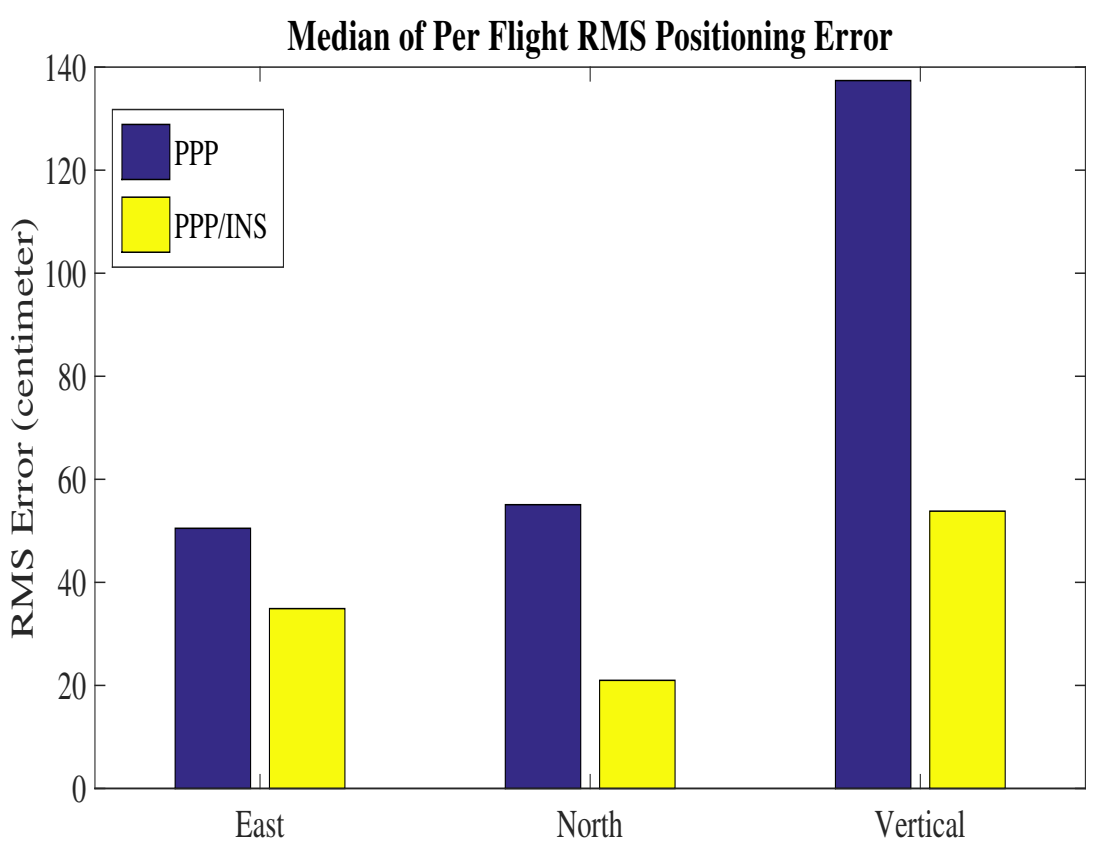

Figure 5.10: Median of Per Flight RMS Error

in this section depict the reduction in RMS error through the inclusion of INS. The reduction in error was calculated by taking the difference of the positioning output for the PPP only filter and the PPP/INS filter. Figure 5.11 shows the median of the per flight RMS error reduction due to the 


\begin{tabular}{|c|c|c|c|}
\hline & $\begin{array}{c}\text { East } \\
(\mathrm{cm})\end{array}$ & $\begin{array}{c}\text { North } \\
(\mathrm{cm})\end{array}$ & $\begin{array}{c}\text { Vertical } \\
(\mathrm{cm})\end{array}$ \\
\hline Min & 5.65 & 2.94 & 17.15 \\
\hline Max & 137.36 & 121.80 & 310.95 \\
\hline Mean & 37.88 & 25.30 & 70.55 \\
\hline Median & 34.90 & 20.99 & 53.82 \\
\hline Std & 24.23 & 19.38 & 55.81 \\
\hline
\end{tabular}

Table 5.3: PPP/INS Statistics (Simulation)

\begin{tabular}{|c|c|c|c|}
\hline & $\begin{array}{c}\text { East } \\
(\mathrm{cm})\end{array}$ & $\begin{array}{c}\text { North } \\
(\mathrm{cm})\end{array}$ & $\begin{array}{c}\text { Vertical } \\
(\mathrm{cm})\end{array}$ \\
\hline Min & -55.42 & -64.12 & -206.92 \\
\hline Max & 158.02 & 221.40 & 343.94 \\
\hline Mean & 24.96 & 39.93 & 75.97 \\
\hline Median & 16.94 & 31.76 & 69.62 \\
\hline Std & 32.65 & 38.75 & 85.31 \\
\hline
\end{tabular}

Table 5.4: (PPP - PPP/INS) Statistics (Simulation)

inclusion of INS. From Fig. 5.11 it can be seen that the error reduction in the East, 17 centimeters, and North, 32 centimeters, directions are similar. The error reduction is the vertical direction is the most notable at 70 centimeters. Figure 5.12 depicts the RMS error reduction distribution in the east, north and vertical directions for the 100 trials. This was done by calculating the cumulative distribution function (CDF) of the RMS positioning error reduction for all of the simulated flight due to INS. From 5.12 it should be noted that the median error is as shown in Fig. 5.11; however, there are situations where the inclusion of INS can reduce the vertical positioning error by as much as 200 centimeters. However, there are times where the CDF is negative, meaning that the PPP-only filter outperformed the PPP/INS filter. For the cases in which PPP-only outperformance PPP/INS it is expected that that multiple satellite loss of lock or phase breaks may have occurred early in the filter run, before the phase biases, positioning, residual troposphere delay, and IMU sensor biases converged, leaving the PPP/INS solution to "hold on" to a poor solution for a longer period than the PPP-only filter; however these flights need further review. Now that the median RMS error reduction has been discussed, it is of interest to discuss the reduction of the RMS error due to INS with respect to the input parameters (e.g., flight path, IMU selection and GPS error sources). Figure 5.13 shows the median RMS error reduction in the east, north and vertical directions with respect to the flight path selected. The four flight paths were designed to have increasing dynamics (i.e., flight path one is the most docile and flight path four has the highest dynamics). Figure 5.13 does not show a definitive trend in positioning improvement as path dynamics increase. The likely reason for this lack of trend is that the dynamics did not vary enough between paths. Figure 5.14 shows the 


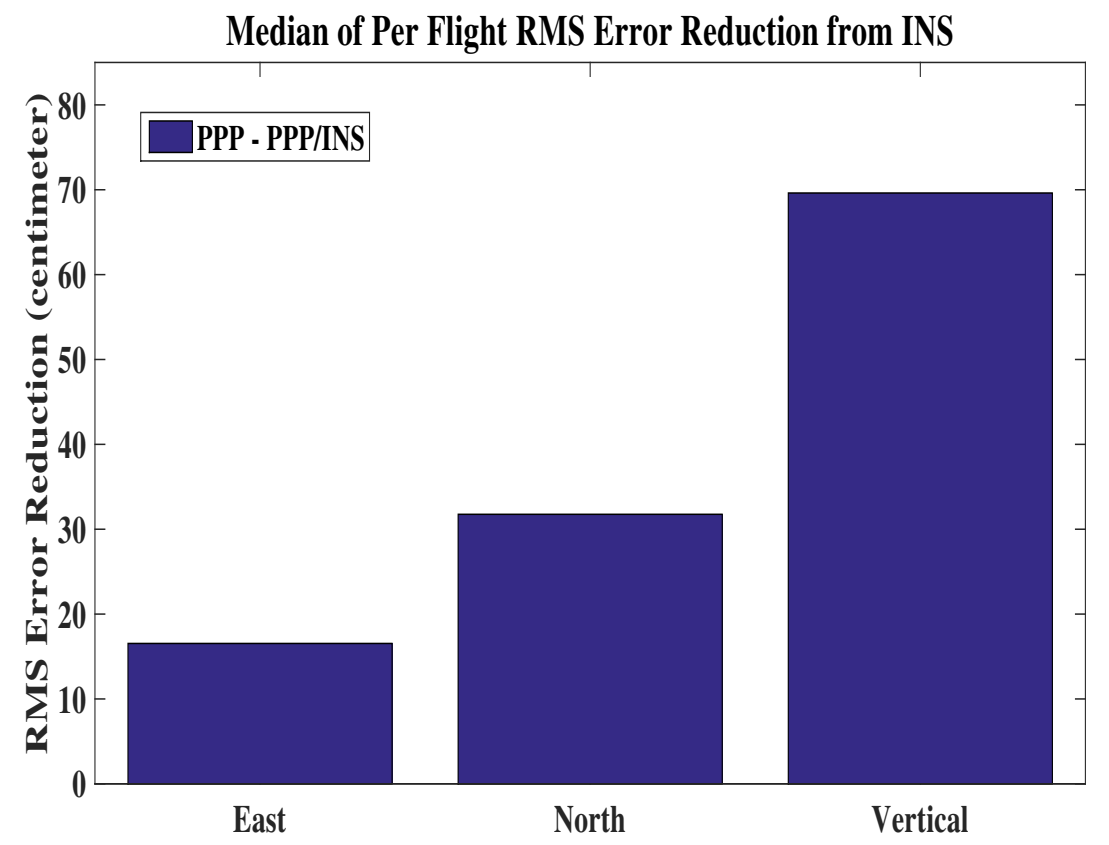

Figure 5.11: Median of Per Flight RMS Error Reduction from INS
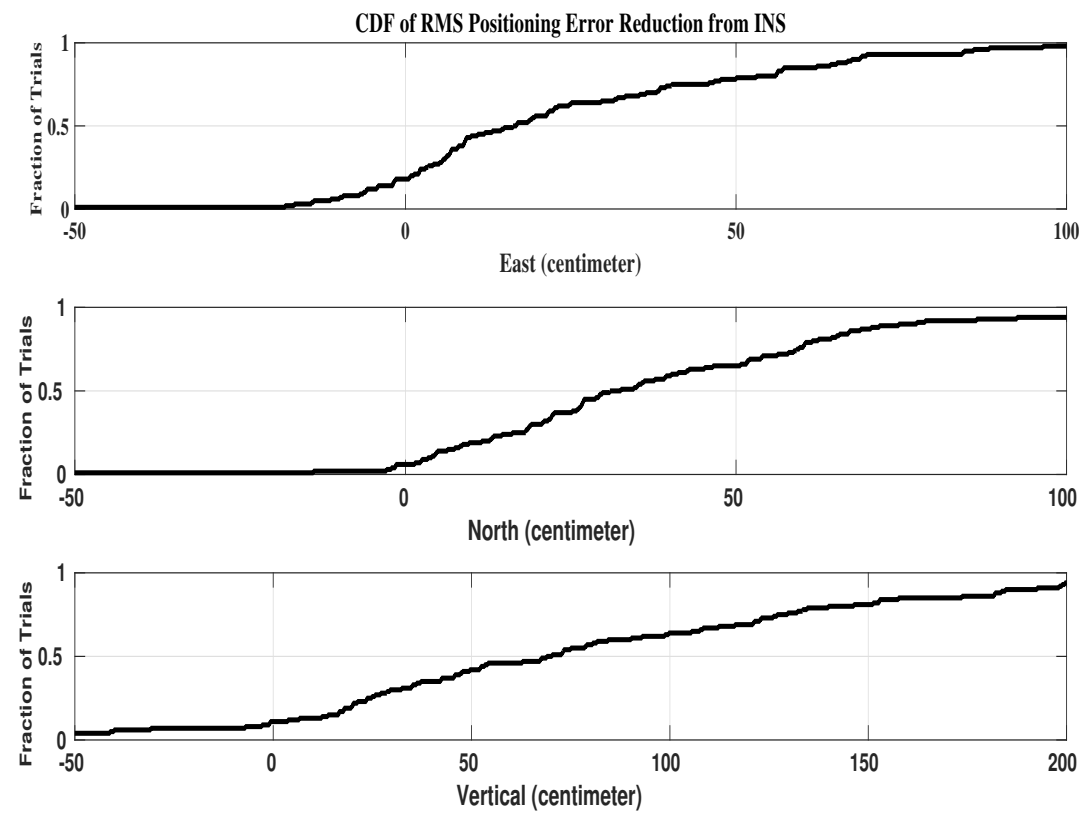

Figure 5.12: CDF of RMS Positioning Error Reduction form INS

median RMS error reduction in the east, north and vertical directions with respect to the number of phase breaks during a flight. From Fig. 5.14 it should be noted that there is a clear correlation between the number of phase breaks and the RMS positioning error reduction due to INS. 


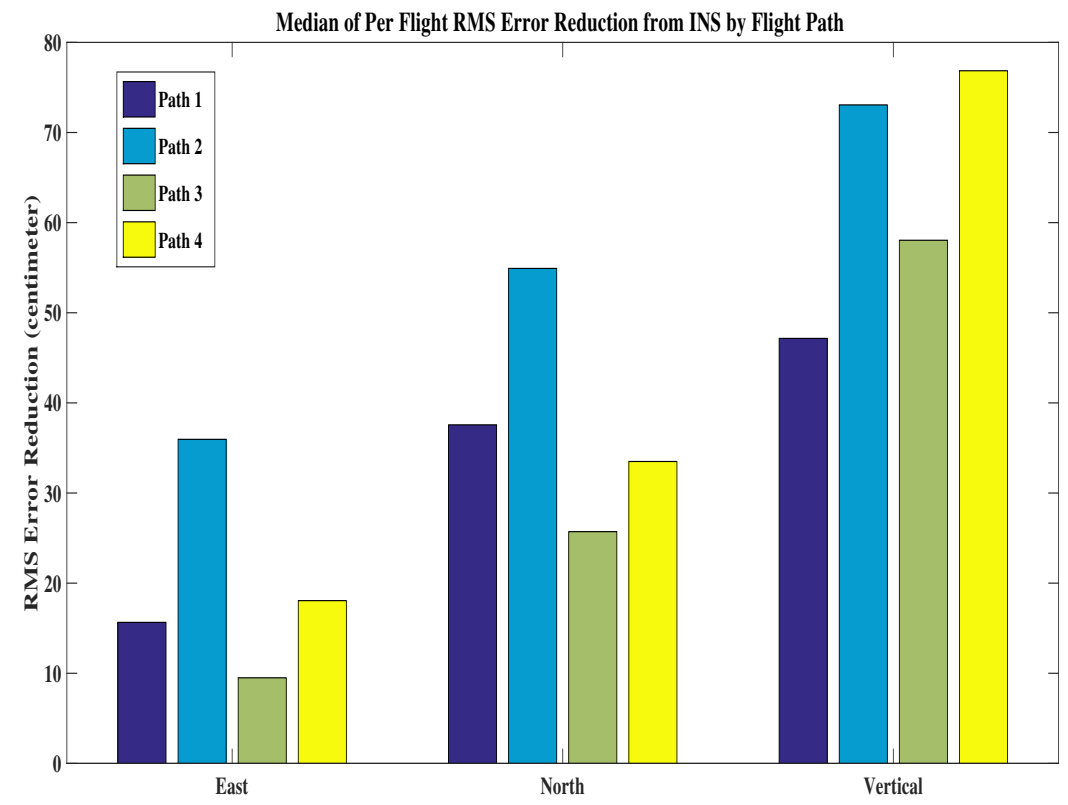

Figure 5.13: Median of Per Flight RMS Error Reduction from INS by Flight Path

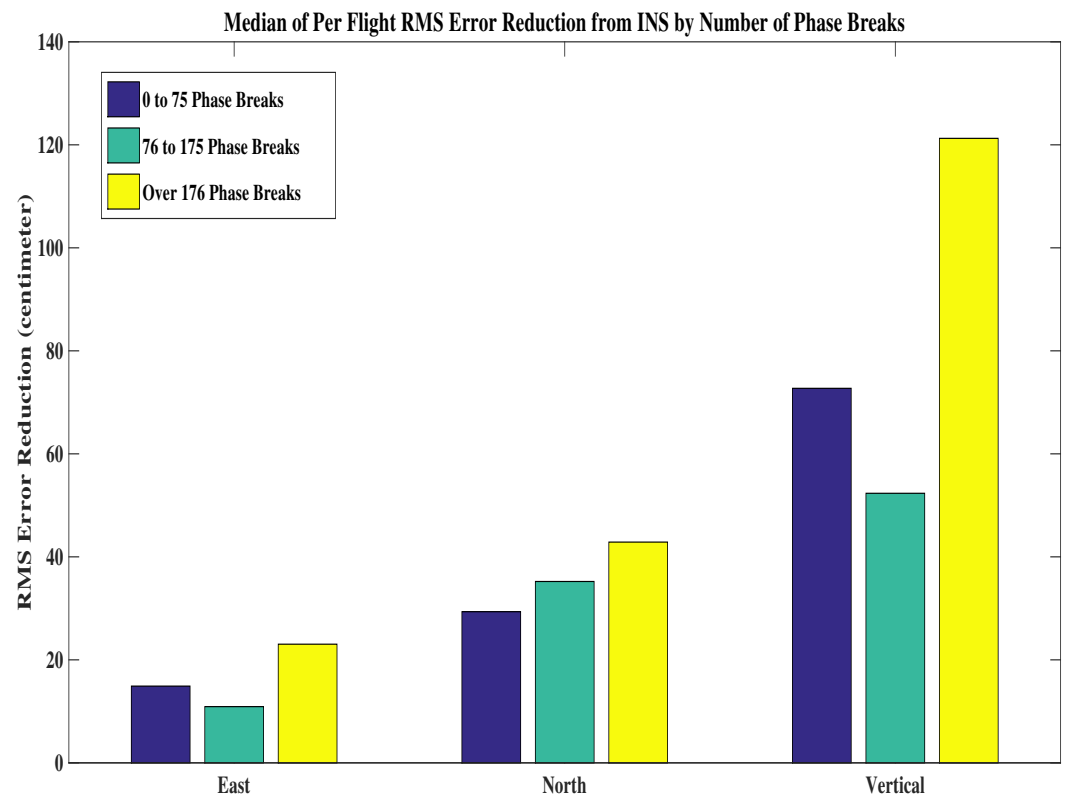

Figure 5.14: Median of Per Flight RMS Error Reduction from INS by Number of Phase Breaks

Figure 5.15 shows that median of the per flight RMS error reduction from INS by IMU type. As all of the IMUs for this study were modeled based upon error characteristics for commercially available tactical grade IMUs with similar error characteristics (e.g., all IMUs were scaled from 
IMU 1), there is not a definitive trend in positioning improvement. Figure 5.16 depicts the RMS

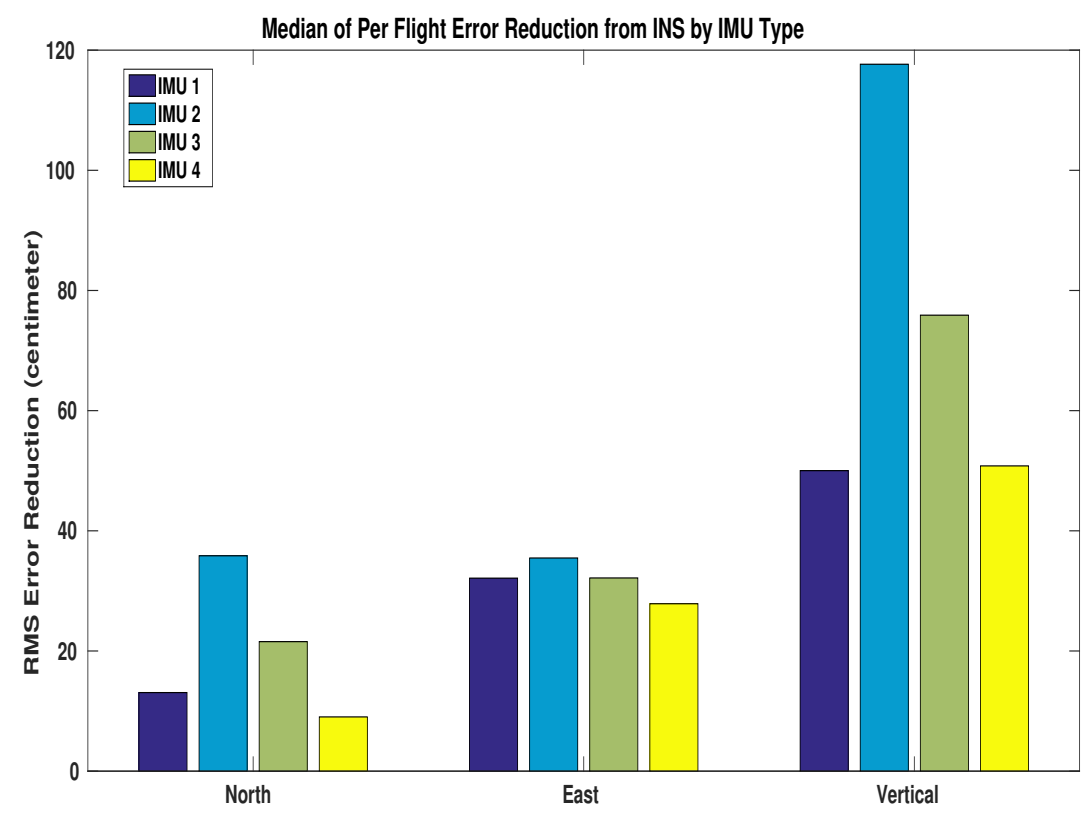

Figure 5.15: Median of Per Flight RMS Error Reduction from INS by IMU

positioning error reduction due to INS with respect to the magnitude of the troposphere. Where the troposphere is modeled using the Modified Hopfield model and scaled at random, for each simulation trial, with respect to Table 1. From Fig. 5.16 a clear trend is shown, which is that the magnitude of the RMS error reduction is dependent upon the magnitude of the troposphere. The dependence of the RMS positioning error reduction due to INS as a function of multipath can be seen in Figure 5.17. Multipath is modeled as a Gauss-Markov process with a standard deviation of 0.4 meters and a time constant of 15 seconds, as described in Table 1 . This error source is linearly scaled for each simulation trial.

\subsubsection{Sensitivity to PPP Product Quality}

Figure 5.18 depicts the benefit of including INS as the quality of the PPP products is reduced. That is, for this analysis, the GPS data was regenerated in the same manner as previously describe; however, the magnitude of the satellite error was randomly selected from four magnitude (e.g., Product $1=0.5 \mathrm{~cm}$, Product $2=10 \mathrm{~cm}$, Product $3=20 \mathrm{~cm}$, and Product $4=30 \mathrm{~cm}$ ). 


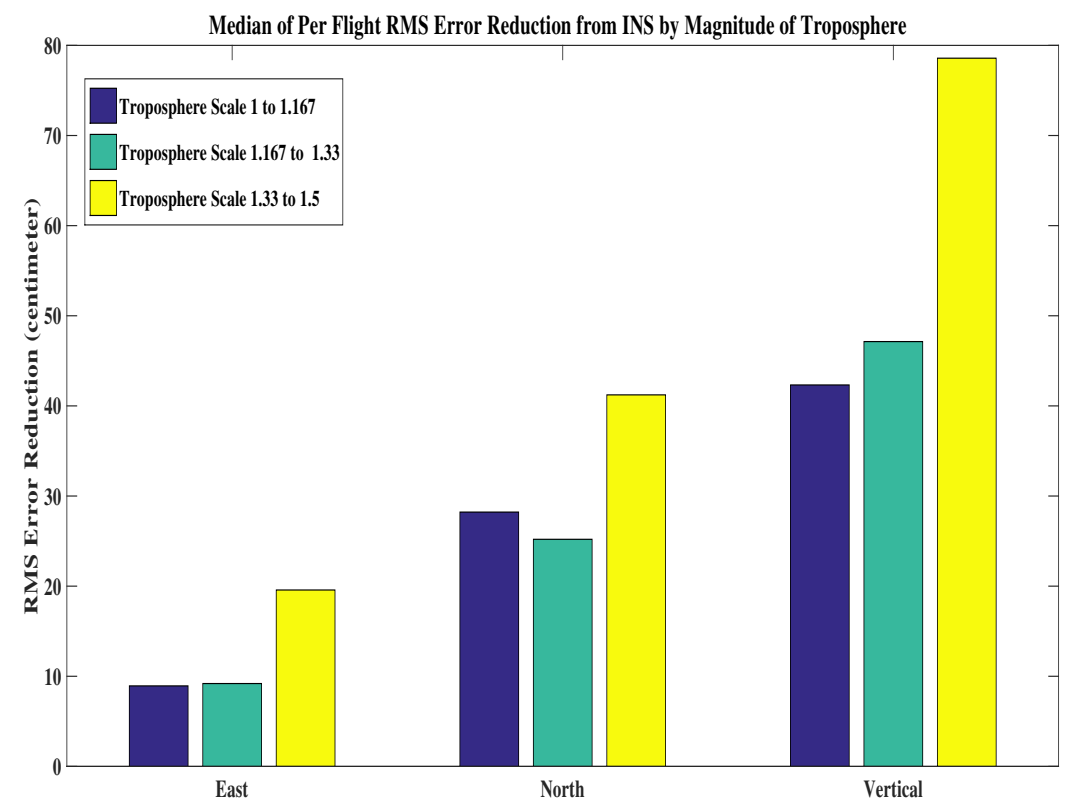

Figure 5.16: Median of Per Flight RMS Error Reduction from INS by Magnitude of Troposphere

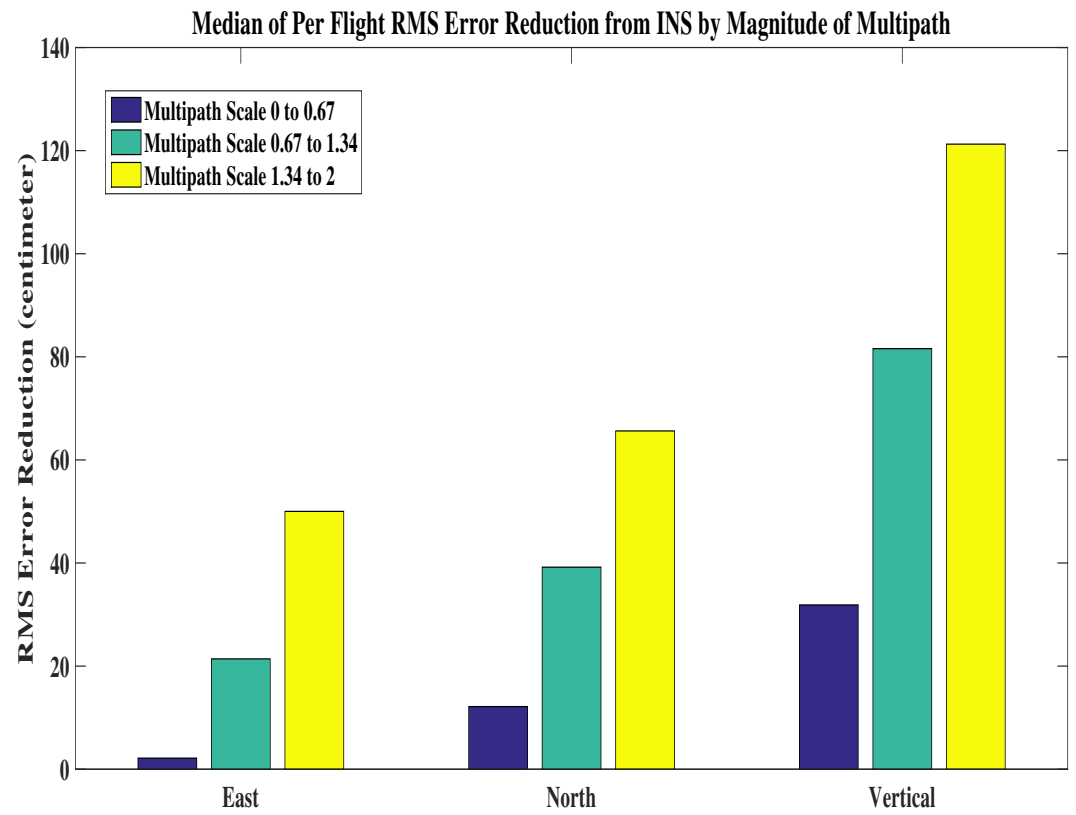

Figure 5.17: Median of Per Flight RMS Error Reduction from INS by Magnitude of Multipath

\subsubsection{Attitude Estimation Performance}

An implicit benefit of PPP/INS over single antenna PPP-only is that platform attitude is estimated in the filter. Figure 5.19 shows the typical attitude estimation performance of a single simulation 


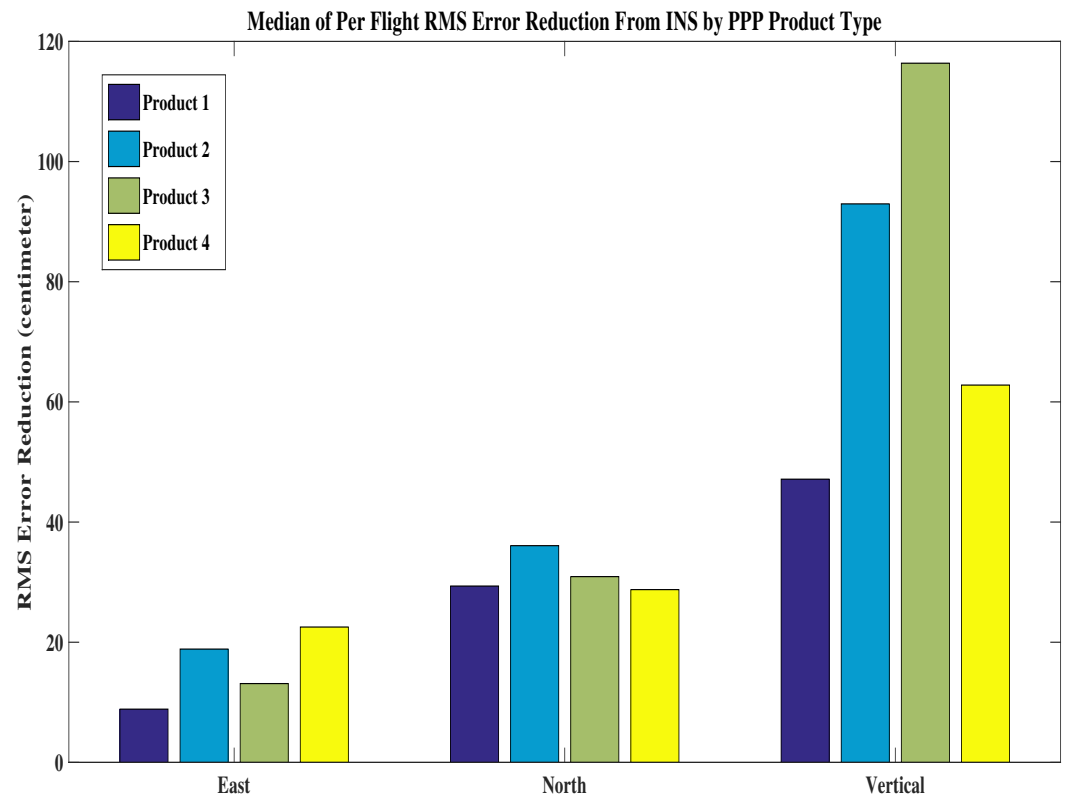

Figure 5.18: Median of Per Flight RMS Error Reduction from INS by PPP Product

trial. As shown in Fig. 5.19 roll, pitch and yaw are all estimated at sub-0.1 degree accuracy. A
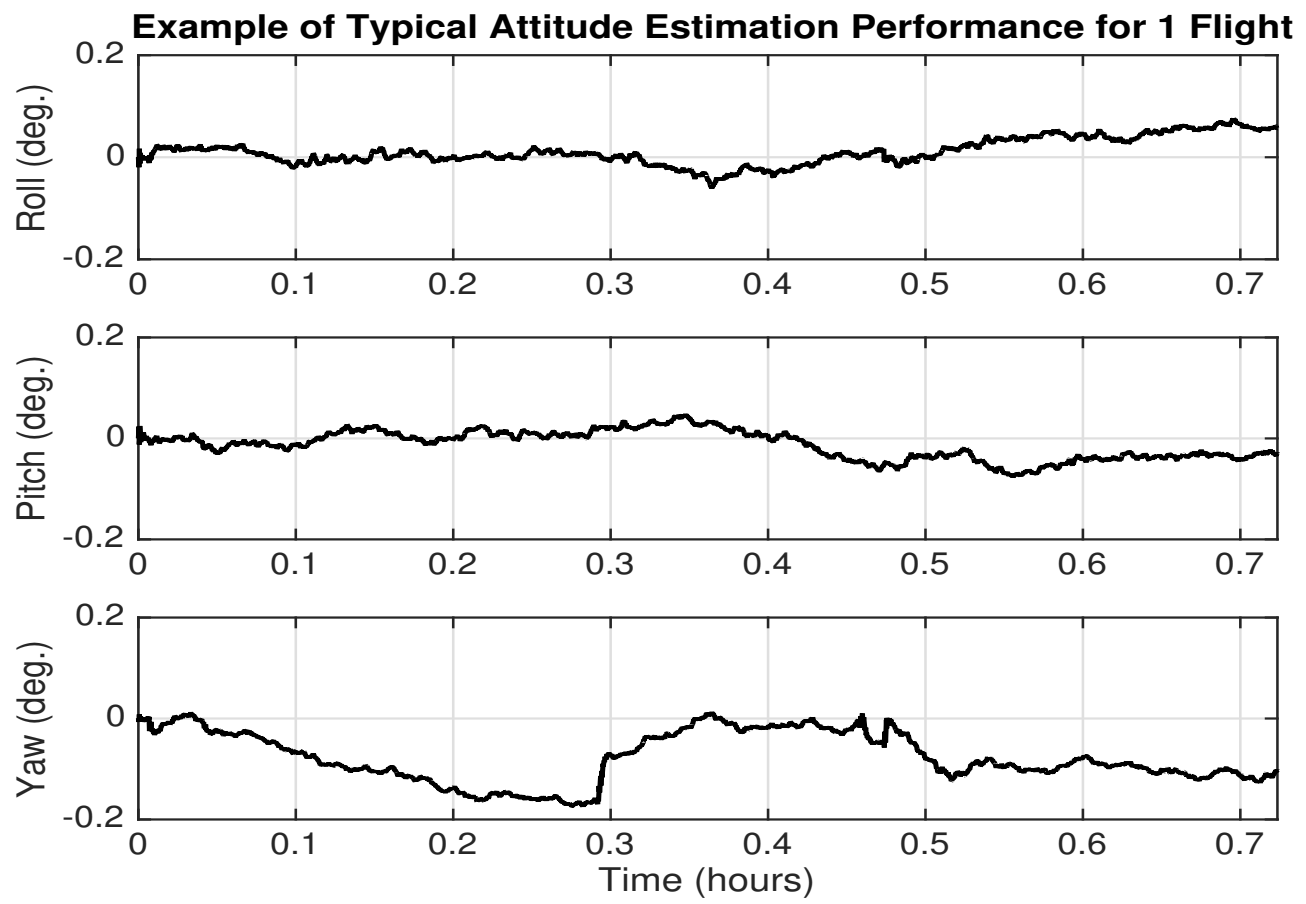

Figure 5.19: Example Attitude Estimation Error over a Single Flight

cumulative distribution of the attitude estimation performance of the 100 trials is shown in Fig. 5.20. As illustrated in Fig. 5.20 the median attitude estimation error is a few hundredths of a 


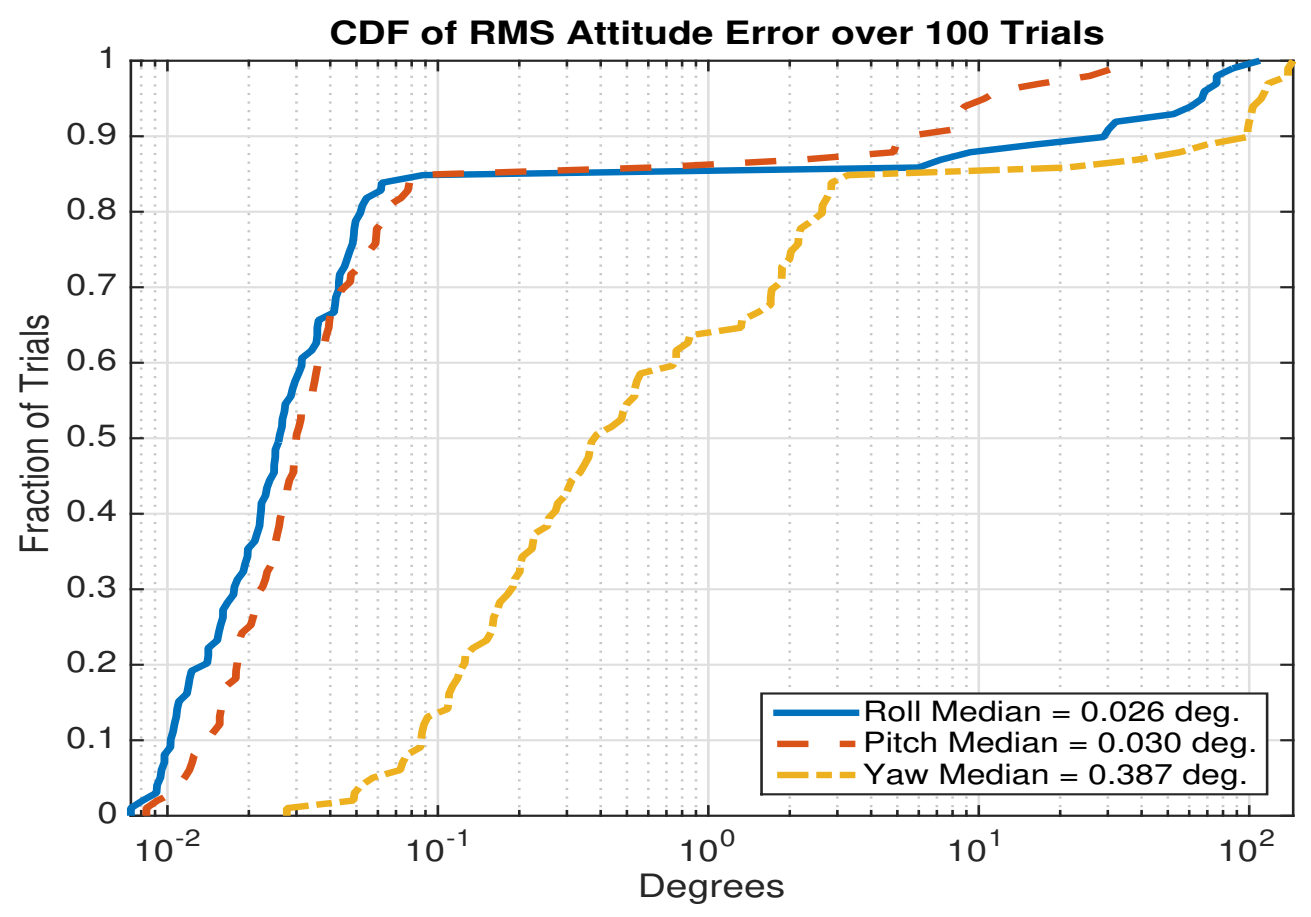

Figure 5.20: Cumulative Attitude Estimation Performance over 100 Flights

degree for roll and pitch, and an order of magnitude worse for the yaw angle. Poor yaw estimation is expected for flight profiles that are predominately steady-level flight. Just as in positioning, there are a handful of PPP/INS flights that have poor attitude estimation performance.

\subsubsection{Solution Convergence}

As mentioned in the literature review, PPP convergence is often cited as a downside to the PPP approach. Figure 5.21 was created by averaging the absolute phase bias estimate from the true simulated phases biases, epoch wise, for all 100 trials. As shown in Figures 5.21, the PPP/INS solution converges quicker than the PPP-only solution, which is important for reaching the performance similar to that of RTK.

\subsubsection{Smoothness of Positioning Solution}

In general, the PPP/INS improvement is attributed to the smoother positioning performance. This claim can be substantiated by conducting a frequency analysis of the positioning error time-series. For example, Fig. 5.22 shows a periodogram of the amplitude of the position error in the verticalaxis for a typical flight trial. The periodogram can be evaluated up to $5 \mathrm{~Hz}$ since the positioning residuals are available at $10 \mathrm{~Hz}$ (i.e. the GPS update rate). As shown in Fig. 5.22, at the low 


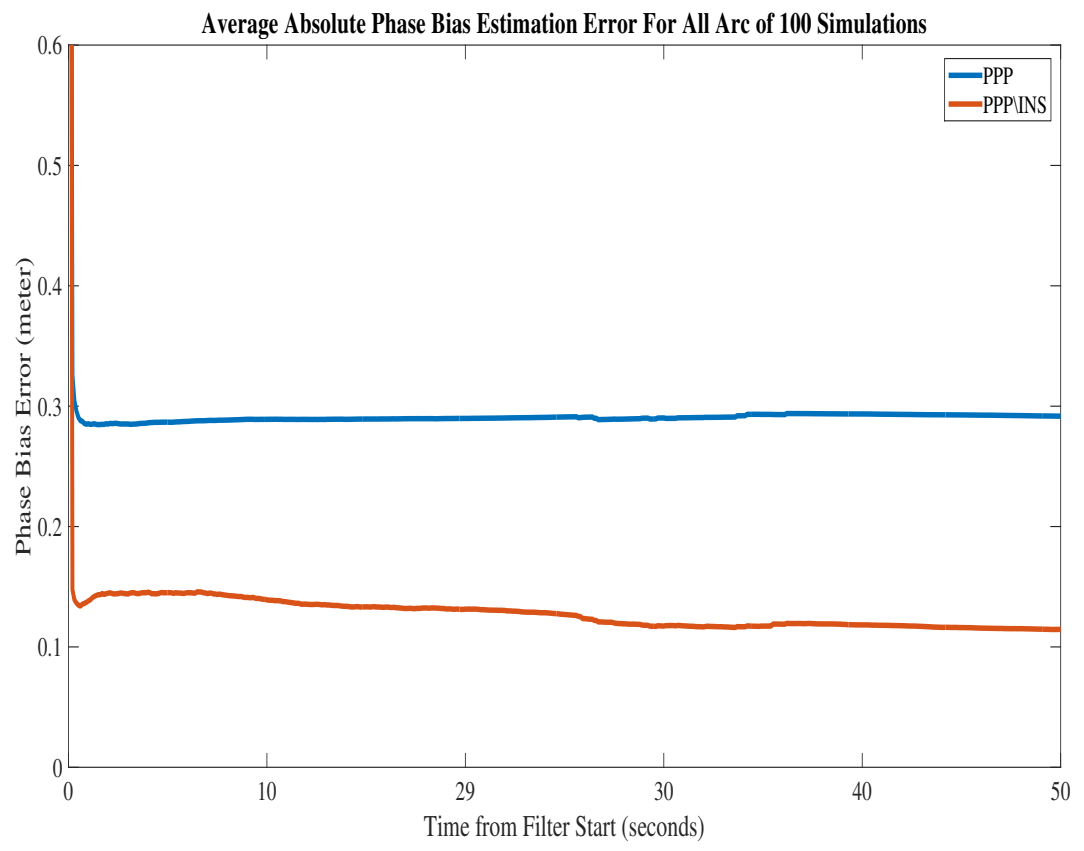

Figure 5.21: Average Absolute Phase Bias Estimation Error

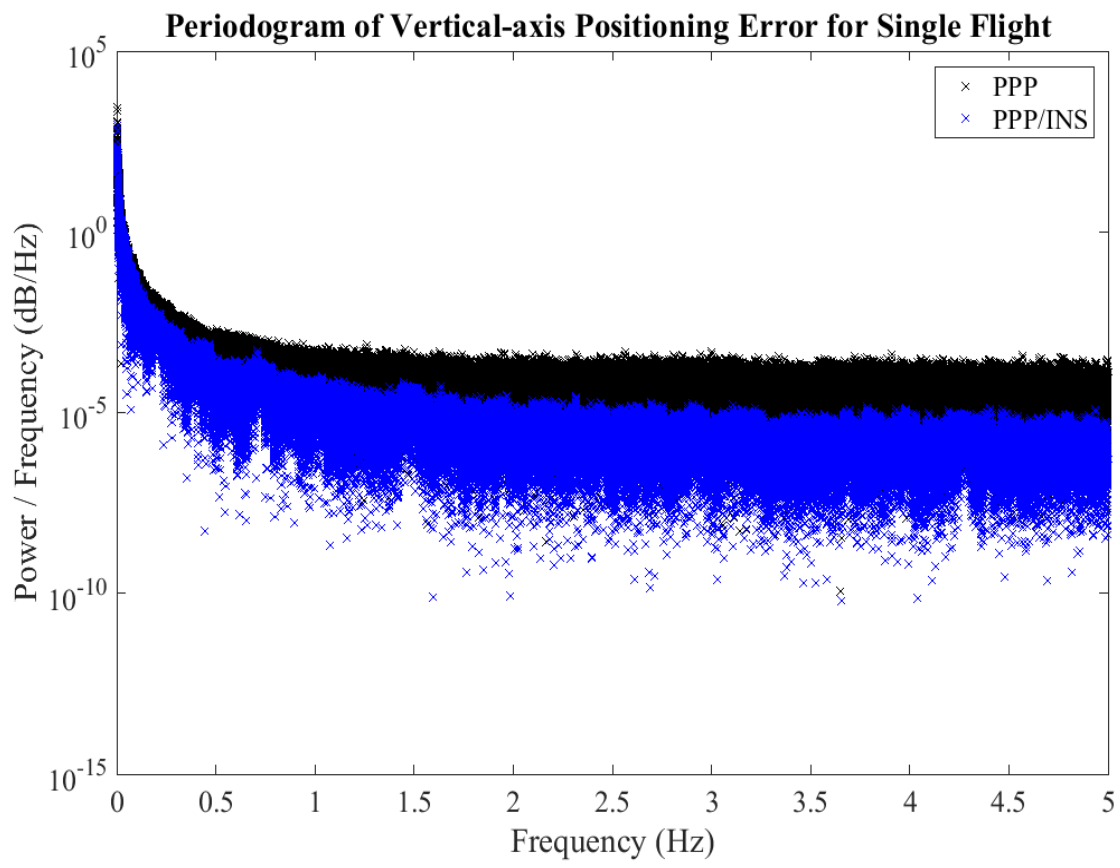

Figure 5.22: Periodogram of Vertical Position Error for Single Flight

frequencies the positioning performance is the same, and there is no noticeable difference in the absolute positioning performance, however, at the higher frequencies the PPP/INS error amplitude is reduced when compared to PPP. This analysis suggests smoother positioning from PPP/INS, 
which is critical for feedback control systems. 


\section{Chapter 6}

\section{Flight Data Results}

Parts of this chapter are reproduced from conference paper "Integration of Inertial Navigation into Real-Time GIPSY-x (RTGx)" [35], and journal paper "Flight Test Evaluation of Kinematic Precise Point Positioning of Small UAVs" [50]

\subsection{GIPSY-OASIS Overview}

GNSS-Inferred Positioning System and Orbit Analysis Simulation Software package (GIPSY-OASIS) has been in development at NASA JPL since the mine 1980's. GIPSY has been the primary geodetic and positioning software for NASA's TOPEX/Poseidon [51], JASON [52] and GRACE [53] low Earth orbiting spacecraft, and is operationally used to generate JPL's precise GPS orbits and clock products to the IGS [54]. GIPSY is licensed for free by Caltech to institutions for academic research purposes.

\subsection{RTGx Overview}

RTGx [11] is NASA JPL's revamped GNSS processing software, a rewrite of GIPSY in $C^{++}$, that can be configured for real-time or post-processed constellation orbit and clock determination, Low-Earth Orbiter (LEO) Precise Orbit Determination, or Precise Point Positioning (PPP). RTGx underlies the navigation software for the Air Force's next generation GPS operational control segment (OCX) [55]. In conjunction with JPL's GDGPS System, where RTGx generates the real-time GNSS orbit and clocks products, and is also the point-positioning engine, RTGx routinely and operationally produces sub-decimeter real-time kinematic positioning for a large number of GNSS tracking sites, 
globally [www.gdgps.net].

Different from its predecessor, RTGx now supports a multi-constellation GNSS processing capability, thus, an additional natural evolution of the RTGx software is to support an INS capability for kinematic applications that have demanding requirements. While tight GNSS/INS integration is by no means a new concept, integration of INS within RTGx inherits features that are already unique to JPL's RTGx processing strategy, such as single-receiver integer ambiguity resolution [14] as well as flexible and easily extendable parameter and model configuration. Incorporation of INS in RTGx will further enable research in more advanced INS/GNSS models, such as solving for deviations of the local gravity error, processing platforms with multiple antennas, leveraging atomic clocks, ingesting pressure sensor data for troposphere modeling, solving for unknown IMU lever arms, and processing kinematic platforms as part of a network solution, all of which which can contribute to increased accuracy required by various science applications.

\subsection{Data Sets}

Eight data sets were provided by a NGS,NOAA program called Gravity for the Redefinition of the American Vertical Datum (GRAV-D). These are multiple hour long flight collected data sets, where each data set contains

- $200 \mathrm{~Hz}$ raw IMU measurements,

- $1 \mathrm{~Hz}$ raw GPS observables (pseudorange and carrier-phase),

- lever arm between the platform's instrument and GPS Antenna, and between the instrument and the IMU

- and the commercial GPS/INS smoothed attitude estimate.

The location, date, and duration of each of the flights is summarized in Table 6.1.

Table 6.1: Flight Collected Data Set Durations

\begin{tabular}{|c|l|l|}
\hline Location & Date & Duration \\
\hline \hline Alaska & $29-$ May-2015 & $3 \mathrm{hr} 54 \mathrm{~min}$ \\
\hline Alaska & $9-J u n e-2015$ & $4 \mathrm{hr} 46 \mathrm{~min}$ \\
\hline Louisiana & 23 -Oct-2008 & $2 \mathrm{hr} 27 \mathrm{~min}$ \\
\hline Louisiana & $19-$ Nov-2008 & $3 \mathrm{hr} 19 \mathrm{~min}$ \\
\hline New York & $10-$ Aug-2011 & $4 \mathrm{hr} 4 \mathrm{~min}$ \\
\hline New York & $18-$ Aug-2011 & $3 \mathrm{hr} 43 \mathrm{~min}$ \\
\hline
\end{tabular}


These eight data sets come from six flights. Where two flights contain an Applanix system, two flights contain a Novatel system, and the final two flights fly both the Applanix and Novatel systems. These last two data sets are valuable because they allow for the direct comparison of two IMUs for the same flight. Table 6.2 summarizes the GPS/INS system and lever arm used for each flight.

Table 6.2: GPS/INS System for Flight Collected Data

\begin{tabular}{|c|l|l|l|}
\hline Location & GPS/INS System & Lever Arm $(\mathbf{m})$ \\
\hline \hline Alaska & $\begin{array}{l}\text { Novatel SPAN (navigation } \\
\text { grade) }\end{array}$ & $\mathrm{x}=-0.132, \mathrm{y}=0.095, \mathrm{z}=0.463$ \\
\hline Alaska & $\begin{array}{l}\text { Novatel SPAN (navigation } \\
\text { grade) }\end{array}$ & $\mathrm{x}=-0.132, \mathrm{y}=0.095, \mathrm{z}=0.463$ \\
\hline Louisiana & $\begin{array}{l}\text { Applanix POS-AV (tatical } \\
\text { grade) }\end{array}$ & $\mathrm{x}=2.72, \mathrm{y}=-0.05, \mathrm{z}=-0.5$ \\
\hline Louisiana & $\begin{array}{l}\text { Applanix POS-AV (tatical } \\
\text { grade) }\end{array}$ & $\mathrm{x}=2.72, \mathrm{y}=-0.05, \mathrm{z}=-0.5$ \\
\hline New York & Novatel and Applanix System & $\begin{array}{l}\text { Novatel }-\mathrm{x}=-1.5, \mathrm{y}=0.02, \mathrm{z}=0.56 \\
\text { Applanix }-\mathrm{x}=-1.7, \mathrm{y}=0.2, \mathrm{z}=-0.56\end{array}$ \\
\hline New York & Novatel and Applanix System & $\begin{array}{l}\text { Novatel }-\mathrm{x}=-1.5, \mathrm{y}=0.02, \mathrm{z}=0.56 \\
\text { Applanix }-\mathrm{x}=-1.7, \mathrm{y}=0.2, \mathrm{z}=-0.56\end{array}$ \\
\hline
\end{tabular}

An example of the flight data can be seen below in Fig. 6.1 and Fig. 6.2. The rapid accent and decent in Fig.6.1 makes the data sets particularly challenging for handling the tropospheric delay.

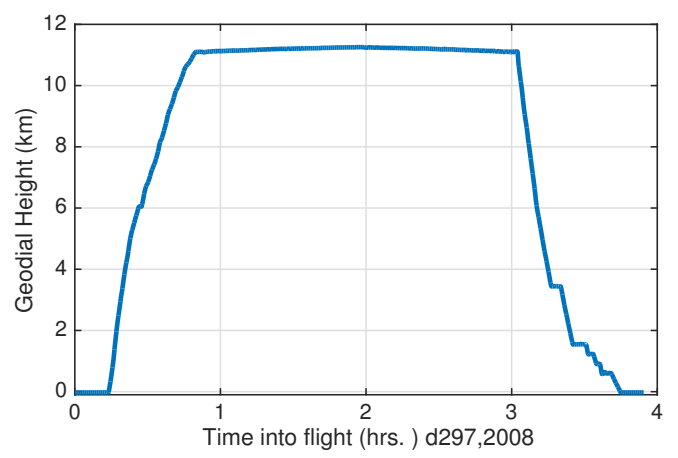

Figure 6.1: Altitude profile of the d297,y2008 GRAV-D data-set. 


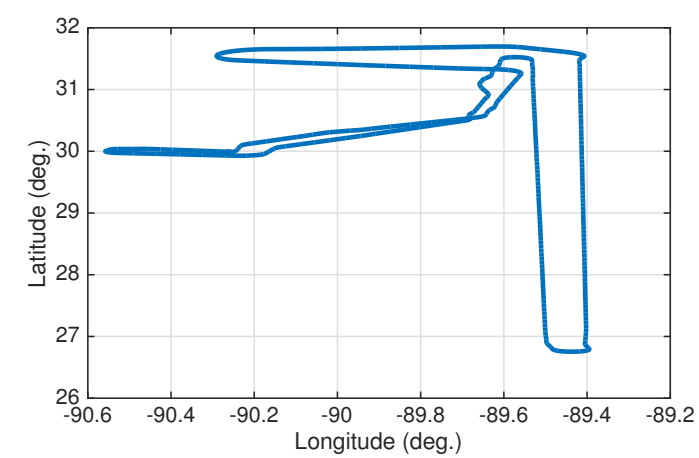

Figure 6.2: Lat/Lon profile of d297,y2008 GRAV-D data-set.

\subsection{Processing Strageties}

\subsubsection{GIPSY-OASIS Reference Solution Processing Stragety}

The reference solution for this analysis uses Caltech JPL's GIPSY-OASIS 6.2 software [56], which is licensed for free by Caltech for academic research purposes. This software package has been used extensively for geodetic and positioning applications on NASA missions: TOPEX/Poseidon [51], JASON [52] and GRACE [53] low Earth orbiting spacecraft. GIPSY is also used to generate JPL's precise GPS orbits and clock products to the IGS [54]. GIPSY uses a square root information filter [57] to estimate parameters of interest.

Because the data sets are kinematic, the reference processing strategy is to iterate over the position solution while varying the GIPSY configuration parameters to eliminate data outliers. A block diagram of the processing strategy is shown in Figure 6.3, which requires defining some GIPSY terms:

- GNSS Data to Positioning (GD2P): GIPSY's main user interface script for PPP.

- PseudoRange Data to Positioning (PR2P): GIPSY's script for pseudorange only point positioning.

- Time Dependent Parameter (TDP): GIPSY's output format for positioning solutions and other solved for parameters (e.g. clock biases, troposphere, phase biases).

- QM File GIPSY's native binary GNSS measurement format.

As can be seen in Fig. 6.3, before the first iteration, the Receiver Independent Exchange Format (RINEX) file is converted to a GIPY binary QM file. During this process, a GNSS data editor is used to flag carrier-phase breaks and remove gross data outliers [58]. With the generated QM 


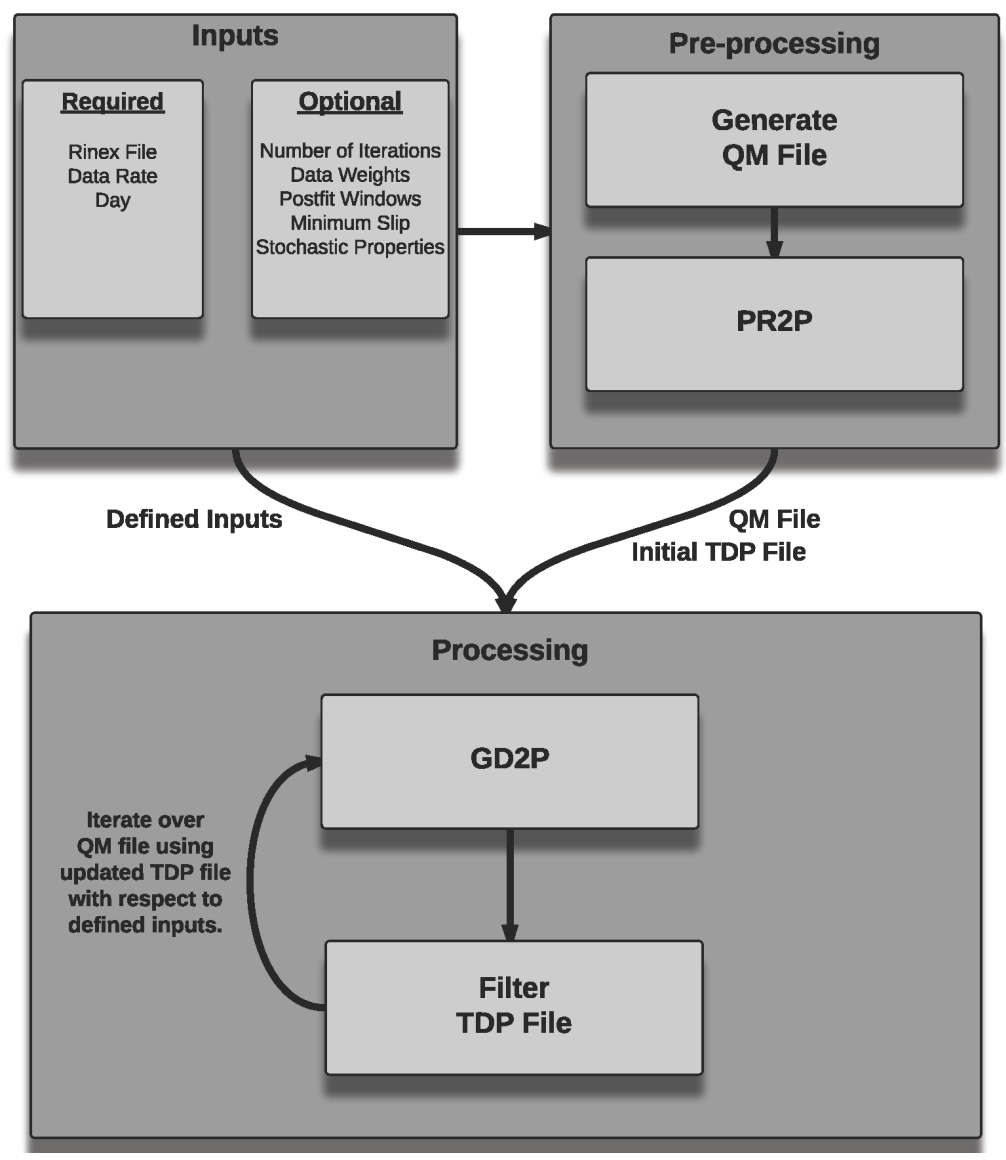

Figure 6.3: Kinematic PPP Strategy with GIPSY OASIS. A wrapper software is used to interactively process the position solution.

file, the first iteration position estimation is generated using only the pseudorange observable with GIPSY's PR2P. For the remaining iterations, a subset of GIPSY processing options are varied while iterating over the previous position solution (TDP file) as the a priori position solution. Within Figure 6.3, the configuration options that are varied for each run are as follows:

- Data Weights: Relative scaling of the measurement noise between the pseudorange and carrier-phase measurements. For this application, the weighting started at one-to-one and ended at one-hundred-to-one.

- Postfit Residual Window: Within each GIPSY processing run, multiple passes of a Kalman filter and smoother are conducted. Between each pass, postfit data residuals are evaluated and data are marked outlier based on defined thresholds and excluded from the next pass. At each pass, the residuals of all data, inlier and outlier, are evaluated and either added back in order excluded from the run. This process is repeated until all data meet the postfit window criteria or a maximum number of iterations are exceeded. 
- Stochastic Models: The position and wet troposphere delay estimates can be modeled using either white noise about the nominal solution or random walk process noise. Additionally, the a priori $\sigma$ and magnitude and rate of process noise updates can be set.

- Minimum Slip: After each filter iteration, jumps in the post-fit phase residuals are used to identify the possibility of a carrier-phase break that was missed by the data editor. New breaks are flagged for the next iteration

In addition to the configuration parameters listed above that are varied for each iteration, several other GIPSY options were selected and held fixed in this study. In particular, the VMF1 [27] model was used as the troposphere mapping function and nominal estimation. For the several remaining available GD2P options (e.g. elevation cutoff, tide models, etc.), the defaults provided by JPL were used.

\subsubsection{RTGx Base Processing Stragety}

Within this section, the RTGx processing stragety adopted is described. In this analysis, two solutions are compared to the reference position solutions, namely K-PPP with INS and K-PPP without INS. The analysis considers the performance of the forward-filter only solutions, as this is most applicable to the real-time needs of the target application.

For both filter-only K-PPP strategies, prior to processing with RTGx, the RINEX data were pre-processed by GIPSY's GPS data editor in order to remove gross-outliers and flag carrier-phase breaks [58]. In addition a simple troposphere estimation strategy that solves for a residual wet zenith delay as a random walk parameter was adopted. This value was empirically tuned to provide smooth estimation performance when at altitude and selected to be $5 \mathrm{e}-5 \frac{m}{\sqrt{s}}$. Furthermore, for both cases, with and without INS, the GPS receiver clock was modeled as a random walk process with $1000.0 \frac{m}{\sqrt{s}}$. Finally, all solutions used JPL's final orbit/clock submissions to the International GNSS Service [54].

For designing the INS stochastic models, typically the sensor specs of the individual IMU sensors (e.g. Angular Random Walk, Velocity Random Walk, bias-instabilities) would be used to drive these models [59]. However, for the Applanix PosAV GPS/IMU system used in the Kinematic Challenge data set, these IMU sensor parameters were unknown. Therefore, these parameters were initially selected assuming an intermediate grade IMU and then empirically tuned.

Finally, in order to provide better comparison and aid in the convergence of the GPS-only K-PPP strategy, a dual-frequency pseudorange-only solution was ingested as the a priori nominal position 
solution with the filter-only runs. The carrier-phase based K-PPP positions were then solved as for assuming 5 meters of uncertainty in the nominal solution at each data epoch.

\subsection{Results}

The key statistics with respect to positioning accuracy and attitude estimation are summarized in this section. For positioning, the metrics selected include:

- Root Sum Square (RSS)

- Root Mean Square (RMS)

- $\operatorname{Median}(\widetilde{x})$

- Standard Deviation $(\sigma)$

- Average Bias (b)

- Max absolute error $(\operatorname{Max}(|b|))$

For this analysis, all errors are reported with respect to the reference solutions. Additionally, these metrics are reported for the data sets both before and after the initial solution convergence until the end of the data sets. The convergence period was excluded because the most crucial period during airborne science campaigns is after ascending to the final altitude. In this analysis, this was done by simply by evaluating errors over only the last $1 / 2$ of each flight.

\subsubsection{Base Run}

The CDF of the positioning error for the eight data sets using the base run parameter configuration can be seen in Fig. 6.4. This plot shows both the full statistics, and the statistics after the carrierphase biases have converged (i.e. the last half of the flight). From this plot it should be noted that the PPP only filter outperforms the PPP/INS filter with respect to the full statistics; however, after the solution convergence the PPP/INS filter provides a smaller positioning error.

In addition to the $\mathrm{CDF}$ for the base run parameter configuration, the full statistics for the eight data sets can be seen in Table 6.3. From this table it should be noted that PPP/INS filter provides a substantially smaller maximum error. This is an important characteristic for feed-back systems as larger positioning errors cause erronous acceleration estimates. Additionally, the statistics for the base run parameter after the convergience of the carrier-phase biases can be seen in table 6.4. 

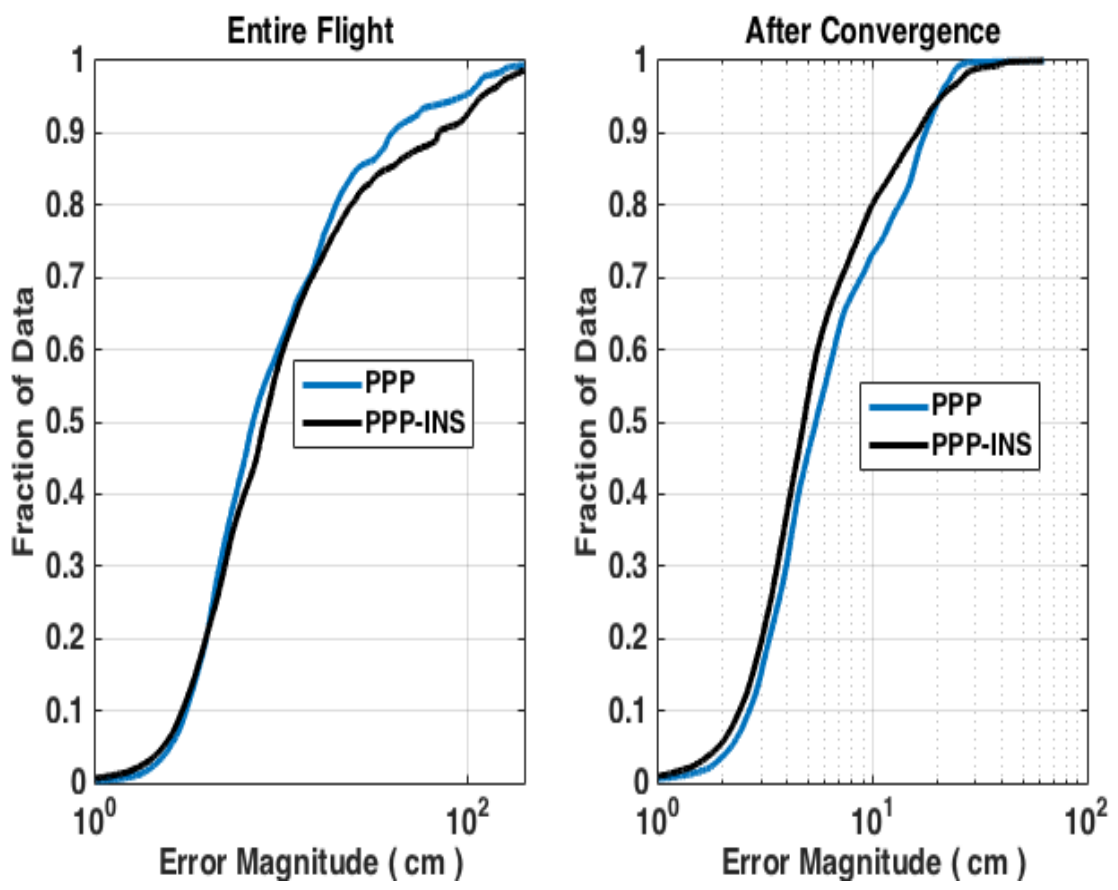

Figure 6.4: CDF of RSS Positioning Error for Base Configuration

Table 6.3: Statistics of positioning performance for full baserun configuration (flight data)

\begin{tabular}{|c|c|c|}
\hline Filter-Only & K-PPP & K-PPP/INS \\
\hline Median $\mathbf{( c m})$ & 12.10 & 14.67 \\
\hline S.D $\sigma(\mathbf{c m})$ & 63.94 & 77.65 \\
\hline Bias $b(\mathbf{c m})$ & 32.33 & 43.23 \\
\hline Max $(|b|)(\mathbf{c m})$ & 1105.21 & 507.18 \\
\hline
\end{tabular}

Table 6.4: Statistics of positioning performance after convergence for baserun configuration (flight data)

\begin{tabular}{|c|c|c|}
\hline Filter-Only & K-PPP & K-PPP/INS \\
\hline Median $\mathbf{( c m})$ & 9.37 & 8.34 \\
\hline S.D $\sigma(\mathbf{c m})$ & 10.58 & 11.29 \\
\hline Bias $b(\mathbf{c m})$ & 13.67 & 12.40 \\
\hline Max $(|b|)(\mathbf{c m})$ & 109.32 & 108.28 \\
\hline
\end{tabular}

\subsubsection{Linearize About Previous State Estimate}

As noted above, the PPP only solution is provided with an input time series of position estimates. This time series was generated with PR2P which used only the pseudorange measurements.

For this comparision, the PPP only filter is not provided an input time series of position estimates. Instead, both the PPP and the PPP/INS filters use their previous state estimate as their linearization nominal. This sections consideres two different configurations: using pseudorange and carrier-phase data while both filters linearize about the previous state estimate, and using only pseudoragne while 
both filters linearize about the previous stae estimate.

In Fig. 6.5, the CDF of the RSS positioning error for the eight data sets when both filters using both carrier-phase and psuedorange observables while using the previous state estimate as a linearization nominal can be seen. From Fig. 6.5 it should be noted that the PPP/INS filter outperforms the PPP only filter with respect to positioning error both before and after the solution convergence. The statistics for both the PPP only and the PPP/INS filters can be seen in Table 6.5 and Table 6.6 for the full flight statistics and the statistics after convergence, respectively.
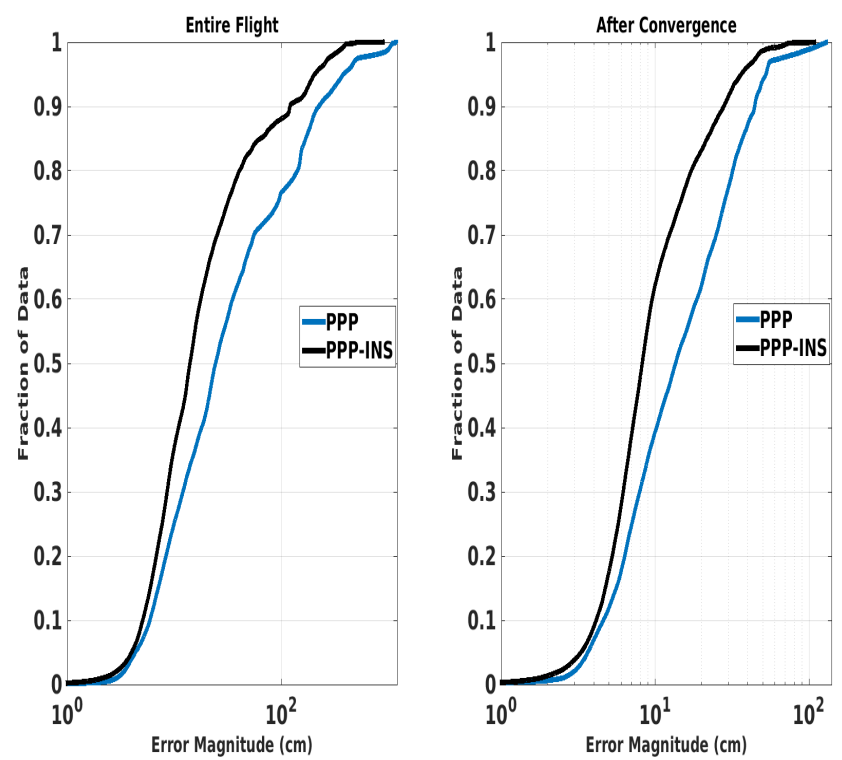

Figure 6.5: CDF of RSS positioning error without input position time-series using PC-LC observables

Table 6.5: Statistics of positioning performance for full PC-LC previous epoch linearization configuration (flight data)

\begin{tabular}{|c|c|c|}
\hline Filter-Only & K-PPP & K-PPP/INS \\
\hline Median $(\mathbf{c m})$ & 24.15 & 14.67 \\
\hline S.D $\sigma(\mathbf{c m})$ & 165.45 & 77.65 \\
\hline Bias $b(\mathbf{c m})$ & 88.26 & 43.23 \\
\hline Max $(|b|)(\mathbf{c m})$ & 1188.42 & 507.18 \\
\hline
\end{tabular}

Table 6.6: Statistics of positioning performance after convergence for previous epoch linearization configuration (flight data)

\begin{tabular}{|c|c|c|}
\hline Filter-Only & K-PPP & K-PPP/INS \\
\hline Median $(\mathbf{c m})$ & 13.56 & 8.34 \\
\hline S.D $\sigma(\mathbf{c m})$ & 18.72 & 11.29 \\
\hline Bias $b(\mathbf{c m})$ & 20.17 & 12.40 \\
\hline Max $(|b|)(\mathbf{c m})$ & 129.26 & 108.28 \\
\hline
\end{tabular}


The next figure, Fig. 6.6, shows the CDF of the RSS positioning error for both the PPP only and the PPP/INS when only using the pseudorange obserable and the previous state estimate as the linearization nominal. With this configuration it should be noted that there is a substancial benefit to PPP/INS filter. This can be seen by the large median error positioning error reduction both before and after solution convergence, as shown in Table 6.7 and Table 6.8, respectively. In addition to the median error, it should be noted the standard deviation, bias, and maximum error are all substancially smaller for the PPP/INS filter as shown in Table 6.7 and Table 6.8.

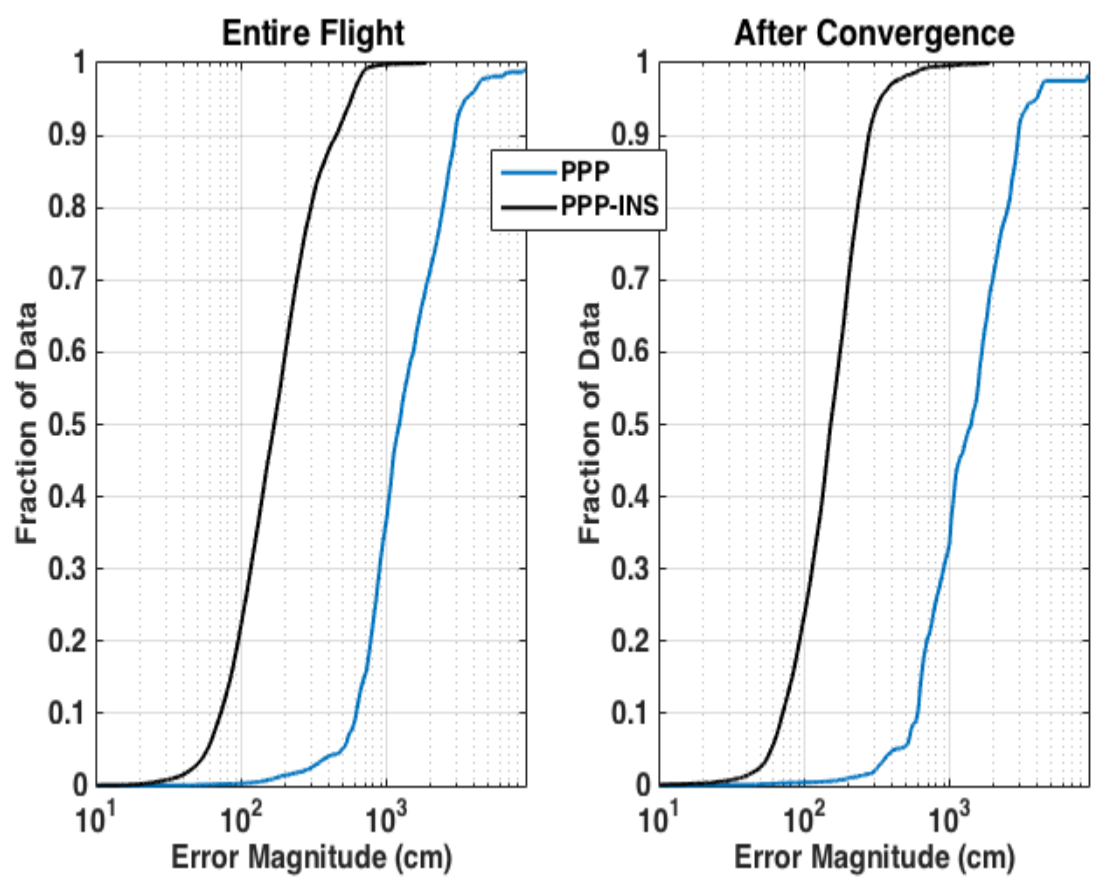

Figure 6.6: CDF of RSS positioning error without input position time-series using only PC observables

Table 6.7: Statistics of positioning performance for full PC-only previous epoch linearization configuration (flight data)

\begin{tabular}{|c|c|c|}
\hline Filter-Only & K-PPP & K-PPP/INS \\
\hline Median (m) & 20.98 & 2.88 \\
\hline S.D $\sigma(\mathbf{m})$ & 47.77 & 2.75 \\
\hline Bias $b(\mathbf{m})$ & 30.22 & 3.68 \\
\hline Max $(|b|)(\mathbf{m})$ & 84.08 & 32.43 \\
\hline
\end{tabular}


Table 6.8: Statistics of positioning performance for last-half of previous epoch linearization configuration (flight data)

\begin{tabular}{|c|c|c|}
\hline Filter-Only & K-PPP & K-PPP/INS \\
\hline Median $\mathbf{( c m})$ & 18.68 & 2.70 \\
\hline S.D $\sigma(\mathbf{c m})$ & 47.31 & 2.56 \\
\hline Bias $b(\mathbf{c m})$ & 28.35 & 3.43 \\
\hline Max $(|b|)(\mathbf{c m})$ & 84.08 & 32.43 \\
\hline
\end{tabular}

\subsubsection{Troposphere Model}

As stated above, the troposphere is a hard to mitigate GPS error source with several models and mapping functions. Because of this, it is useful to see the effect that different troposphere models have on the ability to estimate position. For this anaylsis, four troposphere models are used: no nominal with a Niell mapping function, static height nominal with a Niell mapping function, GPT2 nominal with a GMF mapping function, and a VMF1 nominal with a VMF1 mapping.

The maximum RSS positioning error with respect to the tropopshere model used can be seen in Fig. 6.7. From this figure it can be seen that the PPP/INS filter has a smaller maximum RSS positioning error for the all of the troposphere models used with the exception being the runs where no nominal troposphere is provided. This can also be seen in Table 6.9 which provides the full run statistics with respect to the troposphere model used.

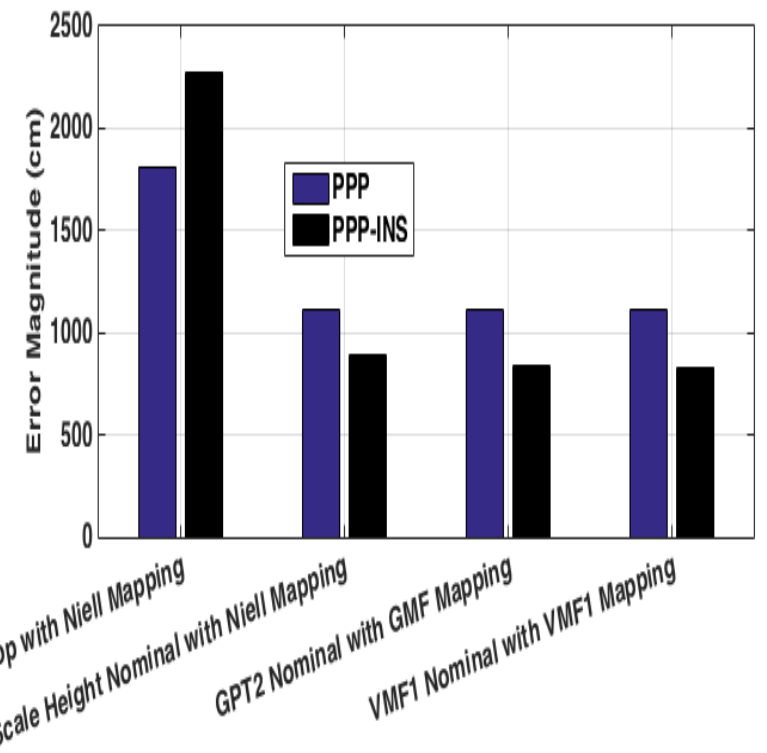

Figure 6.7: Max positioning as a function of troposphere model

The median of the RSS positioning error with respect to the troposphere model used can be seen in Fig. 6.8. With respect to the median, the PPP/INS filter only out performs the PPP filter in the 
case where no nominal troposphere is provided. This implies the high fedility the GPS models are, the less INS is needed for position estimation.

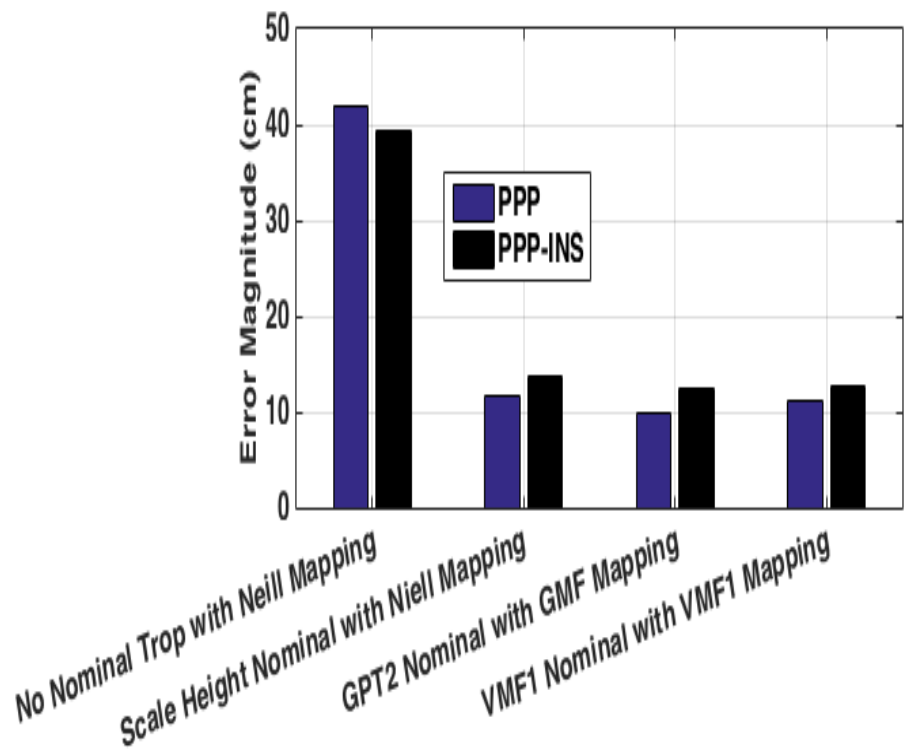

Figure 6.8: Median of positioning error as a function of troposphere model

Table 6.9: Statistics of positioning performance for different troposphere models (flight data)

\begin{tabular}{|c|c|c|}
\hline Filter-Only & K-PPP & K-PPP/INS \\
\hline Median No Nominal $(\mathrm{cm})$ & 42.61 & 39.48 \\
\hline Median Static Height (cm) & 11.77 & 13.74 \\
\hline Median GPT2 (cm) & 9.90 & 12.51 \\
\hline Median VMF1 (cm) & 11.21 & 12.67 \\
\hline S.D No Nominal $\sigma(\mathbf{c m})$ & 41.12 & 36.33 \\
\hline S.D Static Height $\sigma(\mathbf{c m})$ & 10.12 & 11.34 \\
\hline S.D GPT2 $\sigma(\mathbf{c m})$ & 8.38 & 10.15 \\
\hline S.D VMF1 $\sigma(\mathrm{cm})$ & 10.21 & 10.59 \\
\hline Bias No Nominal $b(\mathbf{c m})$ & 44.12 & 42.76 \\
\hline Bias Static Height $b(\mathrm{~cm})$ & 32.33 & 43.23 \\
\hline Bias GPT2 $b(\mathrm{~cm})$ & 31.23 & 42.15 \\
\hline Bias VMF1 $b(\mathbf{c m})$ & 31.07 & 44.34 \\
\hline Max No Nominal $(|b|)(\mathbf{c m})$ & 1814.21 & 2277.25 \\
\hline Max Static Height $(|b|)(\mathbf{c m})$ & 1100.23 & 886.46 \\
\hline Max GPT2 $(|b|)(\mathbf{c m})$ & 1111.12 & 839.61 \\
\hline Max VMF1 $(|b|)(\mathbf{c m})$ & 1091.14 & 836.67 \\
\hline
\end{tabular}

\subsubsection{Positioning Smoothness}

As stated above, the PPP/INS improvement is attributed to the smoother positioning performance as the INS is essential interpolating between GPS updates. Again, this claim is substantiated 
by conducting a frequency analysis of the RSS positioning error time-series. Figure 6.9 shows a periodogram of the amplitude of the RSS position error with respect to the nominal value that is predicted with the RTGx models for all eight flights. This periodogram can be evaluated up to $0.5 \mathrm{~Hz}$ since the GPS observables were collected at at $1 \mathrm{~Hz}$ interval.

As shown in Fig. 6.9, at both the low and high frequencies the PPP/INS error amplitude is reduced when compared to PPP. This analysis suggests smoother positioning from PPP/INS, which is critical for feedback control systems. Additionally, by looking at the error with respect to the nominal, it can be seen that the PPP/INS filter more closely matches what the high fidelity models are predicting.

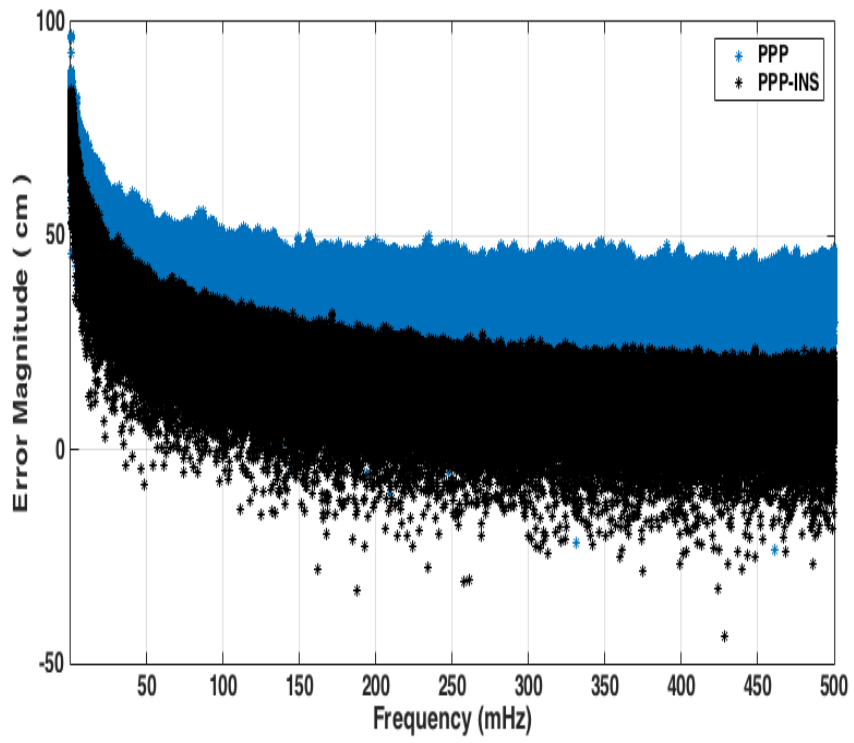

Figure 6.9: Periodogram of Vertical Position Error for Single Flight

\subsubsection{Attitude Estimation}

As another method to validate the implementation of INS into RTGx the estimated attitude is compared to the smoothed commercial solution. This attitude comparision for all data sets can be see in 6.10. Additionally, Table 6.10 shows very good agreement with respect to the attitude provided by the commercial solution. It should be noted that for flights that are primarily flying straight and level that it is well known that the IMU yaw-bias is most difficult to observe. 


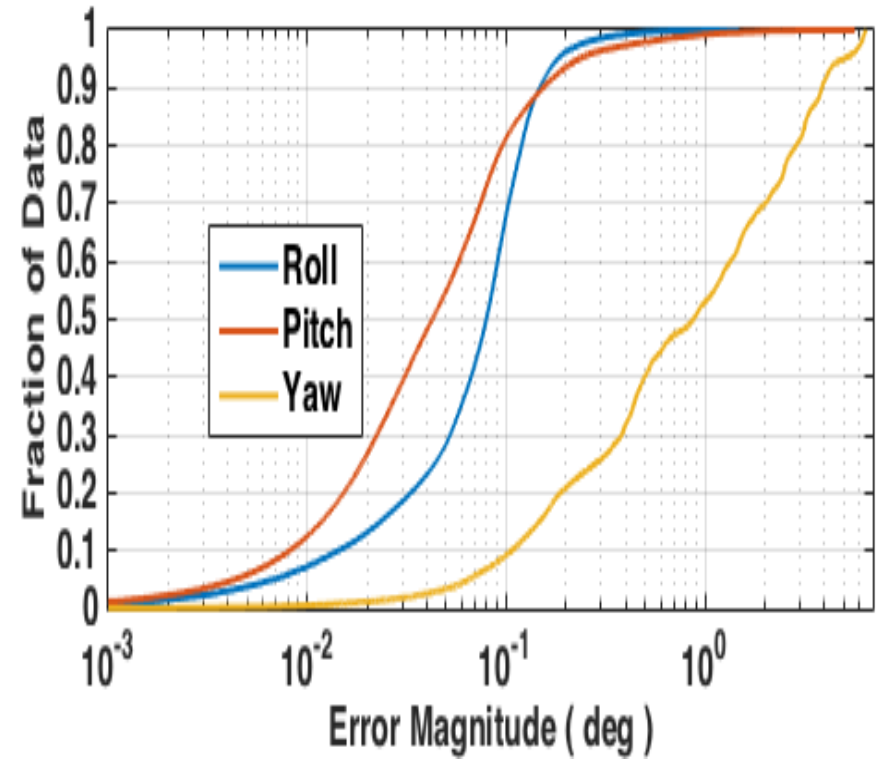

Figure 6.10: CDF of total attitude statistics. Includes data from the full duration of all eight data sets.

Table 6.10: Statistics of attitude performance for all flight data with baserun configuration (flight data)

\begin{tabular}{|c|c|}
\hline Filter-Only & K-PPP /INS \\
\hline Median Roll (deg) & 0.014 \\
\hline Median Pitch (deg) & 0.010 \\
\hline Median Yaw(deg) & 0.012 \\
\hline S.D Roll $\sigma$ (deg) & 0.110 \\
\hline S.D Pitch $\sigma$ (deg) & 0.198 \\
\hline S.D Yaw $\sigma$ (deg) & 2.196 \\
\hline Bias Roll $b$ (deg) & 0.004 \\
\hline Bias Pitch $b$ (deg) & 0.002 \\
\hline Bias Yaw $b$ (deg) & 0.182 \\
\hline Max Roll $(|b|)(\mathbf{d e g})$ & 1.352 \\
\hline Max Pitch $(|b|)$ (deg) & 4.655 \\
\hline Max Yaw $(|b|)$ (deg) & 4.522 \\
\hline
\end{tabular}

As stated above, for the eight data sets, two different INS were flown. The first systems is the Novatel SPAN package with a navigation grade IMU. The second system is the Applanix Pos-AV package with a tatical grade IMU. Now, we will look at the attitude estimate with respect to the grade of the IMU. The CDF of the RMS attitude error for the navigation grade and tatical grade IMUs can be seen in Fig. 6.11 and Fig. 6.12, respectively. The total statsitcs can be seen in Table 6.11 and Table 6.12 for the two systems. From Table 6.11 and Table 6.12 it should be noted that while both shows very good agreement with the smoothed attitude solution, the tatical grade IMU performs better, specifically with respect to yaw estimation. 


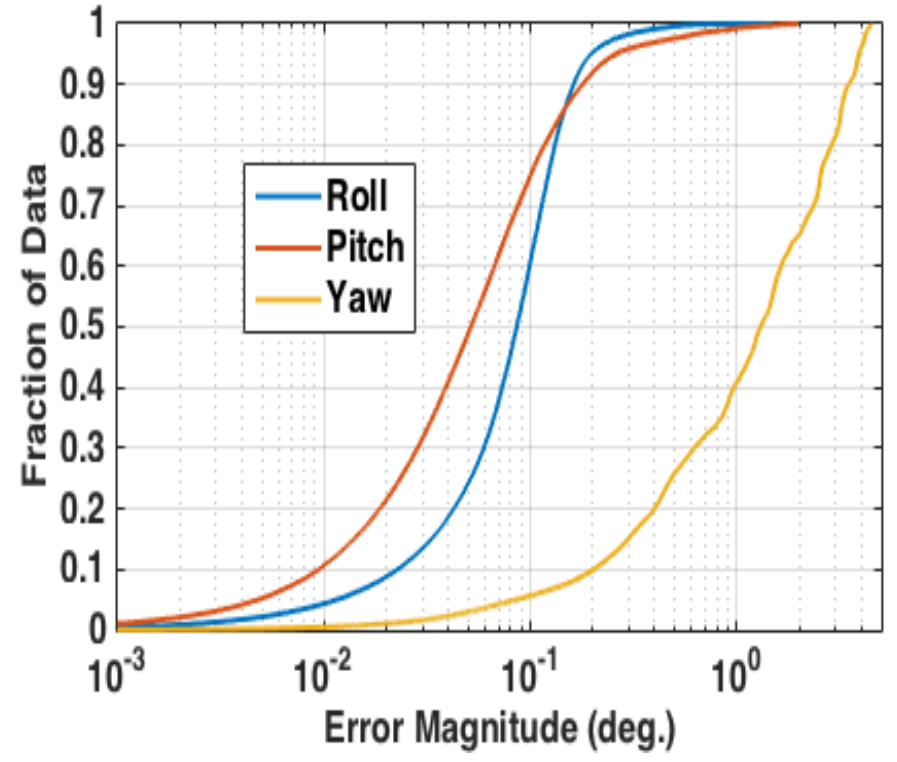

Figure 6.11: Navigation Grade IMU Attitude Statistics for Base Run Configuration

Table 6.11: Statistics of attitude performance for navigation grade IMU for all fligth data with baserun configuration (flight data)

\begin{tabular}{|c|c|}
\hline Filter-Only & K-PPP/INS \\
\hline Median Roll (deg) & 0.019 \\
\hline Median Pitch (deg) & 0.019 \\
\hline Median Yaw (deg) & 1.180 \\
\hline S.D Roll $\sigma$ (deg) & 0.118 \\
\hline S.D Pitch $\sigma$ (deg) & 0.181 \\
\hline S.D Yaw $\sigma$ (deg) & 1.544 \\
\hline Bias Roll $b(\mathbf{d e g})$ & 0.009 \\
\hline Bias Pitch $b(\mathbf{d e g})$ & 0.016 \\
\hline Bias Yaw $b(\mathbf{d e g})$ & 1.300 \\
\hline Max Roll $(|b|)(\mathbf{d e g})$ & 0.895 \\
\hline Max Pitch $(|b|)(\mathbf{d e g})$ & 1.986 \\
\hline Max Yaw $(|b|)(\mathbf{d e g})$ & 4.522 \\
\hline
\end{tabular}




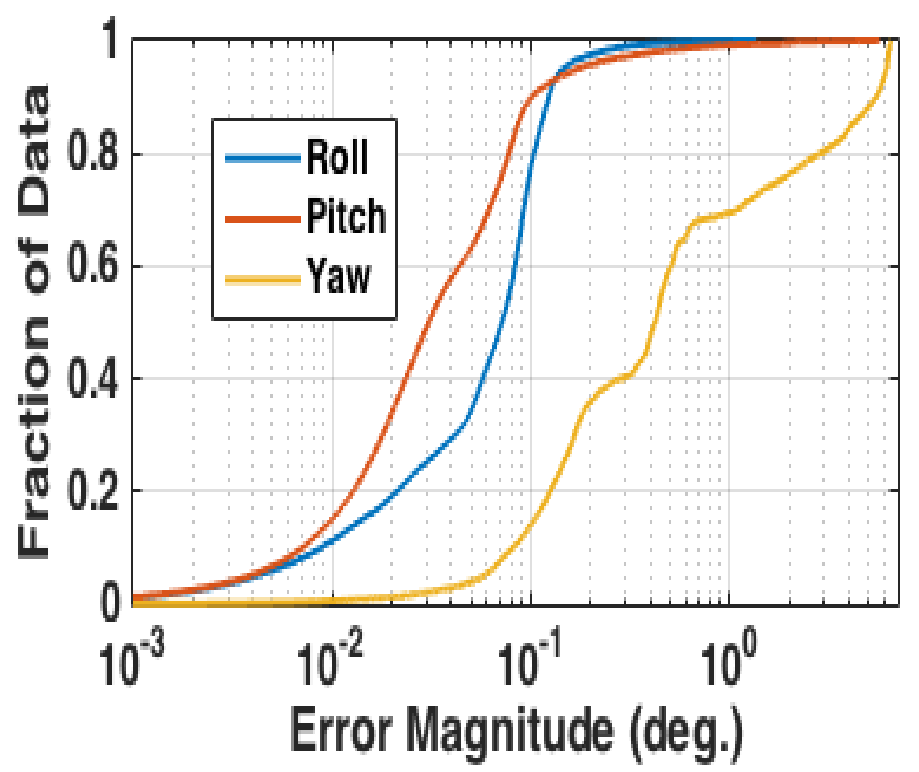

Figure 6.12: Tatical Grade IMU Attitude Statistics for Base Run Configuration

Table 6.12: Statistics of attitude performance for tatical grade IMU for all flight data with base run configuration (flight data)

\begin{tabular}{|c|c|}
\hline Filter-Only & K-PPP/INS \\
\hline Median Roll (deg) & 0.012 \\
\hline Median Pitch (deg) & 0.024 \\
\hline Median Yaw(deg) & 0.395 \\
\hline S.D Roll $\sigma(\mathbf{d e g})$ & 0.096 \\
\hline S.D Pitch $\sigma(\mathbf{d e g})$ & 0.216 \\
\hline S.D Yaw $\sigma(\mathbf{d e g})$ & 2.027 \\
\hline Bias Roll $b(\mathbf{d e g})$ & 0.002 \\
\hline Bias Pitch $b(\mathbf{d e g})$ & 0.029 \\
\hline Bias Yaw $b(\mathbf{d e g})$ & 1.348 \\
\hline Max Roll $(|b|)(\mathbf{d e g})$ & 1.352 \\
\hline Max Pitch $(|b|)(\mathbf{d e g})$ & 4.655 \\
\hline Max Yaw $(|b|)(\mathbf{d e g})$ & 0.583 \\
\hline
\end{tabular}




\section{Chapter 7}

\section{Conclusion}

The previous chapters have discussed the development of a simulation environment and a tightlycoupled PPP/INS error-state extended Kalman filter within MATLAB, the integration of the INS formulation into RTGx, and the evaluation of RTGx with flight collected data sets. This chapter summarizes the work and presents some future work.

A simulation environment was developed within MATLAB that allowed a Monte Carlo analysis of the sensitivity of PPP/INS to various conditions: such as, the intensity of multipath errors, the number of phase breaks, the satellite geometry, the atmospheric conditions, the noise characteristics of the inertial sensor, and the accuracy of GPS orbit products. The benefit of incorporating INS when confronted with scenarios that typically degrade GPS performance including poor satellite geometry and an increase level of phase breaks was characterized. The inclusion of INS is shown to offer a smoother solution leading to better absolute positioning performance in an RMS and median sense over the 100 trials. PPP/INS also exhibited the ability to initially converge quicker, which is critical for PPP. In addition, the performance increase sensitivity for including tactical grade INS when confronted with poor PPP orbit product quality and increased path dynamics was demonstrated.

After the PPP/INS formulation was verified within simulation, the INS formulation was adopted by NASA JPL's real-time positioning software RTGx. The INS formulation was then verified using flight collected data sets provided by NGA, NOAA. With the data sets, a sensitivity study was performed to see when the inclusion of INS is beneficial for position estimation. Again, it was found that INS helps significiantly when the platform is confronted with a GPS challenged environment, or poor GPS models (i.e. low fidelity troposphere model, pseudorange only solution). 


\section{Bibliography}

[1] Zumberge, J., Heflin, M., Jefferson, D., Watkins, M., and Webb, F., "Precise point positioning for the efficient and robust analysis of GPS data from large networks," Journal of Geophysical Research: Solid Earth (1978-2012), Vol. 102, No. B3, 1997, pp. 5005-5017.

[2] Dixon, K., "StarFire: A global SBAS for sub-decimeter precise point positioning," Proceedings of ION GNSS, 2006, pp. 26-29.

[3] Muellerschoen, R. and Bar-Sever, Y., "Aviation applications of NASA's global differential GPS system," Proceedings of the 2nd AIAA" Unmanned Unlimited" Conf. and Workshop 8 Exhibit, AIAA Paper, Vol. 6618, 2003.

[4] "IGS Products," .

[5] Rosen, P. A., Hensley, S., Wheeler, K., Sadowy, G., Miller, T., Shaffer, S., Muellerschoen, R., Jones, C., Zebker, H., and Madsen, S., "UAVSAR: a new NASA airborne SAR system for science and technology research," Radar, 2006 IEEE Conference on, IEEE, 2006, pp. 8-pp.

[6] Grewal, M. S., Weill, L. R., and Andrews, A. P., Global positioning systems, inertial navigation, and integration, John Wiley \& Sons, 2007.

[7] Zhang, Y. and Gao, Y., "Performance Analysis of a Tightly Coupled Kalman Filter for the Integration of un-Differenced GPS and Inertial Data," Proceedings of the 2005 National Technical Meeting of The Institute of Navigation, 2001, pp. 270-275.

[8] Du, S. and Gao, Y., "Inertial aided cycle slip detection and identification for integrated PPP GPS and INS," Sensors, Vol. 12, No. 11, 2012, pp. 14344-14362.

[9] Du, S., Integration of Precise Point Positioning and Low Cost MEMS IMU, Master's thesis, University of Calgary, November 2010. 
[10] Kjorsvik, N. S., Gjevestad, J. G. O., Broste, E., Gade, K., and Hagen, O.-K., "Tightly Coupled Precise Point Positioning and Inertial Navigation Systems," International Society for Photgrammetry and Remote Sensing European Calibration and Orientation Workshop, IPRS, 2010.

[11] Bar-Sever, Y. E., Bertiger, W. I., Dorsey, A. R., Harvey, N. E., Lu, W., Miller, K. J., Miller, M. A., Romans, L. J., Sibthorpe, A. J., Weiss, J. P., Garcia-Fernandez, M., and Gross, J., "Real-time and post-processed orbit determination and positioning, U.S. Patent No. 9,057,780 B2,” June 182015.

[12] Damiani, T., Bilich, A., and Mader, G., "Aircraft Positioning for Airborne Gravimetry: GRAVD's' Kinematic GPS Processing Challenge'," AGU Fall Meeting Abstracts, Vol. 1, 2013, p. 08.

[13] Damiani, T. M., Bilich, A., and Mader, G. L., "Evaluating Aircraft Positioning Methods for Airborne Gravimetry: First Results from GRAV-Ds Kinematic GPS Processing Challenge," .

[14] Bertiger, W., Desai, S. D., Haines, B., Harvey, N., Moore, A. W., Owen, S., and Weiss, J. P., "Single receiver phase ambiguity resolution with GPS data," Journal of Geodesy, Vol. 84, No. 5, 2010, pp. 327-337.

[15] Park, J., Helmboldt, J., Grejner-Brzezinska, D., and von Frese, R.R.B adn Wilson, T., "On Detecting Underground Nuclear Explosions with GNSS and Radio Astronomical Observations," Proceedings of The Institute of Navigation 2013 Pacific PNT Meeting, 2013, pp. 52-61.

[16] Blewitt, G., Kreemer, C., Hammond, W., Plag, H.-P., Stien, S., and Okal, E., "Rapid determination of earthquake magnitude using GPS for tsunami warning systems," Geophysical Research Letters, 2006.

[17] Dana, P. and Penrod, B., "THE ROLE OF GPS IN PRECISE TIME AND FREQUENCY DISSEMINATION," GPS World, 2010.

[18] Larson, K., Gutmann, E., Zavorotny, V., Braun, J., Williams, M., and Nievinski, F., "Can we measure snow depth with GPS receivers?" Geophysical Research Letters, Vol. 36, 2009.

[19] Misra, P. and Enge, P., Global Positioning System: Signals, Measurements and Performance Second Edition, Lincoln, MA: Ganga-Jamuna Press, 2006.

[20] Blewitt, G., Geodetic Applications of GPS, Swedish Land Survey, 1997.

[21] David, W., Broadcast Vs Precies GPS Ephemerides: A Historical Perspective, Master's thesis, Air Force Institute Of Technology, March 2002. 
[22] Roulston, A., Talbot, N., and Zhang, K., "An Evaluation of Various GPS Satellite Ephemerides," Proceedings of the Institute of Navigation Conference Global Positioning System, 2000, pp. 45-54.

[23] Wu, J., Wu, S., Hajj, G., Bertiger, W., and Lichten, S., "Effects of antenna orientation on GPS carrier phase," Manuscripta Geodaetica, 1993.

[24] "Atmospheric Correction for Troposphere and Stratosphere in Radio Ranging of Satellites," The Use of Artificial Satellites for Geodesy, Geophysics Monograph, Vol. 15, 1972.

[25] Kaplan, E. and Hegarty, C., Understanding GPS: principles and applications, Artech house, 2005 .

[26] Lagler, K., Schnidelegger, M., Bohm, J., and Nilsson, T., "GPT2: Empirical slant delay model for radio space geodetic techniques," Geophysical Research Letters, 2013.

[27] Boehm, J., Werl, B., and Schuh, H., "Troposphere mapping function for GPS and very long baseline interferometry from European Centre for Medium-Range Weather Forecasts operational analysis data," Journal of Geophysical Research, 2006.

[28] Niell, A., "Global mapping functions for the atmosphere delay at radio wavelengths," Journal of Geophysical Research, Vol. 101, 1996.

[29] Boehm, J., Niell, A., Tregoning, P., and Schuh, H., "Global Mapping Function (GMF): A new empirical mapping function based on numerical weather model data," Geophysical Research Letters, 2005.

[30] Axelrad, P., "SNR-based multipath error correction for GPS differential phase," IEEE Transactions on Aerospace and Electronic Systems, Vol. 32, 1996.

[31] Parkinson, B. and Spilker, J., Global Positioning System: Theory Applications (Volume One), Amer Inst of Aeronautics, 1996.

[32] Langely, R., "GPS Receiver System Noise," GPS World, 1997.

[33] Dawidowicz, K., "Antenna Phase Center Variations Corrections In Processing Of GPS Observations with Use of Commercial Software," Technical Sciences, Vol. 13, 2010.

[34] Watson, R., Sivaneri, V., and Gross, J., "Performance Characterization of Tightly-Coupled GNSS Precise Point Positioning Inertial Navigation within a Simulation Environment," Submitted to the 2016 AIAA Guidance Navigation and Control Conference, AIAA, 2016. 
[35] Gross J., W. R., Sivaneri V., B.-S. Y., and Bertiger W., H. B., "Integration of Inertial Navigation into Real-Time GIPSY-X (RTGx)," Institute of Navigation GNSS+, Tampa, FL, 2015.

[36] Maybeck, P. S., Stochastic models, estimation, and control, Vol. 141 of Mathematics in Science and Engineering, 1979.

[37] Woodman, O., "An introduction to inertial navigation," Tech. Rep. 696, Department of Computer Science, University of Cambridge.

[38] Jekeli, C., Inertial navigation systems with geodetic applications, Walter de Gruyter, 2001.

[39] Groves, P. D., Principles of GNSS, inertial, and multisensor integrated navigation systems, Artech House, 2013.

[40] Stevens, B. L. and Lewis, F. L., Aircraft control and simulation, John Wiley \& Sons, 2003.

[41] Cox, D., Kriegsman, B., Stonestreet, W., Kishel, J., and Calicchia, L., "Feasibility Study of GPS-Inertial Navigation for Helicopters and Study of Advanced GPS Signal Processing Techniques," 1978.

[42] Du, S. and Gao, Y., "INTEGRATION OF PPP GPS AND LOW COST IMU," Canadian Geomatics Conference 2010, 2010.

[43] "Implementation of Deeply Integrated GPS/Low-Cost IMU for Reacquisition and Tracking of Low CNR GPS Signals," National Technical Meeting of The Institute of Navigation, 2004.

[44] "A Novel Architecture for Ultra-Tight HSGPS-INS Integration," Journal of Global Positioning Systems, Vol. 7, 2008.

[45] Kalman, R. E., "A New Approach to Linear Filtering and Prediction Problems," Transactions of the ASME-Journal of Basic Engineering, Vol. 82, No. Series D, 1960, pp. 35-45.

[46] Simon, D., Optimal State Estimation: Kalman, H Infinity, and Nonlinear Approaches, Wiley, 2006.

[47] Julier, S. and Uhlmann, K., "A New Extension of the Kalman Filter to Nonlinear Systems," Proc of AeroSense: The 11th International Symposium on Aerospace/Defense Sensing, Simulation, and Controls, Orlando,Fl, 1997.

[48] Gross, J. N., Gu, Y., Rhudy, M. B., Gururajan, S., and Napolitano, M. R., "Flight-test evaluation of sensor fusion algorithms for attitude estimation," Aerospace and Electronic Systems, IEEE Transactions on, Vol. 48, No. 3, 2012, pp. 2128-2139. 
[49] GPSoft, "Satellite Navigation TOOLBOX 3.0 User's Guide," 2003.

[50] "Flight Test Evaluation of Kinematic Precise Point Positioning of Small UAVs," Internal Journal of Aerospace Engineering, 2016.

[51] Yunck, T., Bertiger, W., Wu, S., Bar-Server, Y., Christensen, E., Haines, B., Lichten, S., Muellerschoen, R., Vigue, Y., and Willis, P., "First assessment of GPS-based reduced dynamic orbit determination on TOPEX/Poseidon," Geophysical research letters, Vol. 21, No. 7, 1994, pp. $541-544$.

[52] Haines, B., Bar-Sever, Y., Bertiger, W., Desai, S., and Willis, P., "One-centimeter orbit determination for Jason-1: new GPS-based strategies," Marine Geodesy, Vol. 27, No. 1-2, 2004, pp. 299-318.

[53] Kroes, R., Montenbruck, O., Bertiger, W., and Visser, P., "Precise GRACE baseline determination using GPS," GPS Solutions, Vol. 9, No. 1, 2005, pp. 21-31.

[54] Desai, S., Bertiger, W., Gross, J., Haines, B., Harvey, N., Selle, C., Sibthorpe, A., and Weiss, J., "Results from the Reanalysis of Global GPS Data in the IGS08 Reference Frame," 2011.

[55] Bertiger, W., Bar-Sever, Y., Bokor, E., Butala, M., Dorsey, A., Gross, J., Harvey, N., Lu, W., Miller, K., Miller, M., et al., "First orbit determination performance assessment for the OCX navigation software in an operational environment," Proceedings of the 25th International Technical Meeting of The Satellite Division of the Institute of Navigation (ION GNSS+ 2012), 2012.

[56] "GNSS-Inferred Positioning System and Orbit Analysis Simulation Software," https:// gipsy-oasis.jpl.nasa.gov/, Accessed: 2016-01-25.

[57] Bierman, G., Factorization Methods for Discrete Sequential Estimation, 2006.

[58] Blewitt, G., "An automatic editing algorithm for GPS data," Geophysical Research Letters, Vol. 17, No. 3, 1990, pp. 199-202.

[59] Gross, J. N., Gu, Y., Rhudy, M., Barchesky, F. J., and Napolitano, M. R., "On-line modeling and calibration of low-cost navigation sensors," AIAA Modeling and Simulation Technologies Conference, 2011, p. 6332. 